\title{
Multiscale analysis of a prototypical model for the interaction between microstructure and surface energy
}

\author{
ANDREA BRAIDES \\ Dipartimento di Matematica, Università di Roma "Tor Vergata", \\ Via della Ricerca Scientifica, 00133 Roma, Italy \\ E-mail: braides@mat.uniroma2.it \\ CATERINA IDA ZEPPIERI \\ SISSA, Via Beirut 2, 34014 Trieste, Italy \\ E-mail: zeppieri@sissa.it
}

[Received 28 September 2007]

\begin{abstract}
The combined effect of fine heterogeneities and small gradient perturbations is analyzed by means of an asymptotic development by $\Gamma$-convergence for a family of energies related to (one-dimensional) phase transformations. We show that multi-scale effects add up to the usual sharp-interface limit, due to the homogenization of microscopic interfaces, internal and external boundary layers, optimal arrangements of microscopic oscillations, etc. Several regimes are analyzed depending on the "size" of the heterogeneity (small or large perturbations of a homogeneous situation) and their relative period as compared with the characteristic length of the phase transitions (slow or fast oscillations).
\end{abstract}

2000 Mathematics Subject Classification: 49J45, 41A60, 82B26, 74Q99.

Keywords: $\Gamma$-convergence, $\Gamma$-development, phase transitions, homogenization.

\section{Introduction}

The object of this paper is the study of the asymptotic behavior of a family of nonconvex energies of the form

$$
\int_{\Omega}\left(\mathcal{W}\left(\frac{x}{\delta}, u\right)+\varepsilon^{2}|\nabla u|^{2}\right) \mathrm{d} x
$$

(possibly with a volume constraint on $u$ ), where $\mathcal{W}$ is a periodic perturbation of a double-well potential $W$ with wells in \pm 1 , the unperturbed situation being the one studied in the seminal papers by Modica and Mortola [9, 8]. The combined descriptions obtained by homogenization with respect to the variable $\delta$ and by a sharp-interface limit as $\varepsilon \rightarrow 0$ are not sufficient in this case to fully describe the behavior of these energies at all scales, and a more complex multi-scale analysis is needed. In fact, minimal sequences tend to develop microscopic interfaces by following the oscillating minimal set of $\mathcal{W}$; such interfaces are possible in the case of slow oscillations $(\delta \gg \varepsilon)$, while are asymptotically damped in the case of fast oscillations $(\delta \ll \varepsilon)$. Note that, in contrast to [9], the latter case is the only one where minimizing sequences are strongly compact in $L^{1}$, while only weak compactness holds in the other cases. In their turn, optimal arrangements of microscopic interfaces for small perturbations of $W$ tend to oscillate between the states \pm 1 , while for large perturbations of $W$ their average tends to be 0 . As a consequence, sharp interfaces are developed only in the first case. Additional effects are due to boundary terms, to the interaction between neighboring microscopic interfaces through internal boundary layers, to the asymptotically damped 
interfaces in the case of fast oscillations, and to the optimal arrangements of microscopic interfaces in the case of large perturbations. Such a complex multi-scale analysis can be captured by looking at the development by $\Gamma$-convergence of the energies above. In this paper we focus on a prototypic one-dimensional case which we believe already contains the main features of the general case.

The prototypical energies we have in mind are governed by three different parameters: $\varepsilon>0$, the characteristic length of the phase transitions; $k \in(0,1)$, the perturbation from the phases of a given double-well potential; $\delta>0$, the period of this perturbation. More precisely, for any choice of the function $\delta=\delta(\varepsilon)$, with $\delta \rightarrow 0$ as $\varepsilon \rightarrow 0$, we consider the family of functionals

$$
F_{\varepsilon}^{k}(u)=\int_{0}^{1}\left(W^{k}\left(\frac{x}{\delta}, u\right)+\varepsilon^{2}\left(u^{\prime}\right)^{2}\right) \mathrm{d} x,
$$

where $W^{k}: \mathbb{R} \times \mathbb{R} \rightarrow[0,+\infty)$ is 1 -periodic in its first variable and on the periodicity cell $(0,1)$ is defined as

$$
W^{k}(y, s):= \begin{cases}W(s-k) & \text { if } y \in(0,1 / 2) \\ W(s+k) & \text { if } y \in(1 / 2,1)\end{cases}
$$

with $W(t)=\min \left\{(t-1)^{2},(t+1)^{2}\right\}$. A first relevant simplification to the general situation is due to this special choice of the double-well potential $W^{k}$ and to the related fact that its minimal value does not oscillate, being always equal to zero. A step in the direction of removing this simplified hypothesis is represented by a model recently proposed in [5] by Dirr, Lucia and Novaga (see also [6]). They consider a perturbation of the Modica-Mortola energy by a rapidly oscillating field with zero average; specifically,

$$
\mathcal{F}_{\varepsilon}(u)=\int_{\Omega}\left(\frac{\mathcal{W}(u)}{\varepsilon}+\varepsilon|\nabla u|^{2}+\frac{1}{\varepsilon^{\gamma}} g\left(\frac{x}{\varepsilon^{\gamma}}\right) u\right) \mathrm{d} x,
$$

where $g$ is a 1-periodic function and $\mathcal{W}$ a general double-well potential. Then for $\gamma>0$ both the amplitude and the frequency of $g$ become large (for $\varepsilon$ small) and the infimum of the energy can even tend to $-\infty$ as $\varepsilon \rightarrow 0$. Hence, in this case, to fit in the framework of $\Gamma$-convergence, the authors need to introduce an additive renormalization to the energies. In our model we do not encounter the difficulty arising from this renormalization; moreover, our particular choice permits us to detail a higher-order asymptotic analysis that is not pursued in [5].

Coming back to our model, a first observation is that for $k=0, W^{k} \equiv W$ and $[1.1$ reduces to

$$
F_{\varepsilon}(u)=\int_{0}^{1}\left(W(u)+\varepsilon^{2}\left(u^{\prime}\right)^{2}\right) \mathrm{d} x,
$$

for which a $\Gamma$-development (with respect to strong $L^{2}$-convergence) is given by the ModicaMortola Theorem [9, 8] and can be (formally) written as

$$
F_{\varepsilon}(u) \stackrel{\Gamma}{=} \int_{0}^{1} W(u) \mathrm{d} x+\varepsilon C_{W} \#(S(u))+o(\varepsilon),
$$

where $S(u)$ denotes the set of discontinuity points of $u$ and $C_{W}:=2 \int_{-1}^{1} \sqrt{W(s)} \mathrm{d} s$, with the constraint $u \in B V((0,1) ;\{ \pm 1\})$ given as understood for the second term of the development. 
Since the above $\Gamma$-development is stable under adding a volume constraint, one may prescribe the "volume" of the phases and address, for instance, the study of the minimum problems

$$
m_{\varepsilon}=\min \left\{F_{\varepsilon}(u): \int_{0}^{1} u \mathrm{~d} x=d\right\},
$$

with $|d|<1$, to exclude the trivial case of constant minimizers. Then, since the minimizers of $C_{W} \# S(u)$ are only the two functions $\pm \operatorname{sign}(x-(1-d) / 2)$, one deduces the convergence of a minimizer for $(1.3)$ to one of these functions. In addition, we have the development of minimum values

$$
m_{\varepsilon}=\varepsilon C_{W}+o(\varepsilon) \quad \text { as } \varepsilon \rightarrow 0 .
$$

Moreover, in this case it is possible to compute that the next meaningful scaling is $\varepsilon e^{-1 / 2 \varepsilon}$ and thus one may further write

$$
m_{\varepsilon}=\varepsilon C_{W}+\varepsilon e^{-1 / 2 \varepsilon} \widetilde{C}_{W}+o\left(\varepsilon e^{-1 / 2 \varepsilon}\right) \quad \text { as } \varepsilon \rightarrow 0 .
$$

However, the minimizers being essentially uniquely characterized by the analysis at order $\varepsilon$, this last information only provides a better approximation of the minimum values $m_{\varepsilon}$.

For $k>0$ we are dealing with a multi-scale energy. In this case, the spatial discontinuity of the potential makes the zero set of $W^{k}$ oscillate on a $\delta$-scale and induces the presence of microscopic oscillations whose final effect depends on the mutual vanishing rate of $\varepsilon$ and $\delta$.

As a particular case of a multi-dimensional model introduced in [7] by Francfort and Müller, we find that the energies $F_{\varepsilon}^{k} \Gamma$-converge to a homogeneous functional

$$
F_{\ell}^{k}(u)=\int_{0}^{1} W_{\ell}^{k}(u) \mathrm{d} x, \quad \text { where } \quad \ell:=\lim _{\varepsilon \rightarrow 0} \frac{\delta(\varepsilon)}{\varepsilon} .
$$

If $\ell=+\infty$, i.e., $\delta \gg \varepsilon$ (slow oscillations), the phenomenon due to periodic oscillations is dominant while the gradient perturbation turns out to be irrelevant in the limit. One has $W_{\infty}^{k} \equiv W_{\text {hom }}^{k}$, where $W_{\text {hom }}^{k}$ is given by the well-known cell formula of nonlinear scalar homogenization, which only involves the potential $W^{k}$ (see (3.2)).

If $\ell \in(0,+\infty)$, i.e., $\varepsilon \sim \delta$, oscillations and phase transitions interact and the singular perturbation contributes to the definition of the "effective potential" $W_{\ell}^{k}$ (see 3.3 ).

If $\ell=0$, i.e., $\delta \ll \varepsilon$ (fast oscillations), oscillations of $u$ on a $\delta$-scale are "forbidden" and $W_{0}^{k}$ is simply (the convex envelope of) the average of $W^{k}$ over the period (see (3.4)-3.5).

In Section 3.1 (and Section 6) we show that $F_{\ell}^{k}$ is degenerate for any choice of $k$ and $\ell$, so that a higher-order asymptotic analysis for $F_{\varepsilon}^{k}$ is needed (note that for $k \geqslant 1, F_{\ell}^{k}$ is strictly convex, which explains our choice of $k \in(0,1))$. Since we are interested in describing how the two different parameters $\varepsilon$ and $\delta$ interact in the creation of the various scales occurring in the $\Gamma$-development, we mainly focus on the two extreme regimes $\delta \gg \varepsilon$ and $\delta \ll \varepsilon$. Nonetheless, for the sake of completeness, in Section 6 we briefly discuss also the "critical" case $\delta \sim \varepsilon$.

A direct computation gives that for $\ell=+\infty, \min W_{\infty}^{k}=0=W_{\infty}^{k}(s)$ for every $s$ with $|s| \leqslant 1$, so that $F_{\infty}^{k}$ has a large set of minimizers. Then in Section 4 we turn to the analysis of the scaled energies

$$
\frac{1}{\lambda_{\infty}^{(1)}(\varepsilon)} F_{\varepsilon}^{k}
$$


with $\lambda_{\infty}^{(1)}$ a suitable positive function, vanishing as $\varepsilon \rightarrow 0$. The Modica-Mortola scale analysis performed at the micro $\delta$-level suggests that a minimizing sequence for 1.1 ) must oscillate on that scale making optimal transitions on an $\varepsilon$-layer (as, for instance, in Fig. 1).

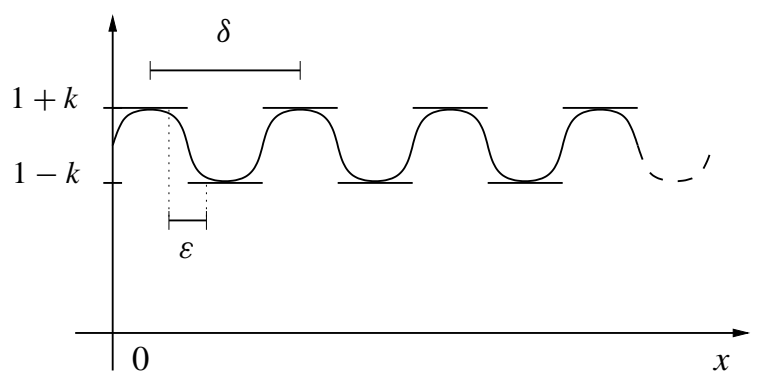

FIG. 1. Microscopic phase transitions.

In Section 4.1 we show that we may have four different types of optimal transitions characterized by three different energy contributions depending on the parameter $k$. More precisely, if these energy contributions $\varepsilon C_{i}^{k}(i=1,2,3)$ are associated to each transition as in Fig. 2 , we prove that $C_{3}^{k}>C_{1}^{k}$ and $C_{3}^{k}>C_{2}^{k}$ for every $k \in(0,1)$, while $C_{1}^{k}<C_{2}^{k}$ if and only if $k<1 / 2$ (see Remark 4.1). Hence, we have two different regimes for $k: k<1 / 2$, which we call the regime of small perturbations, and $k>1 / 2$, the regime of large perturbations.

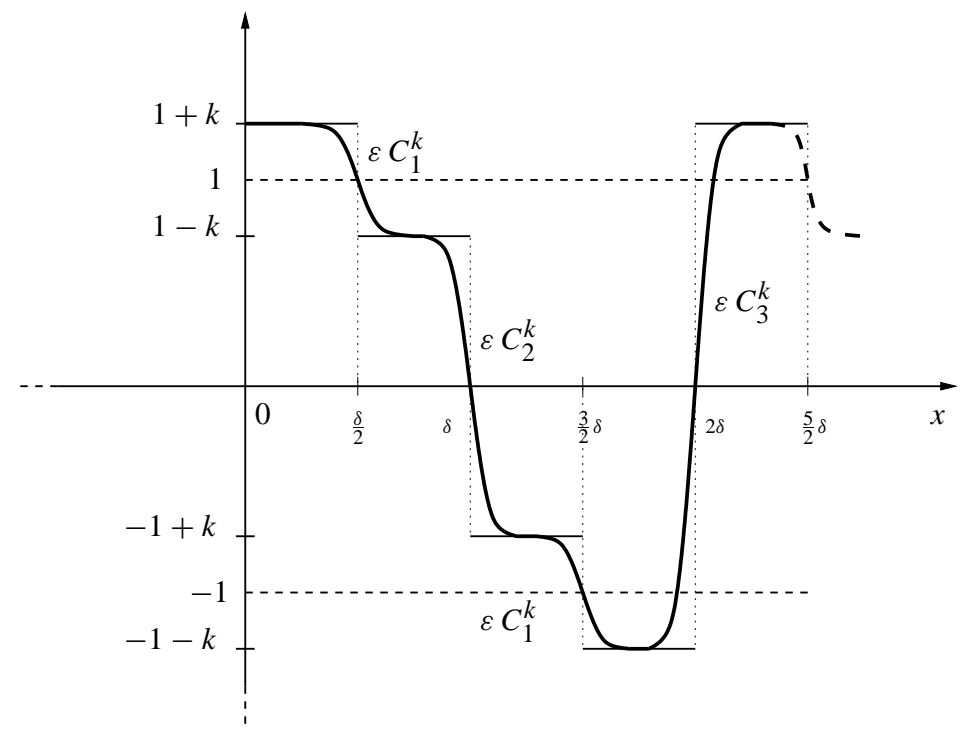

FIG. 2. Different types of optimal transitions with their energy contribution, for $k<1 / 2$.

Since each of these transitions carries an energy contribution of order $\varepsilon$, the next relevant scaling for the energy turns out to be

$$
\lambda_{\infty}^{(1)}(\varepsilon)=\varepsilon / \delta .
$$


The asymptotic behavior of (1.5) is analyzed in Theorem 4.2 which establishes that

$$
\frac{\delta}{\varepsilon} F_{\varepsilon}^{k}(u) \stackrel{\Gamma}{\longrightarrow} \int_{0}^{1} \psi^{k}(u) \mathrm{d} x=: F^{k(1)}(u)
$$

(with respect to the weak $L^{2}$-convergence) with $\psi^{k}$ as in Fig. 3 .
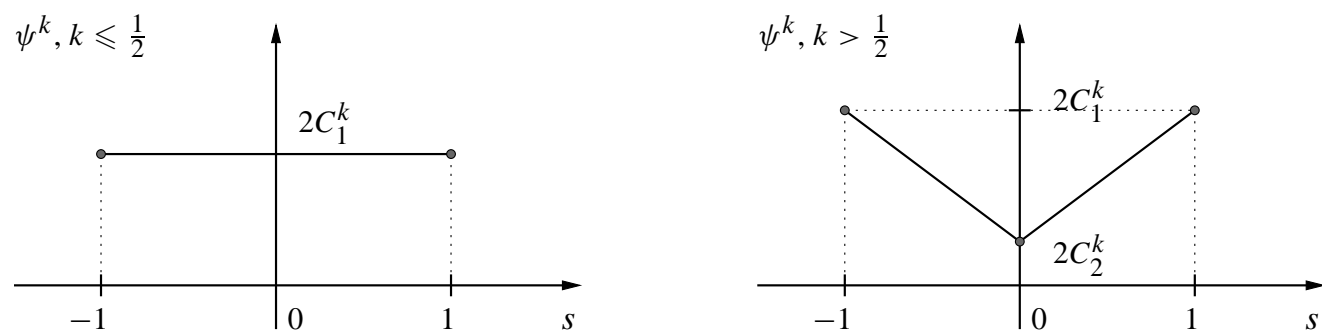

FIG. 3. The function $\psi^{k}$.

A first interesting observation is that the presence of microscopic phase transitions, with the consequent distribution of the energy of a minimizing sequence on its whole domain, leads to a $\Gamma$-limit which is again a bulk energy. This represents a difference from the Modica-Mortola result in which the energy of an optimal transition concentrates on a small layer, thus yielding a first-order energy of surface type.

From the point of view of the construction of a recovery sequence for 1.7 , Theorem 4.2 asserts that if we are in the regime of small perturbation, i.e., $k<1 / 2$, any state $|u| \leqslant 1$ can be approximated by mixing, in the right proportion, on a suitable meso-scale, minimal optimal transitions, i.e., with average 1 and average -1 . If instead we are in the case of large perturbations, i.e., $k>1 / 2$, the minimal transitions are those with average 0 which then only permit one to approximate the state $u=0$. To obtain a nonzero state we are then forced to mix minimal transitions with "expensive" ones. Moreover, (1.7) suggests that the characterization of the asymptotic behavior of sequences of minimizers, as well as the development for the minimum values, can be improved for $k<1 / 2$. In fact, for $k<1 / 2, \psi^{k} \equiv 2 C_{1}^{k}$ so that again the $\Gamma$-limit only provides the information that the weak limit of sequences of minimizers can be any function $v \in L^{2}(0,1)$ such that $|v| \leqslant 1$ a.e. Hence, in Section 4.3.1 we consider the scaled functionals

$$
\frac{F_{\varepsilon}^{k}-\frac{\varepsilon}{\delta} \min F^{k(1)}}{\lambda_{\infty}^{(2)}(\varepsilon)}=\frac{F_{\varepsilon}^{k}-\frac{\varepsilon}{\delta} \min \psi^{k}}{\lambda_{\infty}^{(2)}(\varepsilon)}=\frac{F_{\varepsilon}^{k}-\frac{\varepsilon}{\delta} 2 C_{1}^{k}}{\lambda_{\infty}^{(2)}(\varepsilon)},
$$

for a suitable scaling $\lambda_{\infty}^{(2)}(\varepsilon)$.

We observe that $F_{\varepsilon}^{k}-\frac{\varepsilon}{\delta} 2 C_{1}^{k}$ is infinitesimal on a sequence whose qualitative behavior is as in Fig. 4 .

Moreover, since a microscopic optimal transition is a solution of an optimal-profile problem stated on a $\delta / \varepsilon$-interval, on any $\delta$-period we have to consider an extra exponentially small contribution due to the interaction between two neighboring microscopic phase transitions. The total energy of a minimizing sequence for $F_{\varepsilon}^{k}-\frac{\varepsilon}{\delta} 2 C_{1}^{k}$ turns out to be of order

$$
\varepsilon+\frac{\varepsilon}{\delta} e^{-\delta / 2 \varepsilon}
$$




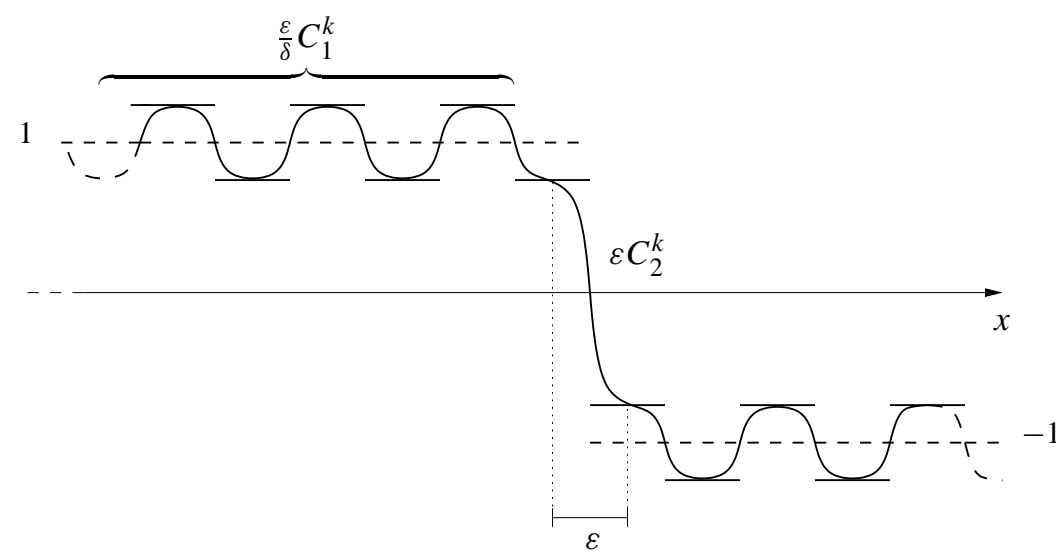

FIG. 4. A transition between the two "oscillating phases" 1 and -1 .

Then the natural assumption $\delta \gg e^{-\delta / 2 \varepsilon}$ (notice that the converse inequality would be quite restrictive for the possible choices of $\delta$ ) leads to

$$
\lambda_{\infty}^{(2)}(\varepsilon)=\varepsilon,
$$

which is the scale of the transitions between the "oscillating states" around 1 and -1 .

In terms of $\Gamma$-convergence, Lemma 4.6 and Theorem 4.7 assert that

$$
\frac{F_{\varepsilon}^{k}(u)-\frac{\varepsilon}{\delta} 2 C_{1}^{k}}{\lambda_{\infty}^{(2)}(\varepsilon)}=\frac{1}{\varepsilon} F_{\varepsilon}^{k}(u)-\frac{1}{\delta} 2 C_{1}^{k} \stackrel{\Gamma}{\longrightarrow}\left(C_{2}^{k}-C_{1}^{k}\right) \#(S(u))-C_{1}^{k}
$$

for $u \in B V((0,1) ;\{ \pm 1\})$. The extra $C_{1}^{k}$ is a boundary-layer term that would disappear under some periodicity assumptions. Hence, at scale $\varepsilon$ we get in the limit a surface energy of Modica-Mortola type but now with the convergence of minimizing sequences being only weak.

The combined computations of (1.4), (1.7) and (1.8) are formally summarized by the following development:

$$
F_{\varepsilon}^{k}(u) \stackrel{\Gamma}{=} \int_{0}^{1} W_{\mathrm{hom}}^{k}(u) \mathrm{d} x+\frac{\varepsilon}{\delta} 2 C_{1}^{k}+\varepsilon\left(\left(C_{2}^{k}-C_{1}^{k}\right) \#(S(u))-C_{1}^{k}\right)+O\left(\frac{\varepsilon}{\delta} e^{-\delta / 2 \varepsilon}\right) .
$$

We are also able to explicitly compute the optimal-profile problem energy, thus giving an accurate estimate for the error $O\left((\varepsilon / \delta) e^{-\varepsilon / 2 \delta}\right)$ (see (4.46)). A comparison between (1.2) and (1.9) shows that the interaction between periodic oscillations and phase transitions gives rise to two new scales that cannot be observed by a separate analysis of these two phenomena. These scales are $\varepsilon / \delta$, which is the scale of the microscopic interfacial energy, and $(\varepsilon / \delta) e^{-\delta / 2 \varepsilon}$, which is the scale of the interaction of microscopic interfaces.

In the case of large perturbations $k>1 / 2$ and slowly oscillating potentials $\ell=+\infty, \psi^{k}$ has the unique minimizer $s=0$ (see Fig. 3 or Fig. 5). Nonetheless, we may add an integral constraint to the problem, which in turn allows us to add an affine perturbation to the energies without changing 

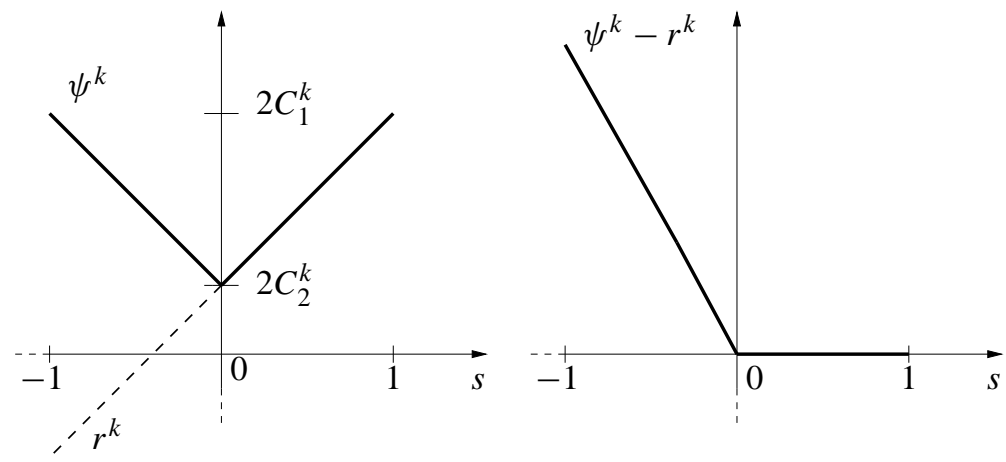

FIG. 5. The function $\psi^{k}-r^{k}$.

their minimizer. The nonstrict convexity of the function $\psi^{k}$ makes it possible to determine a $\Gamma$ development for $F_{\varepsilon}^{k}$ in this case as well. More precisely, in Section 4.3.2 we consider the functionals $(d \in(0,1)$ fixed $)$

$$
\frac{F_{\varepsilon}^{k}(u)}{\lambda_{\infty}^{(1)}}-\int_{0}^{1} r^{k}(u) \mathrm{d} x=\frac{\delta}{\varepsilon} F_{\varepsilon}^{k}(u)-\int_{0}^{1} r^{k}(u) \mathrm{d} x \text { for } u \text { such that } \int_{0}^{1} u \mathrm{~d} x=d,
$$

where $r^{k}$ is the affine perturbation chosen in such a way that $\psi^{k}(s)-r^{k}(s) \geqslant 0$ for every $|s| \leqslant 1$ and $\psi^{k}(s)-r^{k}(s)=0$ for every $0<s<1$ (see Fig. 5).

A minimizing sequence for 1.10 (or equivalently for $F_{\varepsilon}^{k}$ with the same constraint) is forced to mix minimal optimal transitions with average 0 with more expensive transitions with average 1 , thus yielding a degenerate $\Gamma$-limit having a large set of minimizers. The scale analysis for this case is quite complex and in particular highlights the presence of the new scale $\varepsilon^{2} / \delta^{2}$ which takes into account the interaction between these two different types of microscopic phase transitions (see Theorem 4.10). What happens is that for any fixed $\varepsilon>0$ a minimizer for 1.10) is the result of a suitable mixture of oscillations with average $s_{\varepsilon}>0\left(s_{\varepsilon} \rightarrow 0\right.$ as $\left.\varepsilon \rightarrow 0\right)$ and oscillations with average $1+s_{\varepsilon}$ (see Fig. 6).

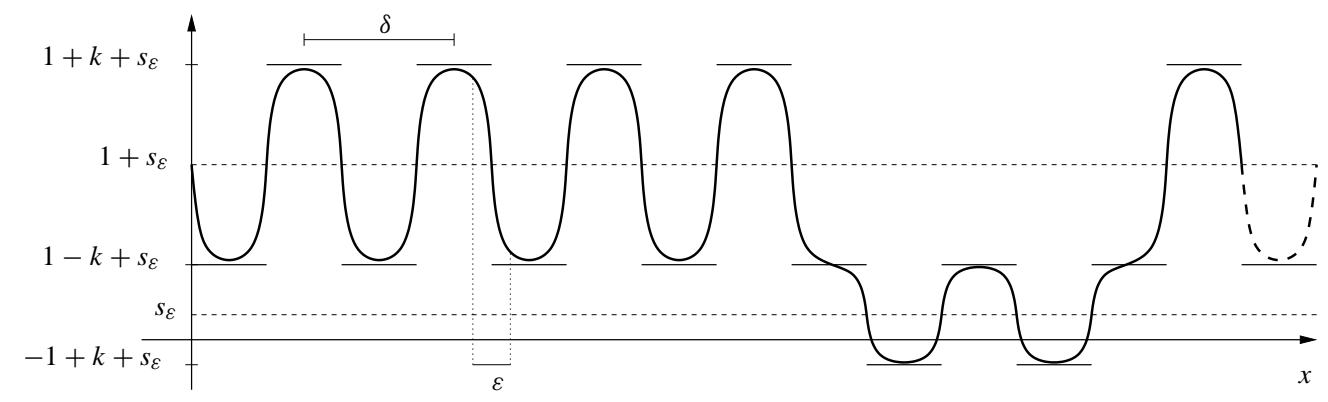

FIG. 6. A minimizing sequence in the case of large perturbations $k>1 / 2$.

Loosely speaking, using these two averages (instead of 0 and 1), a minimizer can satisfy the volume constraint using a smaller proportion of energetically expensive transitions. 
For $k \geqslant 1 / 2$, we establish the $\Gamma$-development

$$
F_{\varepsilon}^{k}(u) \stackrel{\Gamma}{=} \int_{0}^{1} W_{\text {hom }}^{k}(u) \mathrm{d} x+\frac{\varepsilon}{\delta} \int_{0}^{1} \psi^{k}(u) \mathrm{d} x-\frac{\varepsilon^{2}}{\delta^{2}}\left(C_{1}^{k}-C_{2}^{k}\right)^{2}-\varepsilon \frac{C_{1}^{k}+C_{2}^{k}}{2}+O\left(\frac{\varepsilon}{\delta} e^{-\delta / 2 \varepsilon}\right),
$$

where the term at scale $\varepsilon$ accounts now only for the effect of the external boundary layers. Moreover, the above development is valid under some additional assumption on $\delta$, needed, in this case, to get the compatibility of the $\Gamma$-limit procedure with the imposed integral constraint (see Remark 4.9. Finally, in Theorem 4.13 we carefully estimate the error $O\left((\varepsilon / \delta) e^{-\delta / 2 \varepsilon}\right)$.

For small perturbations $k<1 / 2$ and fast oscillating potentials $\ell=0$, a direct computation shows that $\min W_{0}^{k}=k^{2}=W_{0}^{k}(s)$ for every $|s| \leqslant 1$. Thus, in Section 5 we determine the scaling $\lambda_{0}^{(1)}(\varepsilon)$ in order to study the asymptotic behavior of the family of scaled functionals

$$
\frac{F_{\varepsilon}^{k}-k^{2}}{\lambda_{0}^{(1)}(\varepsilon)}
$$

We prove that, upon choosing $\delta$ sufficiently small, the presence of small scale heterogeneities does not essentially affect the $\Gamma$-convergence process at first order. Specifically, we show that even if $F_{\varepsilon}^{k}(v)-k^{2} \equiv 0$ for $v= \pm 1$ (as follows immediately from the definition of $W^{k}$ ), in this case it is more energetically convenient to oscillate "around \pm 1 " than to be identically \pm 1 , and the cost of these oscillations has a development in terms of powers of $\delta^{2} / \varepsilon^{2}$ (see Fig. 7).

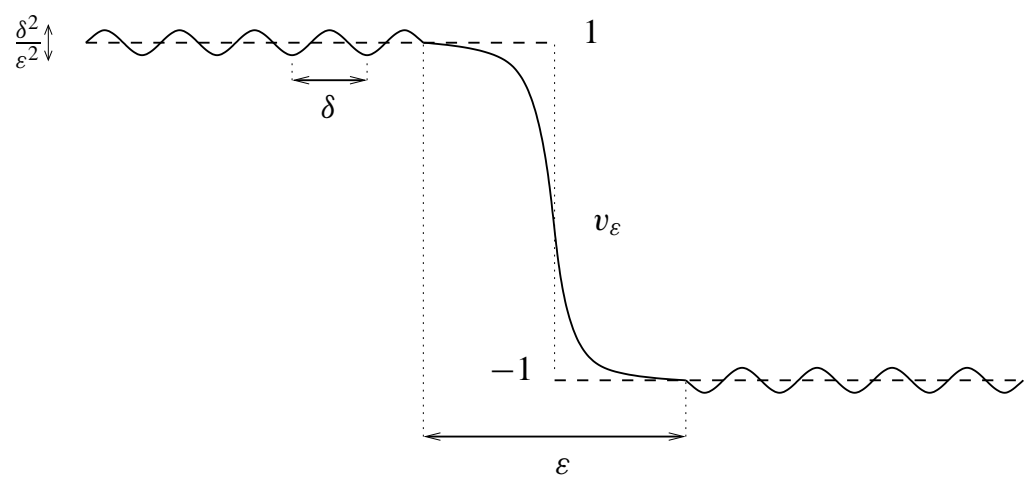

FIG. 7. The qualitative behavior of a minimizer for $F_{\varepsilon}^{k}$.

Then, since the presence of the singular gradient perturbation introduces $\varepsilon$ as the length for the layer of a transition between the two "oscillating phases" \pm 1 , we find that the contribution of a minimizing sequence for $F_{\varepsilon}^{k(0)}-k^{2}$ is of order

$$
\varepsilon+\frac{\delta^{2}}{\varepsilon^{2}}
$$

We only focus on the case $\delta \ll \varepsilon^{3 / 2}$, which yields

$$
\lambda_{0}^{(1)}(\varepsilon)=\varepsilon,
$$


since we expect to obtain constant $\Gamma$-limits for other choices of the scaling $\lambda_{0}^{(1)}(\varepsilon)$. We also notice that the asymptotic analysis for the "critical case" $\delta \simeq \varepsilon^{3 / 2}$ yields a $\Gamma$-limit of Modica-Mortola type. Nonetheless, it seems that in this case the two phenomena of oscillations and phase transition may interact in a nontrivial way, thus introducing some technical difficulties to the problem.

Under the assumption $\delta \ll \varepsilon^{3 / 2}$, in Theorem 5.1 we prove that

$$
\frac{F_{\varepsilon}^{k}(u)-k^{2}}{\lambda_{0}^{(1)}(\varepsilon)}=\frac{F_{\varepsilon}^{k}(u)-k^{2}}{\varepsilon} \stackrel{\Gamma}{\longrightarrow} C_{\left(\bar{W}^{k}-k^{2}\right)} \#(S(u))
$$

for $u \in B V((0,1) ;\{ \pm 1\})$ with

$$
\bar{W}^{k}(s):=\int_{0}^{1} W^{k}(y, s) \mathrm{d} y \quad \text { and } \quad C_{\left(\bar{W}^{k}-k^{2}\right)}:=2 \int_{-1}^{1} \sqrt{\bar{W}^{k}(s)-k^{2}} \mathrm{~d} s .
$$

In this case the equi-coercivity at scale $\varepsilon$ improves to strong- $L^{2}$ equi-coercivity, as for the ModicaMortola functional, so we may (a posteriori) compute also the "zero-order" $\Gamma$-limit with respect to strong $L^{2}$-convergence, obtaining

$$
F_{0}^{k}(u)=\int_{0}^{1} \bar{W}^{k}(u) \mathrm{d} x .
$$

Thus, for $\delta \ll \varepsilon^{3 / 2}$ and $k<1 / 2$ we find that a $\Gamma$-development for $F_{\varepsilon}^{k}$ (with respect to strong $L^{2}$-convergence) is given by

$$
F_{\varepsilon}^{k}(u) \stackrel{\Gamma}{=} \int_{0}^{1} \bar{W}^{k}(u) \mathrm{d} x+\varepsilon C_{\left(\bar{W}^{k}-k^{2}\right)} \#(S(u))+O\left(\frac{\delta^{2}}{\varepsilon^{2}}\right) .
$$

The above development in particular shows that in this case we may formally first perform the homogenization procedure for fixed $\varepsilon$ by letting $\delta \rightarrow 0$, and then apply the Modica-Mortola Theorem to

$$
\int_{0}^{1}\left(\bar{W}^{k}(u)-k^{2}+\varepsilon^{2}\left(u^{\prime}\right)^{2}\right) \mathrm{d} x .
$$

Theorem 5.3 states that in this case the scale analysis performed for $k<1 / 2$ applies unchanged for $k \geqslant 1 / 2$, thus yielding an analogous $\Gamma$-convergence result.

Finally, the case of potentials oscillating on the phase transition characteristic length scale, i.e., the case $\delta \sim \varepsilon$, can also be described by a development, taking into account that optimal sequences still have the form described in Fig. 7, with finite oscillations around \pm 1 (or around two explicitly computed positive values for the case of large perturbations with a positive volume constraint; see (6.1), now neither following the zeroes of $W^{k}$ as for slow oscillations, nor asymptotically dumped as for fast oscillations. This case is briefly described in the last section.

\section{Development by $\Gamma$-convergence}

For the reader's convenience and in order to fix notation, in this section we recall the notion of development by $\Gamma$-convergence (or in brief, $\Gamma$-development) and the related terminology. For an introduction to the subject, we refer the reader to the classical paper by Anzellotti and Baldo [2] and to the more recent work by Braides and Truskinovsky [4]. 
The procedure leading to a development by $\Gamma$-convergence is aimed at a better characterization of the asymptotic behavior of a family of minimum problems when the $\Gamma$-limit is degenerate. Specifically, if $\left(F_{\varepsilon}\right)$ is a given family of equi-coercive microscopic energies, the fundamental property of $\Gamma$-convergence can be sketched as

$$
F_{\varepsilon} \stackrel{\Gamma}{\longrightarrow} F^{(0)} \Rightarrow\left\{\begin{array}{l}
m_{\varepsilon}:=\min F_{\varepsilon} \rightarrow m^{(0)}:=\min F^{(0)} \\
\left\{\text { limits of minimizers of } F_{\varepsilon}\right\} \subseteq \operatorname{argmin}\left(F^{(0)}\right),
\end{array}\right.
$$

where $\operatorname{argmin}\left(F^{(0)}\right):=\left\{u: F^{(0)}(u)=m^{(0)}\right\}$ and the inclusion may be proper. Hence, in general the description given by $F^{(0)}$ may fail to completely characterize the asymptotic behavior of the family $\left(F_{\varepsilon}\right)$. Then the idea is that the computation of the $\Gamma$-limit $F^{(0)}$, the zero-order $\Gamma$-limit, is only the first step in the description of the asymptotic behavior of $F_{\varepsilon}$, as it may be necessary to refine the above limit procedure to select those minimizers of $F^{(0)}$ which are actually limits of minimizing sequences of $F_{\varepsilon}$.

The most intuitive refinement procedure of standard $\Gamma$-convergence is the iteration of the successive $\Gamma$-limits. Once a $\Gamma$-limit $F^{(0)}$ of a family $\left(F_{\varepsilon}\right)$ is computed, and the next meaningful scale $\lambda^{(1)}(\varepsilon)$ (by a scale we mean a function $\lambda^{(1)}(\varepsilon)>0$ such that $\lambda^{(1)}(\varepsilon) \rightarrow 0$ as $\varepsilon \rightarrow 0$ ) is conjectured, we may look at the $\Gamma$-limit of the scaled family of energies

$$
F_{\varepsilon}^{(1)}(u):=\frac{F_{\varepsilon}(u)-m^{(0)}}{\lambda^{(1)}(\varepsilon)}
$$

and, if it exists, we denote it by $F^{(1)}$, the first-order $\Gamma$-limit (or, if more meaningful, the $\Gamma$-limit at order $\lambda^{(1)}(\varepsilon)$ ). Notice that the domain of $F^{(1)}$ is, by definition, a subset of the set of minimum points of $F^{(0)}$, i.e., $\operatorname{dom}\left(F^{(1)}\right) \subseteq \operatorname{argmin}\left(F^{(0)}\right)$. If $F^{(1)}$ is not trivial (which is the case if the scale $\lambda^{(1)}(\varepsilon)$ is "meaningful"), then the iterated application of the property of convergence of minima for $\Gamma$-converging sequences leads to a development of the minimum values

$$
m_{\varepsilon}=m^{(0)}+\lambda^{(1)}(\varepsilon) m^{(1)}+o\left(\lambda^{(1)}(\varepsilon)\right) \quad \text { as } \varepsilon \rightarrow 0,
$$

where $m^{(1)}:=\min F^{(1)}$. It is also clear that the minimizers for $F_{\varepsilon}^{(1)}$ are exactly those for $F_{\varepsilon}$; then we deduce that the limit of minimizers not only minimizes $F^{(0)}$ but also $F^{(1)}$. Loosely speaking, we have

$$
\left\{\text { limits of minimizers of } F_{\varepsilon}\right\} \subseteq \operatorname{argmin}\left(F^{(1)}\right) \subseteq \operatorname{argmin}\left(F^{(0)}\right),
$$

thus we have actually made a selection from minimum points of $F^{(0)}$. The combined computation of the zero-order and first-order $\Gamma$-limit as above is formally written as the $\Gamma$-development

$$
F_{\varepsilon} \stackrel{\Gamma}{=} F^{(0)}+\lambda^{(1)}(\varepsilon) F^{(1)}+o\left(\lambda^{(1)}(\varepsilon)\right),
$$

with $o\left(\lambda^{(1)}(\varepsilon)\right)$ meaning that the next relevant scale is of higher order than $\lambda^{(1)}(\varepsilon)$ as $\varepsilon \rightarrow 0$.

If necessary, this procedure can be iterated to obtain other scales $\lambda^{(2)}(\varepsilon), \lambda^{(3)}(\varepsilon)$, etc., and consequently other terms in the development. This may provide a considerable improvement of the description of the minimizing sequences, and in some cases may give a complete characterization of the asymptotic behavior of $\left(F_{\varepsilon}\right)$. Moreover, we remark that since in the applications one would like to construct "theories" operative at small but finite $\varepsilon$, a development by $\Gamma$-convergence can also be viewed as the simplest way to bring a small scale back into the problem (see [4]). 


\section{Zero-order $\Gamma$-limit}

In what follows, our notation will be consistent with that introduced in Section 2 .

Our energy is a particular one-dimensional version of a more general multi-dimensional energy introduced by Francfort and Müller [7]. Thus, having in mind the idea of a $\Gamma$-development for (1.1), in this section we adapt to our setting the $\Gamma$-convergence results of Theorems 2.1 and 2.3 in [7]. Those two results are summarized in the following theorem.

THEOREM 3.1 Let $\delta=\delta(\varepsilon)>0$ be such that $\delta \rightarrow 0$ as $\varepsilon \rightarrow 0$ and set

$$
\ell:=\lim _{\varepsilon \rightarrow 0} \frac{\delta(\varepsilon)}{\varepsilon} .
$$

Then the family of functionals $F_{\varepsilon}^{k}: L^{2}(0,1) \rightarrow(0,+\infty]$ defined by

$$
F_{\varepsilon}^{k}(u)= \begin{cases}\int_{0}^{1}\left(W^{k}\left(\frac{x}{\delta}, u\right)+\varepsilon^{2}\left(u^{\prime}\right)^{2}\right) \mathrm{d} x & \text { if } u \in W^{1,2}(0,1) \\ +\infty & \text { otherwise }\end{cases}
$$

$\Gamma$-converges with respect to weak $L^{2}$-convergence to the homogeneous functional defined on $L^{2}(0,1)$ by

$$
F_{\ell}^{k(0)}(u)=\int_{0}^{1} W_{\ell}^{k}(u) \mathrm{d} x .
$$

The integrand $W_{\ell}^{k}$ depends on $\ell$ in the following way:

(1) if $\ell=+\infty$, then

$$
W_{\infty}^{k}(s)=\inf \left\{\int_{0}^{1} W^{k}(x, v) \mathrm{d} x: v \in L^{2}(0,1), \int_{0}^{1} v \mathrm{~d} x=s\right\}
$$

(2) if $\ell \in(0,+\infty)$, then

$$
W_{\ell}^{k}(s)=\inf _{n \in \mathbb{N}} \inf \left\{f_{0}^{n}\left(W^{k}(x, v)+\frac{1}{\ell^{2}}\left(v^{\prime}\right)^{2}\right) \mathrm{d} x: v \in W^{1,2}(0, n), f_{0}^{n} v \mathrm{~d} x=s\right\}
$$

(3) if $\ell=0$, then

$$
W_{0}^{k}(s)=\left(\bar{W}^{k}\right)^{* *}(s),
$$

where

$$
\bar{W}^{k}(s)=\int_{0}^{1} W^{k}(y, s) \mathrm{d} y
$$

and $\left(\bar{W}^{k}\right)^{* *}$ is the convex envelope of $\bar{W}^{k}$.

REMARK 3.2 From the definition of $W^{k}$, a priori we only know that the family $\left(F_{\varepsilon}^{k}\right)$ is equicoercive with respect to weak $L^{2}$-convergence (for any choice of $\delta=\delta(\varepsilon)$ ); for this reason, in Theorem 3.1, the $\Gamma$-limit is computed, in each regime, with respect to that convergence. 


\subsection{The effective potential $W_{\ell}^{k}$}

The starting point of our analysis is a complete characterization of the zero-order $\Gamma$-limit $F_{\ell}^{k(0)}$. Then, recalling the definition of our given $W^{k}$, in this section we compute the explicit expression for the effective potential $W_{\ell}^{k}$. Here we only treat the cases $\ell=+\infty$ and $\ell=0$; since the case $\ell \in(0,+\infty)$ needs a different investigation involving some of the techniques developed in the next sections, we refer the reader to Section 6 for that case.

If $\ell=+\infty$, Theorem 3.1 asserts that $W_{\infty}^{k}$ is given in terms of the cell formula 3.2, which is equivalent to

$$
W_{\infty}^{k}(s)=\min \left\{\int_{0}^{1}\left(W^{k}\right)^{* *}(x, v) \mathrm{d} x: v \in L^{2}(0,1), \int_{0}^{1} v \mathrm{~d} x=s\right\},
$$

where the convexification is taken with respect to the second variable. Thus by using Jensen's inequality it is easy to check that

$$
W_{\infty}^{k}(s)=\min \left\{\frac{1}{2} W^{* *}\left(s_{1}-k\right)+\frac{1}{2} W^{* *}\left(s_{2}+k\right): s_{1}+s_{2}=2 s\right\} .
$$

Finally, a straightforward calculation gives

$$
W_{\infty}^{k}(s)=W^{* *}(s)= \begin{cases}0 & \text { if }|s| \leqslant 1 \\ (|s|-1)^{2} & \text { if }|s|>1\end{cases}
$$

If $\ell=0$, then trivially

$$
\bar{W}^{k}(s)=\frac{1}{2}\left(W^{k}(s-k)+W^{k}(s+k)\right)= \begin{cases}s^{2}+(1-k)^{2} & \text { if }|s| \leqslant k \\ (|s|-1)^{2}+k^{2} & \text { if }|s|>k\end{cases}
$$

hence by a direct computation we get

$$
W_{0}^{k}(s)= \begin{cases}k^{2} & \text { if }|s| \leqslant 1 \\ (|s|-1)^{2}+k^{2} & \text { if }|s|>1\end{cases}
$$

for $k \leqslant 1 / 2$, while

$$
W_{0}^{k}(s)= \begin{cases}s^{2}+(1-k)^{2} & \text { if }|s| \leqslant k-1 / 2, \\ (2 k-1)|s|-k+3 / 4 & \text { if } k-1 / 2<|s|<k+1 / 2, \\ (|s|-1)^{2}+k^{2} & \text { if }|s|>k+1 / 2,\end{cases}
$$

for $k>1 / 2$.

Now, the effective potential $W_{\ell}^{k}$ has a large set of minimizers for both $\ell=+\infty$ and $\ell=0$, $k \leqslant 1 / 2$; more precisely, $W_{\ell}^{k}(s)=\min W_{\ell}^{k}$ for every $s$ such that $|s| \leqslant 1$. At this point some scale analysis must be performed for both $\ell=+\infty$ and $\ell=0, k \leqslant 1 / 2$, to understand what the next relevant scaling is. We expect this scaling to depend on the regime $\ell$ and on the parameter $k$ as well but, not to overburden notation, we only indicate the dependence on $\ell$ so that in what follows we denote the first meaningful scaling by $\lambda_{\ell}^{(1)}(\varepsilon)$. 

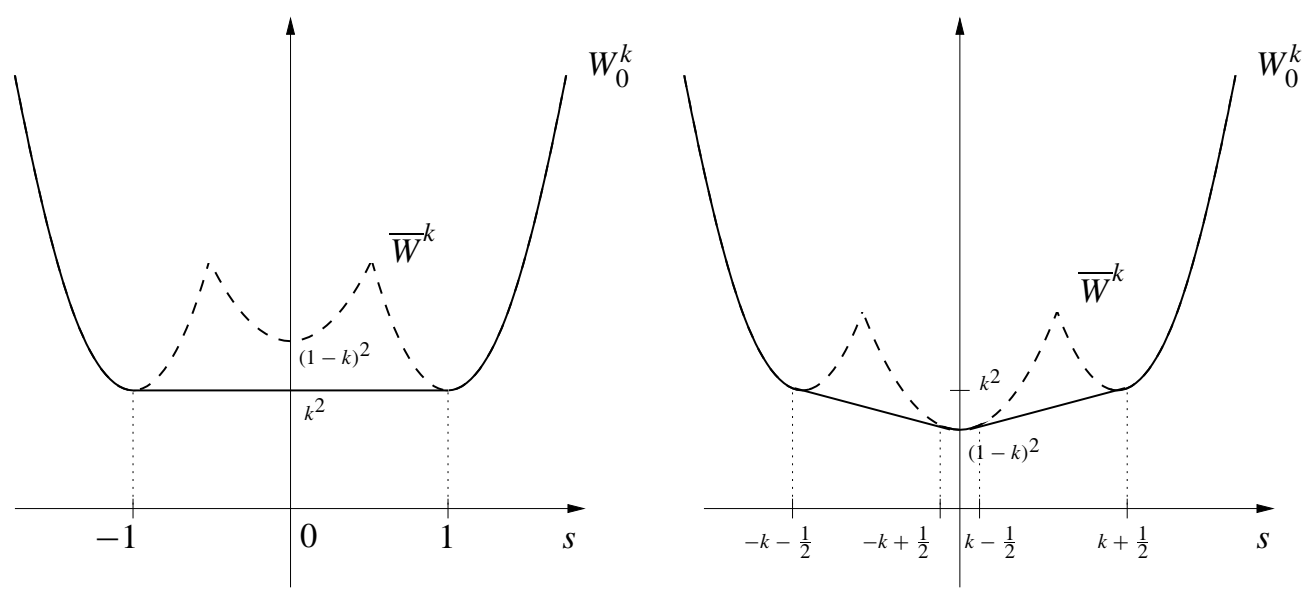

FIG. 8. The effective potential $W_{0}^{k}$ for $k<1 / 2$ and $k>1 / 2$.

Finally, as for the remaining case $\ell=0, k>1 / 2$, we want to point out that the nonstrict convexity of $W_{0}^{k}$ (see Fig. 8 permits us to determine an asymptotic development for $F_{\varepsilon}^{k}$ in this case too by adding an integral constraint to the problem, which in turn allows one to add an affine perturbation to the energies without changing their minimizers (see Section 5, and also Section 4.3.2.

\section{4. $\delta \gg \varepsilon$ : the case of slow oscillations}

In this section we treat the case when the scale of oscillation $\delta$ is much larger than the scale of the transition layer $\varepsilon$, i.e., the case $\ell=+\infty$.

In order to guess what the first meaningful scale $\lambda_{\infty}^{(1)}(\varepsilon)$ is, we start by performing a preliminary qualitative scale analysis. Using the same argument proposed to examine the Modica-Mortola model [8, 9] we estimate the order of $m_{\varepsilon}^{k}:=\min F_{\varepsilon}^{k}$ as $\varepsilon \rightarrow 0$. To this end, we focus on a single $\delta$-interval; to fix ideas, say the interval $(0, \delta)$. Now, when we come to minimize $F_{\varepsilon}^{k}$, on one hand the term $\int_{0}^{\delta} W^{k}(x / \delta, u) \mathrm{d} x$ favors those configurations which take values close to the (varying) zero set of $W^{k}$, i.e., close to (at least) two different constant values: one chosen in $\{1+k,-1+k\}$ when $x \in(0, \delta / 2)$, and the other chosen in $\{1-k,-1-k\}$ when $x \in(\delta / 2, \delta)$. In other words, the potential term in the energy favors a phenomenon of phase separation. On the other hand, the gradient term $\varepsilon^{2} \int_{0}^{\delta}\left(u^{\prime}\right)^{2} \mathrm{~d} x$ penalizes spatial inhomogeneities, thus inducing a phase transition phenomenon as well. When $\varepsilon$ is small the first term prevails, and the minimum of

$$
\int_{0}^{\delta}\left(W^{k}\left(\frac{x}{\delta}, u\right)+\varepsilon^{2}\left(u^{\prime}\right)^{2}\right) \mathrm{d} x
$$

is attained at a function which takes "mainly" values close to the set $\{1+k,-1+k\}$ in $(0, \delta / 2)$ and close to $\{1-k,-1-k\}$ in $(\delta / 2, \delta)$, but which also makes a transition on a "thin" layer around $\delta / 2$. Then a scaling argument (see e.g. [1] and [3, Chapter 6]) proves that the transition between two different zeroes chosen as above actually occurs in a layer of thickness of order $\varepsilon$ (recall that 
$\delta \gg \varepsilon$ ) and gives an energy contribution of order $\varepsilon$. Clearly the previous heuristics can be repeated on each $\delta$-interval, thus yielding a total energy contribution of order $\varepsilon / \delta$. Hence, we claim that $\lambda_{\infty}^{(1)}(\varepsilon)=\varepsilon / \delta$, and the proof of this claim will be made rigorous with Theorem 4.2

\subsection{Estimate for the phase-transition energy}

We now move the first step towards a rigorous justification of the qualitative argument discussed in the previous section. In what follows, we make use of some well-known facts relating to the so-called optimal-profile problem in the Modica-Mortola model. For a detailed treatment of the one-dimensional case, we refer the reader to [1, Section 3a] or [3, Remark 6.1].

We want to find an explicit formula for the phase-transition energy; for this purpose we set

$$
W_{1}^{k}(s):=W(s-k), \quad W_{2}^{k}(s):=W(s+k),
$$

and for any fixed $\varepsilon>0$, we let $x_{1}, x_{2} \in \mathbb{R}$ be such that $x_{1}<x_{2}, x_{2}-x_{1} \leqslant \delta / 2$ and $\delta / 2 \in\left(x_{1}, x_{2}\right)$. We start by estimating the contribution of the integration on $\left(x_{1}, x_{2}\right)$ in $F_{\varepsilon}^{k}(u)$ in terms of $z_{1}:=$ $u\left(x_{1}\right)$ and $z_{2}:=u\left(x_{2}\right)$. After setting $v(x):=u(\varepsilon x)$, we have

$$
\begin{aligned}
\int_{x_{1}}^{x_{2}}\left(W^{k}\left(\frac{x}{\delta}, u\right)\right. & \left.+\varepsilon^{2}\left(u^{\prime}\right)^{2}\right) \mathrm{d} x \\
& =\varepsilon\left(\int_{x_{1}}^{\delta / 2}\left(\frac{1}{\varepsilon} W_{1}^{k}(u)+\varepsilon\left(u^{\prime}\right)^{2}\right) \mathrm{d} x+\int_{\delta / 2}^{x_{2}}\left(\frac{1}{\varepsilon} W_{2}^{k}(u)+\varepsilon\left(u^{\prime}\right)^{2}\right) \mathrm{d} x\right) \\
& =\varepsilon\left(\int_{x_{1} / \varepsilon}^{\delta / 2 \varepsilon}\left(W_{1}^{k}(v)+\left(v^{\prime}\right)^{2}\right) \mathrm{d} x+\int_{\delta / 2 \varepsilon}^{x_{2} / \varepsilon}\left(W_{2}^{k}(v)+\left(v^{\prime}\right)^{2}\right) \mathrm{d} x\right) .
\end{aligned}
$$

By the change of variable $y=x-\delta / 2 \varepsilon$, (4.1) becomes

$$
\varepsilon\left(\int_{-T_{1}}^{0}\left(W_{1}^{k}(z)+\left(z^{\prime}\right)^{2}\right) \mathrm{d} y+\int_{0}^{T_{2}}\left(W_{2}^{k}(z)+\left(z^{\prime}\right)^{2}\right) \mathrm{d} y\right),
$$

with $T_{1}:=\left(\delta-2 x_{1}\right) / 2 \varepsilon, T_{2}:=\left(2 x_{2}-\delta\right) / 2 \varepsilon$ and $z(y):=v(y+\delta / 2 \varepsilon)$. Hence, a lower bound for the energy of a transition between the values $z_{1}, z_{2}$ is given by

$$
\begin{aligned}
\varepsilon \inf _{T_{1}, T_{2}>0} \inf \left\{\int_{-T_{1}}^{0}\left(W_{1}^{k}(z)+\left(z^{\prime}\right)^{2}\right) \mathrm{d} y\right. & +\int_{0}^{T_{2}}\left(W_{2}^{k}(z)+\left(z^{\prime}\right)^{2}\right) \mathrm{d} y: \\
& \left.z \in W^{1,2}\left(-T_{1}, T_{2}\right), z\left(-T_{1}\right)=z_{1}, z\left(T_{2}\right)=z_{2}\right\} .
\end{aligned}
$$

Now let $Z_{i}^{k}$ be the set of zeroes of $W_{i}^{k}$ for $i=1$, 2, i.e.,

$$
Z_{1}^{k}=\{-1+k, 1+k\}, \quad Z_{2}^{k}=\{-1-k,-1+k\} ;
$$

if $z_{i} \in Z_{i}^{k}(i=1,2)$ we know that

$$
\begin{aligned}
\inf _{T_{1}>0} \inf & \left\{\int_{-T_{1}}^{0}\left(W_{1}^{k}(z)+\left(z^{\prime}\right)^{2}\right) \mathrm{d} y: z \in W^{1,2}\left(-T_{1}, 0\right), z\left(-T_{1}\right)=z_{1}, z(0)=z_{0}\right\} \\
& =\inf \left\{\int_{-\infty}^{0}\left(W_{1}^{k}(z)+\left(z^{\prime}\right)^{2}\right) \mathrm{d} y: z \in W_{\mathrm{loc}}^{1,2}(-\infty, 0), z(-\infty)=z_{1}, z(0)=z_{0}\right\}
\end{aligned}
$$


and

$$
\begin{aligned}
\inf _{T_{2}>0} & \inf \left\{\int_{0}^{T_{2}}\left(W_{2}^{k}(z)+\left(z^{\prime}\right)^{2}\right) \mathrm{d} y: z \in W^{1,2}\left(0, T_{2}\right), z(0)=z_{0}, z\left(T_{2}\right)=z_{2}\right\} \\
& =\inf \left\{\int_{0}^{+\infty}\left(W_{2}^{k}(z)+\left(z^{\prime}\right)^{2}\right) \mathrm{d} y: z \in W_{\mathrm{loc}}^{1,2}(0,+\infty), z(0)=z_{0}, z(+\infty)=z_{2}\right\}
\end{aligned}
$$

where $z(-\infty)$ and $z(+\infty)$ are understood as the existence of the corresponding limits. Then it is easy to check that 4.2 can be rewritten in terms of the two optimal profile problems 4.3 , and 4.4 , as

$$
\begin{aligned}
\varepsilon \inf _{z_{0}}\left\{\inf \left\{\int_{-\infty}^{0}\left(W_{1}^{k}(z)+\left(z^{\prime}\right)^{2}\right) \mathrm{d} y: z \in W_{\mathrm{loc}}^{1,2}(-\infty, 0), z(-\infty)=z_{1}, z(0)=z_{0}\right\}\right. \\
\left.\quad+\inf \left\{\int_{0}^{+\infty}\left(W_{2}^{k}(z)+\left(z^{\prime}\right)^{2}\right) \mathrm{d} y: z \in W_{\mathrm{loc}}^{1,2}(0,+\infty), z(0)=z_{0}, z(+\infty)=z_{2}\right\}\right\},
\end{aligned}
$$

and finally as

$$
\varepsilon \inf _{z_{0}}\left\{2\left|\int_{z_{1}}^{z_{0}} \sqrt{W_{1}^{k}(s)} \mathrm{d} s\right|+2\left|\int_{z_{0}}^{z_{2}} \sqrt{W_{2}^{k}(s)} \mathrm{d} s\right|\right\} .
$$

Hence, if for every $\zeta_{1}, \zeta_{2} \in \mathbb{R}$, we set

$$
C_{W^{k}}\left(\zeta_{1}, \zeta_{2}\right):=\inf _{z_{0}}\left\{2\left|\int_{\zeta_{1}}^{z_{0}} \sqrt{W_{1}^{k}(s)} \mathrm{d} s\right|+2\left|\int_{z_{0}}^{\zeta_{2}} \sqrt{W_{2}^{k}(s)} \mathrm{d} s\right|\right\},
$$

we have

$$
\int_{x_{1}}^{x_{2}}\left(W^{k}\left(\frac{x}{\delta}, u\right)+\varepsilon^{2}\left(u^{\prime}\right)^{2}\right) \mathrm{d} x \geqslant \varepsilon C_{W^{k}}\left(z_{1}, z_{2}\right) .
$$

Finally, recalling the definition of the potential $W^{k}$, in order to explicitly compute $C_{W^{k}}\left(z_{1}, z_{2}\right)$ we have to distinguish three cases.

Case 1: $z_{1}=1+k, z_{2}=1-k$.

$$
\begin{aligned}
C_{1}^{k}:=C_{W^{k}}(1+k, 1-k) & =\inf _{z_{0}}\left\{2 \int_{z_{0}}^{1+k} \sqrt{W_{1}^{k}(s)} \mathrm{d} s+2 \int_{1-k}^{z_{0}} \sqrt{W_{2}^{k}(s)} \mathrm{d} s\right\} \\
& =2 \int_{1}^{1+k} \sqrt{W_{1}^{k}(s)} \mathrm{d} s+2 \int_{1-k}^{1} \sqrt{W_{2}^{k}(s)} \mathrm{d} s=2 k^{2} .
\end{aligned}
$$

Moreover, it is immediate that $C_{W^{k}}(-1+k,-1-k)=C_{1}^{k}$.

Case 2: $z_{1}=-1+k, z_{2}=1-k$.

$$
\begin{aligned}
C_{2}^{k}:=C_{W^{k}}(-1+k, 1-k) & =\inf _{z_{0}}\left\{2 \int_{-1+k}^{z_{0}} \sqrt{W_{1}^{k}(s)} \mathrm{d} s+2 \int_{z_{0}}^{1-k} \sqrt{W_{2}^{k}(s)} \mathrm{d} s\right\} \\
& =2 \int_{-1+k}^{0} \sqrt{W_{1}^{k}(s)} \mathrm{d} s+2 \int_{0}^{1-k} \sqrt{W_{2}^{k}(s)} \mathrm{d} s=2(1-k)^{2} .
\end{aligned}
$$


Case 3: $z_{1}=1+k, z_{2}=-1-k$.

$$
\begin{aligned}
C_{3}^{k}:=C_{W^{k}}(1+k,-1-k) & =\inf _{z_{0}}\left\{2 \int_{z_{0}}^{k+1} \sqrt{W_{1}^{k}(s)} \mathrm{d} s+2 \int_{-1-k}^{z_{0}} \sqrt{W_{2}^{k}(s)} \mathrm{d} s\right\} \\
& =2 \int_{1}^{k+1} \sqrt{W_{1}^{k}(s)} \mathrm{d} s+2 \int_{-k-1}^{1} \sqrt{W_{2}^{k}(s)} \mathrm{d} s=2\left(1+k^{2}\right) .
\end{aligned}
$$

Remark 4.1 The constant $C_{3}^{k}$ is greater than both $C_{1}^{k}$ and $C_{2}^{k}$ for every $k \in(0,1)$, i.e., the transition between the two extreme zeroes $1+k$ and $-1-k$ is always energetically unfavorable, while

$$
C_{1}^{k}<C_{2}^{k} \Leftrightarrow k<1 / 2,
$$

or in other words, the transition from $1+k$ to $1-k$ (or equivalently from $-1+k$ to $-1-k$ ) is more convenient than the one from $-1+k$ to $1-k$ if and only if $k<1 / 2$.

\subsection{First-order $\Gamma$-limit}

We are now ready to state the $\Gamma$-convergence result for the family of scaled functionals

$F_{\varepsilon}^{k(1)}(u):=\frac{F_{\varepsilon}^{k}(u)}{\lambda_{\infty}^{(1)}(\varepsilon)}=\frac{\delta}{\varepsilon} F_{\varepsilon}^{k}(u)= \begin{cases}\int_{0}^{1}\left(\frac{\delta}{\varepsilon} W^{k}\left(\frac{x}{\delta}, u\right)+\varepsilon \delta\left(u^{\prime}\right)^{2}\right) \mathrm{d} x & \text { if } u \in W^{1,2}(0,1), \\ +\infty & \text { otherwise. }\end{cases}$

Note that to ease notation, in $F_{\varepsilon}^{k(1)}$ we omit the dependence on $\ell$.

THEOREM 4.2 The family of functionals $F_{\varepsilon}^{k(1)}$ defined in $4.9 \Gamma$-converges with respect to weak $L^{2}$-convergence to the integral functional defined on $L^{2}(0,1)$ by

$$
F^{k(1)}(u)= \begin{cases}\int_{0}^{1} \psi^{k}(u) \mathrm{d} x & \text { if } u \in L^{2}(0,1) \text { and }|u| \leqslant 1 \text { a.e. } \\ +\infty & \text { otherwise }\end{cases}
$$

where

$$
\psi^{k}(s)= \begin{cases}2 C_{1}^{k} & \text { if } k \leqslant 1 / 2 \\ 2\left(C_{1}^{k}-C_{2}^{k}\right)|s|+2 C_{2}^{k} & \text { if } k>1 / 2\end{cases}
$$

Before proving this we need some preliminary results.

In the following proposition, $\eta$ is the small positive parameter that we will let go to zero in the $\Gamma$-limit procedure.

Proposition 4.3 (i) The family of functionals $G_{\eta}^{k}$ defined on $L^{2}(-1 / 4,1 / 4)$ by

$$
G_{\eta}^{k}(u)= \begin{cases}\int_{-1 / 4}^{1 / 4}\left(\frac{1}{\eta} W^{k}(x, u)+\eta\left(u^{\prime}\right)^{2}\right) \mathrm{d} x & \text { if } u \in W^{1,2}(-1 / 4,1 / 4), \\ +\infty & \text { otherwise }\end{cases}
$$


$\Gamma$-converges with respect to strong $L^{2}$-convergence to the functional defined on $L^{2}(-1 / 4,1 / 4)$ by

$$
G^{k}(u)=\left\{\begin{aligned}
& C_{W}(\#(S(u))-1)+C_{W^{k}}\left(u\left(0^{+}\right), u\left(0^{-}\right)\right) \\
& \text {if } u \in B V\left((-1 / 4,1 / 4) ; Z_{1}^{k} \cup Z_{2}^{k}\right) \text { and } W^{k}(x, u)=0 \text { a.e., } \\
&+\infty \quad \text { otherwise, }
\end{aligned}\right.
$$

where $C_{W}:=2 \int_{-1}^{1} \sqrt{W(s)} \mathrm{d} s$ and $u\left(0^{+}\right), u\left(0^{-}\right)$are the values taken a.e. by $u$ on $(0, r)$ and $(-r, 0)$, respectively, for $r>0$ small enough.

(ii) (Compatibility with integral constraint) Let $s \in \mathbb{R}$ and let $G_{\eta}^{k, s}$ be defined on $L^{2}(-1 / 4,1 / 4)$ by

$$
G_{\eta}^{k, s}(u)= \begin{cases}G_{\eta}^{k}(u) & \text { if } u \in W^{1,2}(-1 / 4,1 / 4) \text { and } f_{-1 / 4}^{1 / 4} u \mathrm{~d} x=s, \\ +\infty & \text { otherwise. }\end{cases}
$$

Then the family of functionals $G_{\eta}^{k, s} \Gamma$-converges with respect to strong $L^{2}$-convergence to the functional defined on $L^{2}(-1 / 4,1 / 4)$ by

$$
G^{k, s}(u)= \begin{cases}G^{k}(u) & \text { if } u \in L^{2}(-1 / 4,1 / 4) \text { and } f_{-1 / 4}^{1 / 4} u \mathrm{~d} x=s, \\ +\infty & \text { otherwise. }\end{cases}
$$

Proof. The proofs of (i) and (ii) exactly follow the lines of those of Theorems 6.4 and 6.6 in [3], with the only difference that now the zero set of the potential $W^{k}$ varies with $x$, being equal to $Z_{1}^{k}$ in $(0,1 / 4)$ and to $Z_{2}^{k}$ in $(-1 / 4,0)$, thus forcing sequences with equi-bounded energy to make an additional transition in an $\eta$-neighborhood of $x=0$.

COROLLARY 4.4 (convergence of minimum problems) For any fixed $\eta>0$ and for every $s \in \mathbb{R}$, let $\varphi_{\eta}^{k}$ be the function defined as

$$
\varphi_{\eta}^{k}(s):=\min \left\{\int_{-1 / 4}^{1 / 4}\left(\frac{1}{\eta} W^{k}(x, u)+\eta\left(u^{\prime}\right)^{2}\right) \mathrm{d} x: u \in W^{1,2}(-1 / 4,1 / 4), f_{-1 / 4}^{1 / 4} u \mathrm{~d} x=s\right\} .
$$

Then for every $s \in \mathbb{R}$,

$$
\lim _{\eta \rightarrow 0} \varphi_{\eta}^{k}(s)=\varphi^{k}(s)
$$

where

$$
\varphi^{k}(s)= \begin{cases}C_{1}^{k} & \text { if } s=-1 \text { or } 1, \\ C_{2}^{k} & \text { if } s=0, \\ C_{3}^{k} & \text { if } 0<|s|<1, k \leqslant 1 / 2, \\ C_{2}^{k}+C_{W} & \text { if } 0<|s|<1, k>1 / 2, \\ +\infty & \text { if }|s|>1 .\end{cases}
$$

Proof. We first observe that

$$
\begin{aligned}
\min G^{k, \pm 1} & =C_{1}^{k}, \quad \min G^{k, 0}=C_{2}^{k}, \\
\min G^{k, s} & =\left\{\begin{array}{ll}
C_{1}^{k}+C_{W}=C_{3}^{k} & \text { if } k \leqslant 1 / 2 \\
C_{2}^{k}+C_{W} & \text { if } k>1 / 2
\end{array} \quad \text { for } 0<|s|<1,\right.
\end{aligned}
$$


while the set of functions $u:(-1 / 4,1 / 4) \rightarrow \mathbb{R}$ such that

$$
u \in B V\left((0,1 / 4) ; Z_{1}^{k}\right), \quad u \in B V\left((-1 / 4,0) ; Z_{2}^{k}\right) \quad \text { and } \quad f_{-1 / 4}^{1 / 4} u=s \quad \text { with }|s|>1
$$

is empty. Then, since $G_{\eta}^{k, s} \stackrel{\Gamma}{\longrightarrow} G^{k, s}$ and $\left(G_{\eta}^{k, s}\right)$ is equi-coercive with respect to strong $L^{2}$ convergence, the desired result follows immediately from the general property of convergence of minimum values.

Proposition 4.5 Let $\varphi_{\eta}^{k}$ be the function defined as in 4.11 . Then

(i) $\varphi_{\eta}^{k}(s) \leqslant c$ for some $c>0$ independent of $\eta$ and for every $s$ such that $|s| \leqslant 1$;

(ii) if $|s| \leqslant 1$ and $v_{\eta}^{s}$ is a minimizing function for $\varphi_{\eta}^{k}(s)$ (i.e., a test function for which $\varphi_{\eta}^{k}(s)=$ $\left.\int_{-1 / 4}^{1 / 4}\left(\frac{1}{\eta} W^{k}\left(x, v_{\eta}^{s}\right)+\eta\left(v_{\eta}^{s}\right)^{2}\right) \mathrm{d} x\right)$, then there exists a constant $M>0$, independent of $\eta$, such that $\left\|v_{\eta}^{s}\right\|_{\infty} \leqslant M$.

Proof. (i) For every $s$ with $|s| \leqslant 1$, we exhibit a function $v_{\eta}^{s}$ such that $f_{-1 / 4}^{1 / 4} v_{\eta}^{s} \mathrm{~d} x=s$ and for which

$$
\int_{-1 / 4}^{1 / 4}\left(\frac{1}{\eta} W^{k}\left(x, v_{\eta}^{s}\right)+\eta\left(v_{\eta}^{s^{\prime}}\right)^{2}\right) \mathrm{d} x \leqslant c
$$

for some $c>0$.

For later references, we treat in detail the cases $s=0$ and $s= \pm 1$, while in the case $0<|s|<1$ we only give the idea of the construction of $v_{\eta}^{s}$.

We start with $s=0$; then as $v_{\eta}^{0}$ we take the function defined by

$$
v_{\eta}^{0}(x):= \begin{cases}v_{\eta}^{0,-}(x) & \text { if }-1 / 4 \leqslant x \leqslant 0 \\ v_{\eta}^{0,+}(x) & \text { if } 0<x \leqslant 1 / 4\end{cases}
$$

where $v_{\eta}^{0,-}, v_{\eta}^{0,+}$ respectively solve

$$
\min _{\substack{v \in W^{1,2}(-1 / 4,0) \\ v(0)=0}} \int_{-1 / 4}^{0}\left(\frac{1}{\eta}(v-1+k)^{2}+\eta\left(v^{\prime}\right)^{2}\right) \mathrm{d} x, \min _{\substack{v \in W^{1,2}(0,1 / 4) \\ v(0)=0}} \int_{0}^{1 / 4}\left(\frac{1}{\eta}(v+1-k)^{2}+\eta\left(v^{\prime}\right)^{2}\right) \mathrm{d} x ;
$$

or equivalently, the associated Cauchy problems

$$
\left\{\begin{array} { l } 
{ \eta ^ { 2 } v ^ { \prime \prime } - v + 1 - k = 0 \quad \text { in } ( - 1 / 4 , 0 ) , } \\
{ v ( 0 ) = 0 , \quad v ^ { \prime } ( - 1 / 4 ) = 0 , }
\end{array} \quad \left\{\begin{array}{l}
\eta^{2} v^{\prime \prime}-v-1+k=0 \quad \text { in }(0,1 / 4), \\
v(0)=0, \quad v^{\prime}(1 / 4)=0 .
\end{array}\right.\right.
$$

Hence, by directly solving the above equations we get

$$
v_{\eta}^{0}(x)= \begin{cases}1-k+(k-1) \cosh \left(\frac{x}{\eta}\right)+(k-1) \sinh \left(\frac{x}{\eta}\right) \tanh \left(\frac{1}{4 \eta}\right) & \text { if }-1 / 4 \leqslant x \leqslant 0, \\ -1+k-(k-1) \cosh \left(\frac{x}{\eta}\right)+(k-1) \sinh \left(\frac{x}{\eta}\right) \tanh \left(\frac{1}{4 \eta}\right) & \text { if } 0 \leqslant x \leqslant 1 / 4,\end{cases}
$$



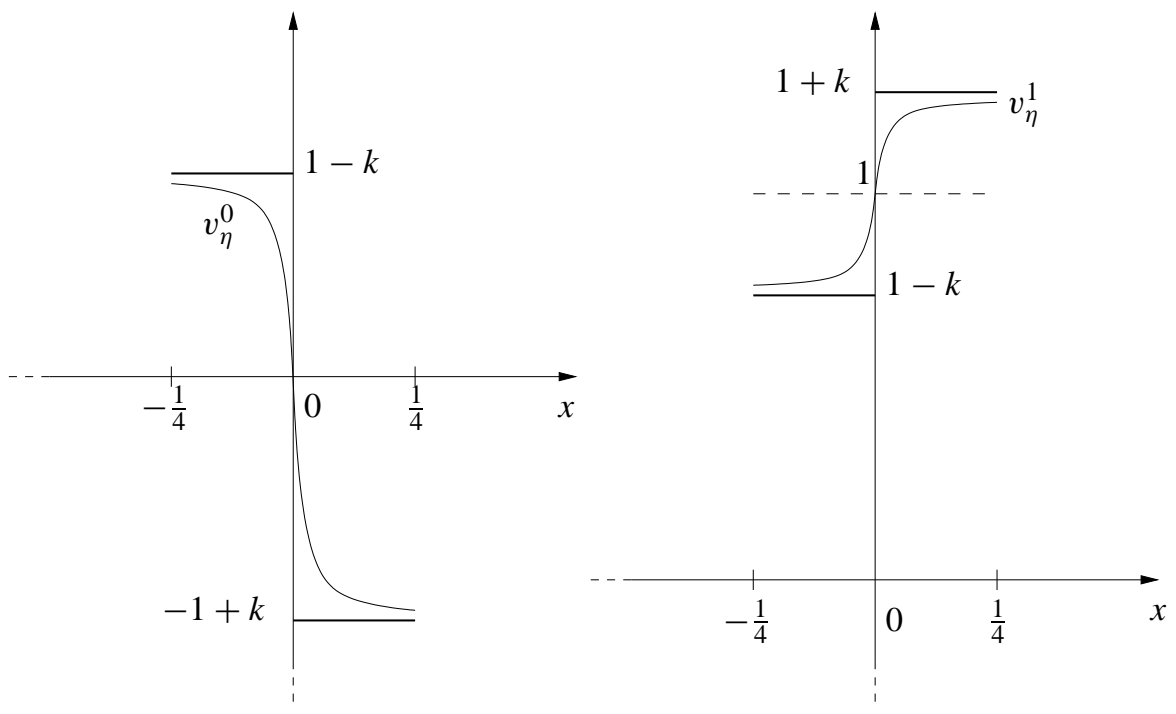

FIG. 9. The functions $v_{\eta}^{0}$ and $v_{\eta}^{1}$.

and thus immediately

$$
\int_{-1 / 4}^{1 / 4} v_{\eta}^{0} \mathrm{~d} x=0
$$

Moreover, a straightforward calculation gives

$$
\int_{-1 / 4}^{1 / 4}\left(\frac{1}{\eta} W^{k}\left(x, v_{\eta}^{0}\right)+\eta\left(\left(v_{\eta}^{0}\right)^{\prime}\right)^{2}\right) \mathrm{d} x=C_{2}^{k} \tanh \left(\frac{1}{4 \eta}\right),
$$

and finally

$$
\varphi_{\eta}^{k}(0) \leqslant C_{2}^{k} \tanh \left(\frac{1}{4 \eta}\right)<C_{2}^{k} \quad \text { for all } \eta>0 .
$$

If $s=1$, we proceed as above, now taking as a minimizing function for $\varphi_{\eta}^{k}(1)$,

$$
v_{\eta}^{1}(x):= \begin{cases}v_{\eta}^{1,-}(x) & \text { if }-1 / 4 \leqslant x \leqslant 0 \\ v_{\eta}^{1,+}(x) & \text { if } 0<x \leqslant 1 / 4\end{cases}
$$

where $v_{\eta}^{1,-}, v_{\eta}^{1,+}$ are respectively solutions to $\min _{\substack{v \in W^{1,2}(-1 / 4,0) \\ v(0)=1}} \int_{-1 / 4}^{0}\left(\frac{1}{\eta}(v-1+k)^{2}+\eta\left(v^{\prime}\right)^{2}\right) \mathrm{d} x, \min _{\substack{v \in W^{1,2}(0,1 / 4) \\ v(0)=1}} \int_{0}^{1 / 4}\left(\frac{1}{\eta}(v-1-k)^{2}+\eta\left(v^{\prime}\right)^{2}\right) \mathrm{d} x$, or to

$$
\left\{\begin{array} { l } 
{ \eta ^ { 2 } v ^ { \prime \prime } - v + 1 - k = 0 \quad \text { in } ( - 1 / 4 , 0 ) , } \\
{ v ( 0 ) = 1 , \quad v ^ { \prime } ( - 1 / 4 ) = 0 , }
\end{array} \quad \left\{\begin{array}{l}
\eta^{2} v^{\prime \prime}-v+1+k=0 \quad \text { in }(0,1 / 4), \\
v(0)=1, \quad v^{\prime}(1 / 4)=0
\end{array}\right.\right.
$$


Hence, we find

$$
v_{\eta}^{1}(x)= \begin{cases}1-k+k \cosh \left(\frac{x}{\eta}\right)+k \sinh \left(\frac{x}{\eta}\right) \tanh \left(\frac{1}{4 \eta}\right) & \text { if }-1 / 4 \leqslant x \leqslant 0, \\ 1+k-k \cosh \left(\frac{x}{\eta}\right)+k \sinh \left(\frac{x}{\eta}\right) \tanh \left(\frac{1}{4 \eta}\right) & \text { if } 0 \leqslant x \leqslant 1 / 4,\end{cases}
$$

and we have

$$
\int_{-1 / 4}^{1 / 4} v_{\eta}^{1} \mathrm{~d} x=1
$$

Then a direct computation gives

$$
\varphi_{\eta}^{k}(1) \leqslant \int_{-1 / 4}^{1 / 4}\left(\frac{1}{\eta} W^{k}\left(x, v_{\eta}^{1}\right)+\eta\left(\left(v_{\eta}^{1}\right)^{\prime}\right)^{2}\right) \mathrm{d} x=C_{1}^{k} \tanh \left(\frac{1}{4 \eta}\right)<C_{1}^{k} \quad \text { for all } \eta>0 .
$$

Notice that if $s=-1$, we simply take $v_{\eta}^{-1}:=v_{\eta}^{1}-2$.

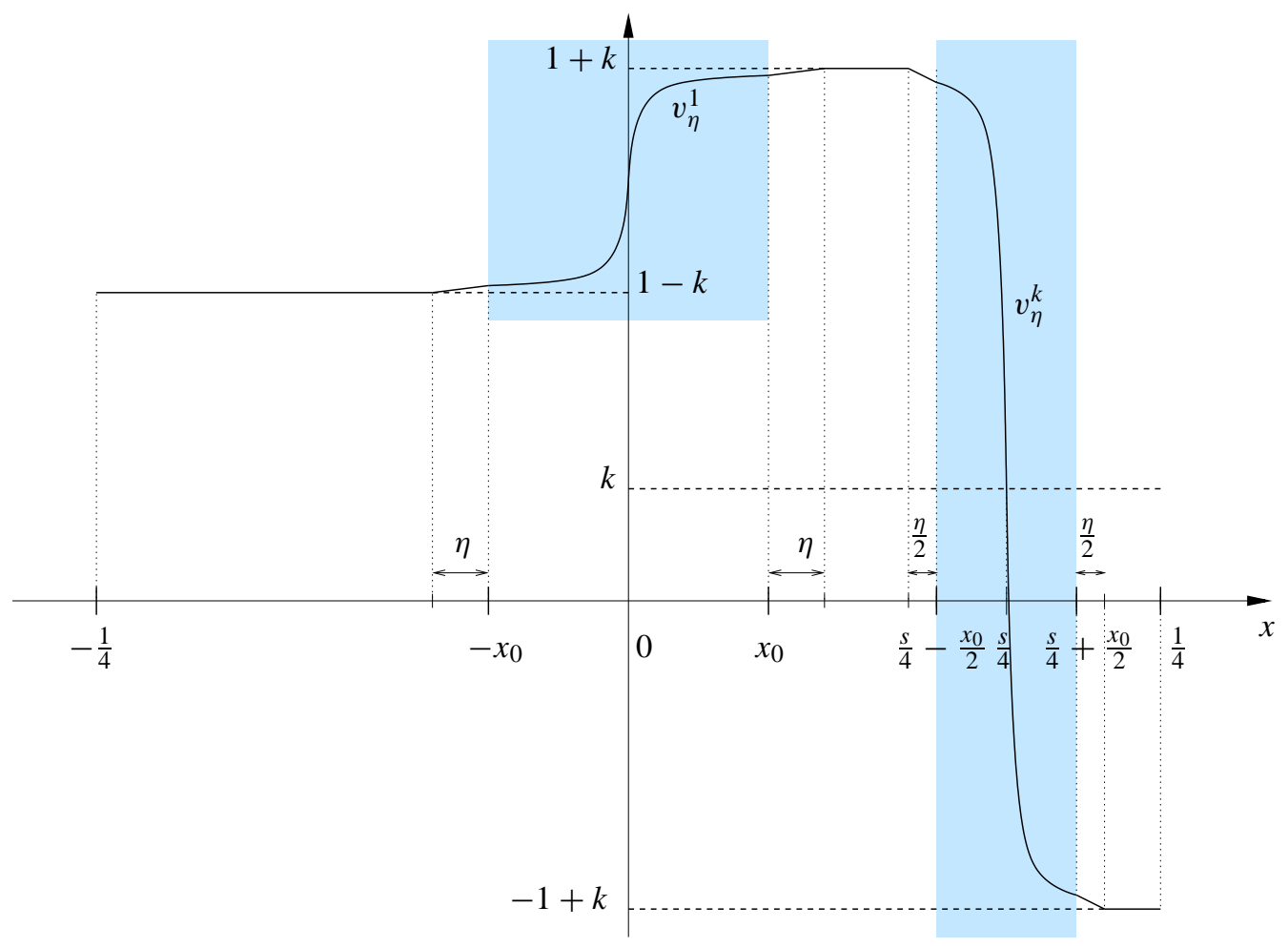

FIG. 10. The function $v_{\eta}^{s}$.

We now turn to the case $0<|s|<1$ and we sketch the proof for $s>0$, the one for $s<0$ being analogous. In this case a test function $v_{\eta}^{s}$ can be obtained as in Fig. 10 by suitably modifying $v_{\eta}^{1}$ 
and combining it, for instance, with an optimal transition $v_{\eta}^{k}$ between the two zeroes of the potential $W_{1}^{k}, 1+k$ and $-1+k$. More precisely, $v_{\eta}^{k}$ is defined by

$$
v_{\eta}^{k}(x):=v\left(\frac{x-s / 4}{\eta}\right)+k, \quad \frac{s}{4}-\frac{x_{0}}{2} \leqslant x \leqslant \frac{s}{4}+\frac{x_{0}}{2},
$$

where $v$ is the solution to the optimal profile problem

$$
\inf \left\{\int_{-\infty}^{+\infty}\left(W(u)+\left(u^{\prime}\right)^{2}\right) \mathrm{d} x: u(-\infty)=1, u(+\infty)=-1\right\}
$$

Then, as it can be easily checked that the energy contribution of the linear modification to $v_{\eta}^{1}$ and $v_{\eta}^{k}$ is (exponentially) small as $\eta \rightarrow 0$, we get

$$
\varphi_{\eta}^{k}(s) \leqslant C_{1}^{k}+C_{W}+o(1) \quad \text { as } \eta \rightarrow 0,
$$

and thus $\varphi_{\eta}^{k}$ is bounded.

We remark that the last construction is not "optimal" since the bound on $\varphi_{\eta}^{k}$ can be improved for $0<|s|<1$ to

$$
\varphi_{\eta}^{k}(s) \leqslant \min \left\{C_{3}^{k}, C_{2}^{k}+C_{W}\right\} \quad \text { for all } \eta>0 .
$$

(ii) Let $|s| \leqslant 1$ and let $v_{\eta}^{s} \in W^{1,2}(-1 / 4,1 / 4)$ be a minimizing function for $\varphi_{\eta}^{k}(s)$. We argue by contradiction, supposing the existence of a point $x^{\prime} \in(-1 / 4,1 / 4)$ such that

$$
v_{\eta}^{s}\left(x^{\prime}\right)>M \geqslant 3(1+k) .
$$

To fix ideas, and without loss of generality, we may additionally assume that $x^{\prime} \in(0,1 / 4)$.

Now, appealing to (i) we have for instance

$$
\varphi_{\eta}^{k}(s)=\int_{-1 / 4}^{1 / 4}\left(\frac{1}{\eta} W^{k}\left(x, v_{\eta}^{s}\right)+\eta\left(v_{\eta}^{s \prime}\right)^{2}\right) \mathrm{d} x \leqslant C_{3}^{k}
$$

and from this we deduce that the restriction of $v_{\eta}^{s}$ to $(0,1 / 4)$ converges in measure to $Z_{1}^{k}$ as $\eta \rightarrow 0$. In fact, for any fixed $\sigma>0$,

$$
\left|\left\{x \in(0,1 / 4): \operatorname{dist}\left(v_{\eta}^{s}(x), Z_{1}^{k}\right)>\sigma\right\}\right| \min \{W(\tau):|| \tau|-1|>\eta\} \leqslant C_{3}^{k} \eta \rightarrow 0 \quad \text { as } \eta \rightarrow 0 .
$$

Thus, for sufficiently small $\eta>0$ there exists $x^{\prime \prime} \in(0,1 / 4)$ such that

$$
\min \left\{\left|v_{\eta}^{s}\left(x^{\prime \prime}\right)-(1+k)\right|,\left|v_{\eta}^{s}\left(x^{\prime \prime}\right)-(-1+k)\right|\right\} \leqslant \sigma .
$$

We may suppose that $\left|v_{\eta}^{s}\left(x^{\prime \prime}\right)-(1+k)\right| \leqslant \sigma$, and get

$$
v_{\eta}^{s}\left(x^{\prime \prime}\right) \leqslant 2(1+k)
$$

having also chosen $\sigma=1+k$. 
Finally, using the so-called Modica-Mortola trick together with (4.14) and 4.15), we get

$$
\begin{aligned}
\varphi_{\eta}^{k}(s) & \geqslant \int_{0}^{1 / 4}\left(\frac{1}{\eta} W_{1}^{k}\left(v_{\eta}^{s}\right)+\eta\left(\left(v_{\eta}^{s}\right)^{\prime}\right)^{2}\right) \mathrm{d} x \geqslant 2 \int_{v_{\eta}^{s}\left(x^{\prime \prime}\right)}^{v_{\eta}^{s}\left(x^{\prime}\right)} \sqrt{W_{1}^{k}(s)} \mathrm{d} s \\
& >\int_{2(1+k)}^{M} 2(s-1-k) \mathrm{d} s=M^{2}-2 M(1+k) \geqslant 3(1+k)^{2}>C_{3}^{k}
\end{aligned}
$$

and thus a contradiction.

Notice that if $v_{\eta}^{s}$ converges in measure to the constant $-1+k$, then since $-1+k<1+k$, the same argument again applies to yield the conclusion.

In all that follows, the letter $C$ will stand for a generic strictly positive constant which may vary from line to line and expression to expression within the same formula.

Proof of Theorem 4.2 Step 1: $\Gamma$-liminf inequality. We have to prove that if $u_{\varepsilon} \rightarrow u$ in $L^{2}(0,1)$, then $F^{k(1)}(u) \leqslant \liminf _{\varepsilon \rightarrow 0} F_{\varepsilon}^{k(1)}\left(u_{\varepsilon}\right)$. Notice that if moreover $\sup _{\varepsilon} F_{\varepsilon}^{k(1)}\left(u_{\varepsilon}\right)<+\infty$ then, by the definition of $F_{\varepsilon}^{k(1)},|u| \leqslant 1$ a.e.

By virtue of the nonnegative character of $W^{k}$, we have

$$
\begin{aligned}
F_{\varepsilon}^{k(1)}\left(u_{\varepsilon}\right) & =\int_{0}^{1}\left(\frac{\delta}{\varepsilon} W^{k}\left(\frac{x}{\delta}, u_{\varepsilon}\right)+\varepsilon \delta\left(u_{\varepsilon}^{\prime}\right)^{2}\right) \mathrm{d} x \\
& \geqslant \sum_{i=1}^{[2 / \delta-1 / 2]} \int_{(2 i-1) \delta / 4}^{(2 i+1) \delta / 4}\left(\frac{\delta}{\varepsilon} W^{k}\left(\frac{x}{\delta}, u_{\varepsilon}\right)+\varepsilon \delta\left(u_{\varepsilon}^{\prime}\right)^{2}\right) \mathrm{d} x ;
\end{aligned}
$$

then, by the change of variable $x=\delta t+(\delta / 2) i$, denoting by $[r]$ the integer part of $r \in \mathbb{R}$ and setting

$$
v_{\varepsilon}^{i}(t):=u_{\varepsilon}(\delta(t+i / 2)), \quad i=1, \ldots,[2 / \delta-1 / 2],
$$

we get

$$
\begin{aligned}
F_{\varepsilon}^{k(1)}\left(u_{\varepsilon}\right) \geqslant & \sum_{i=1}^{[2 / \delta-1 / 2]} \delta \int_{-1 / 4}^{1 / 4}\left(\frac{\delta}{\varepsilon} W^{k}\left(t+\frac{i}{2}, v_{\varepsilon}^{i}\right)+\frac{\varepsilon}{\delta}\left(\left(v_{\varepsilon}^{i}\right)^{\prime}\right)^{2}\right) \mathrm{d} t \\
= & \sum_{i \text { even }} \delta \int_{-1 / 4}^{1 / 4}\left(\frac{\delta}{\varepsilon} W^{k}\left(t, v_{\varepsilon}^{i}\right)+\frac{\varepsilon}{\delta}\left(\left(v_{\varepsilon}^{i}\right)^{\prime}\right)^{2}\right) \mathrm{d} t \\
& +\sum_{i \text { odd }} \delta \int_{1 / 4}^{3 / 4}\left(\frac{\delta}{\varepsilon} W^{k}\left(t, w_{\varepsilon}^{i}\right)+\frac{\varepsilon}{\delta}\left(\left(w_{\varepsilon}^{i}\right)^{\prime}\right)^{2}\right) \mathrm{d} t
\end{aligned}
$$

where $w_{\varepsilon}^{i}(t):=v_{\varepsilon}^{i}(t-1 / 2)$. We observe that

$$
\begin{aligned}
\min \left\{\int_{-1 / 4}^{1 / 4}\left(\frac{\delta}{\varepsilon} W^{k}(t, v)+\frac{\varepsilon}{\delta}\left(v^{\prime}\right)^{2}\right) \mathrm{d} t:\right. & \left.f_{-1 / 4}^{1 / 4} v \mathrm{~d} t=s\right\} \\
& =\min \left\{\int_{1 / 4}^{3 / 4}\left(\frac{\delta}{\varepsilon} W^{k}(t, v)+\frac{\varepsilon}{\delta}\left(v^{\prime}\right)^{2}\right) \mathrm{d} t: f_{1 / 4}^{3 / 4} v \mathrm{~d} t=s\right\},
\end{aligned}
$$


and as a consequence we find

$$
F_{\varepsilon}^{k(1)}\left(u_{\varepsilon}\right) \geqslant \sum_{i=1}^{[2 / \delta-1 / 2]} \delta \min \left\{\int_{-1 / 4}^{1 / 4}\left(\frac{\delta}{\varepsilon} W^{k}(t, v)+\frac{\varepsilon}{\delta}\left(v^{\prime}\right)^{2}\right) \mathrm{d} t: \int_{-1 / 4}^{1 / 4} v \mathrm{~d} t=f_{(2 i-1) \delta / 4}^{(2 i+1) \delta / 4} u_{\varepsilon} \mathrm{d} t\right\} .
$$

Hence, by using the notation introduced in Corollary 4.4, 4.16, becomes

$$
F_{\varepsilon}^{k(1)}\left(u_{\varepsilon}\right) \geqslant 2 \sum_{i=1}^{[2 / \delta-1 / 2]} \frac{\delta}{2} \varphi_{\varepsilon / \delta}^{k}\left(f_{(2 i-1) \delta / 4}^{(2 i+1) \delta / 4} u_{\varepsilon} \mathrm{d} t\right)
$$

and if we define $\tilde{u}_{\varepsilon}:(0,1) \rightarrow \mathbb{R}$ as

$$
\tilde{u}_{\varepsilon}(x):=\sum_{i=1}^{[2 / \delta-1 / 2]}\left(f_{(2 i-1) \delta / 4}^{(2 i+1) \delta / 4} u_{\varepsilon} \mathrm{d} t\right) \chi_{((2 i-1) \delta / 4,(2 i+1) \delta / 4)}(x),
$$

we finally have, using the uniform boundedness of $\varphi_{\varepsilon / \delta}^{k}$,

$$
\liminf _{\varepsilon \rightarrow 0} F_{\varepsilon}^{k(1)}\left(u_{\varepsilon}\right) \geqslant 2 \liminf _{\varepsilon \rightarrow 0} \int_{0}^{1} \varphi_{\varepsilon / \delta}^{k}\left(\tilde{u}_{\varepsilon}\right) \mathrm{d} x .
$$

Note, moreover, that $\tilde{u}_{\varepsilon} \rightarrow u$ in $L^{2}(0,1)$.

Now our goal is to estimate $\varphi_{\varepsilon / \delta}^{k}$ from below. To this end we first consider the case $|s|>1$. On one hand (see also (3.6)), for every $s \in \mathbb{R}$ we have

$$
\begin{aligned}
\varphi_{\varepsilon / \delta}^{k}(s) & \geqslant \inf \left\{\frac{\delta}{\varepsilon} \int_{-1 / 4}^{1 / 4} W^{k}(t, v) \mathrm{d} t: \int_{-1 / 4}^{1 / 4} v \mathrm{~d} t=s\right\} \\
& =\frac{\delta}{\varepsilon} \min \left\{\frac{1}{4} W^{* *}\left(s_{1}+k\right)+\frac{1}{4} W^{* *}\left(s_{2}-k\right): s_{1}+s_{2}=2 s\right\}=\frac{\delta}{\varepsilon} \frac{W^{* *}(s)}{2},
\end{aligned}
$$

so in particular

$$
\varphi_{\varepsilon / \delta}^{k}(s) \geqslant \frac{\delta}{\varepsilon} \frac{(|s|-1)^{2}}{2} \quad \text { for all } s \text { such that }|s|>1 .
$$

On the other hand, for any fixed $\eta>0$ there exist $\sigma, \varepsilon_{0}>0$ such that

$$
\varphi_{\varepsilon / \delta}^{k}(s) \geqslant C_{1}^{k}-\eta^{2} \quad \text { for all } s \in(1,1+\sigma) \text { and } \varepsilon<\varepsilon_{0},
$$

which can be proved by the following argument. If 4.18 does not hold we can find two sequences $s_{n} \rightarrow 1$ and $\varepsilon_{n} \rightarrow 0$ for which

$$
\varphi_{\varepsilon_{n} / \delta\left(\varepsilon_{n}\right)}^{k}\left(s_{n}\right)<C_{1}^{k}-\eta_{0}^{2}
$$

for every $n \in \mathbb{N}$ and for some $\eta_{0}>0$. Appealing to Corollary 4.4 we can also deduce

$$
C_{1}^{k}=\varphi^{k}(1) \leqslant \liminf _{n \rightarrow+\infty} \varphi_{\varepsilon_{n} / \delta\left(\varepsilon_{n}\right)}^{k}\left(s_{n}\right),
$$

and combining it with 4.19 we find a contradiction. Note that, by symmetry, 4.18) also holds true for every $s \in(-1-\sigma,-1)$. Hence, combining 4.17) and 4.18) we deduce that for every $\eta>0$ and for any sufficiently small $\varepsilon>0$,

$$
\varphi_{\varepsilon / \delta}^{k}(s) \geqslant\left(C_{1}^{k}-\eta^{2}\right) \vee\left(\frac{\delta}{\varepsilon} \frac{(|s|-1)^{2}}{2}\right) \quad \text { for all } s \text { such that }|s|>1 .
$$


Now it remains to estimate $\varphi_{\varepsilon / \delta}^{k}$ for $|s| \leqslant 1$. To this end, for any fixed $\eta>0$, consider the set

$$
A_{\eta}^{\varepsilon}:=\left\{t \in(-1 / 4,1 / 4): \operatorname{dist}\left(v_{\varepsilon}^{s}(t), Z^{k}(t)\right)>\eta\right\},
$$

where $v_{\varepsilon}^{s}$ is a minimizing function for $\varphi_{\varepsilon / \delta}^{k}(s)$ and

$$
Z^{k}(t):= \begin{cases}Z_{2}^{k} & \text { if } t \in(-1 / 4,0) \\ Z_{1}^{k} & \text { if } t \in(0,1 / 4)\end{cases}
$$

Then, arguing as in the proof of Proposition 4.5 (ii), we deduce that the measure of $A_{\eta}^{\varepsilon}$ tends to zero as $\varepsilon \rightarrow 0$. In fact, we have

$$
\left|A_{\eta}^{\varepsilon}\right| \min \{W(\tau):|| \tau|-1|>\eta\} \leqslant \frac{\varepsilon}{\delta} C_{3}^{k} \rightarrow 0 \quad \text { as } \varepsilon \rightarrow 0 .
$$

As a consequence, for any sufficiently small $\varepsilon>0$ we can find $t^{-} \in(-1 / 4,0)$ and $t^{+} \in(0,1 / 4)$ such that $\operatorname{dist}\left(v_{\varepsilon}^{s}\left(t^{ \pm}\right), Z^{k}\left(t^{ \pm}\right)\right) \leqslant \eta$.

If one of the following inequalities holds true:

$$
\left|v_{\varepsilon}^{s}\left(t^{-}\right)-(-1-k)\right| \leqslant \eta, \quad\left|v_{\varepsilon}^{s}\left(t^{+}\right)-(1+k)\right| \leqslant \eta,
$$

say the first, we deduce

$$
\varphi_{\varepsilon / \delta}^{k}(s)=\int_{-1 / 4}^{1 / 4}\left(\frac{\delta}{\varepsilon} W^{k}\left(t, v_{\varepsilon}^{s}\right)+\frac{\varepsilon}{\delta}\left(v_{\varepsilon}^{s^{\prime}}\right)^{2}\right) \mathrm{d} t \geqslant C_{W^{k}}(-1-k+\eta,-1+k-\eta),
$$

with $C_{W^{k}}(\cdot, \cdot)$ as in 4.6 , and eventually

$$
\varphi_{\varepsilon / \delta}^{k}(s) \geqslant C_{1}^{k}-C \eta^{2} .
$$

We will now prove that whenever $4 \eta<|s| \leqslant 1$, at least one of the inequalities in 4.21 is fulfilled. Arguing by contradiction we can find a number $\eta_{0}>0$ and a sequence $\varepsilon_{n} \rightarrow 0$ such that for every $n \in \mathbb{N}$,

$$
\left|v_{\varepsilon_{n}}^{s}(t)-(-1-k)\right|>\eta_{0} \quad \text { for } t \in(-1 / 4,0), \quad\left|v_{\varepsilon_{n}}^{s}(t)-(1+k)\right|>\eta_{0} \quad \text { for } t \in(0,1 / 4) .
$$

If we set

$$
Z_{0}^{k}(t):= \begin{cases}1-k & \text { if } t \in(-1 / 4,0), \\ -1+k & \text { if } t \in(0,1 / 4),\end{cases}
$$

then in view of $4.23, A_{\eta_{0}}^{\varepsilon_{n}}$ can be rewritten as

$$
A_{\eta_{0}}^{\varepsilon_{n}}=\left\{t \in(-1 / 4,1 / 4): \operatorname{dist}\left(v_{\varepsilon_{n}}^{s}(t), Z_{0}^{k}(t)\right)>\eta_{0}\right\},
$$

and again the complement of $A_{\eta_{0}}^{\varepsilon_{n}}$ decomposes as

$$
\left(A_{\eta_{0}}^{\varepsilon_{n}}\right)^{c}=B_{\eta_{0}}^{\varepsilon_{n},-} \cup B_{\eta_{0}}^{\varepsilon_{n},+}
$$


where

$$
\begin{aligned}
& B_{\eta_{0}}^{\varepsilon_{n},-}:=\left\{t \in(-1 / 4,0):\left|v_{\varepsilon_{n}}^{s}(t)-(1-k)\right| \leqslant \eta_{0}\right\}, \\
& B_{\eta_{0}}^{\varepsilon_{n},+}:=\left\{t \in(0,1 / 4):\left|v_{\varepsilon_{n}}^{s}(t)-(-1+k)\right| \leqslant \eta_{0}\right\}
\end{aligned}
$$

and

$$
\left|B_{\eta_{0}}^{\varepsilon_{n},-}\right|-\left|B_{\eta_{0}}^{\varepsilon_{n},+}\right| \rightarrow 0 \quad \text { as } n \rightarrow+\infty .
$$

Without loss of generality, we can suppose $s>0$; then

$$
2 \eta_{0}<\int_{-1 / 4}^{1 / 4} v_{\varepsilon_{n}}^{s} \mathrm{~d} t=\int_{A_{\eta_{0}}^{\varepsilon_{n}}} v_{\varepsilon_{n}}^{s} \mathrm{~d} t+\int_{\left(A_{\eta_{0}}^{\varepsilon_{n}}\right)^{c}} v_{\varepsilon_{n}}^{s} \mathrm{~d} t
$$

Now by (4.24), 4.25) and Proposition 4.5 (ii), we deduce

$$
\begin{aligned}
2 \eta_{0} & <\int_{A_{\eta_{0}}^{\varepsilon_{n}}} v_{\varepsilon_{n}}^{s} \mathrm{~d} t+\int_{B_{\eta_{0}}^{\varepsilon_{n},-}} v_{\varepsilon_{n}}^{s} \mathrm{~d} t+\int_{B_{\eta_{0}}^{\varepsilon_{n},+}} v_{\varepsilon_{n}}^{s} \mathrm{~d} t \\
& \leqslant M\left|A_{\eta_{0}}^{\varepsilon_{n}}\right|+\left(\eta_{0}+(1-k)\right)\left|B_{\eta_{0}}^{\varepsilon_{n},-}\right|+\left(\eta_{0}+(-1+k)\right)\left|B_{\eta_{0}}^{\varepsilon_{n},+}\right| \\
& \leqslant M\left|A_{\eta_{0}}^{\varepsilon_{n}}\right|+\eta_{0} / 2+(1-k)\left(\left|B_{\eta_{0}}^{\varepsilon_{n},-}\right|-\left|B_{\eta_{0}}^{\varepsilon_{n},+}\right|\right) ;
\end{aligned}
$$

moreover by 4.26 , for any sufficiently large $n$, we have

$$
\left|A_{\eta_{0}}^{\varepsilon_{n}}\right|>\eta_{0} / M
$$

which yields a contradiction. Then for $|s| \leqslant 4 \eta$ it is easy to check that

$$
\varphi_{\varepsilon / \delta(s)}^{k} \geqslant C_{2}^{k}-C \eta^{2} .
$$

Finally, combining (4.20), 4.22) and 4.27] we get

$$
\varphi_{\varepsilon / \delta}^{k}(s) \geqslant \psi_{\eta, \delta / \varepsilon}^{k}(s):= \begin{cases}C_{2}^{k}-C \eta^{2} & \text { if }|s| \leqslant \eta, \\ C_{1}^{k}-C \eta^{2} & \text { if } \eta<|s| \leqslant 1, \\ \left(C_{1}^{k}-C \eta^{2}\right) \vee\left(\frac{\delta}{\varepsilon} \frac{(|s|-1)^{2}}{2}\right) & \text { if }|s|>1,\end{cases}
$$

for every $s \in \mathbb{R}$ and for every $0<\eta<1$; hence

$$
\liminf _{\varepsilon \rightarrow 0} F_{\varepsilon}^{k(1)}\left(u_{\varepsilon}\right) \geqslant \liminf _{\varepsilon \rightarrow 0} 2 \int_{0}^{1} \psi_{\eta, \delta / \varepsilon}^{k}\left(\tilde{u}_{\varepsilon}\right) \mathrm{d} x .
$$

To conclude the proof, we note that, for any fixed $s \in \mathbb{R}$, the sequence $\left(\psi_{\eta, \delta / \varepsilon}^{k}(s)\right)$ increases with $\delta / \varepsilon$, so in particular for every $m>0$, there exists $\varepsilon_{0}>0$ such that

$$
\psi_{\eta, \delta / \varepsilon}^{k}(s) \geqslant \psi_{\eta, m}^{k}(s) \quad \text { for all } s \in \mathbb{R} \text { and } \varepsilon \leqslant \varepsilon_{0} .
$$

Then

$$
\begin{aligned}
\liminf _{\varepsilon \rightarrow 0} \int_{0}^{1} \psi_{\eta, \delta / \varepsilon}^{k}\left(\tilde{u}_{\varepsilon}\right) \mathrm{d} x & \geqslant \liminf _{\varepsilon \rightarrow 0} \int_{0}^{1} \psi_{\eta, m}^{k}\left(\tilde{u}_{\varepsilon}\right) \mathrm{d} x \\
& \geqslant \liminf _{\varepsilon \rightarrow 0} \int_{0}^{1}\left(\psi_{\eta, m}^{k}\right)^{* *}\left(\tilde{u}_{\varepsilon}\right) \mathrm{d} x \geqslant \int_{0}^{1}\left(\psi_{\eta, m}^{k}\right)^{* *}(u) \mathrm{d} x,
\end{aligned}
$$


using the fact that $\tilde{u}_{\varepsilon} \rightarrow u$ in $L^{2}(0,1)$ and the $L^{2}$-weak lower semicontinuity of $u \mapsto$ $\int_{0}^{1}\left(\psi_{\eta, m}^{k}\right)^{* *}(u) \mathrm{d} x$ in the last inequality. Moreover, by the Monotone Convergence Theorem,

$$
\lim _{m \rightarrow+\infty} \int_{0}^{1}\left(\psi_{\eta, m}^{k}\right)^{* *}(u) \mathrm{d} x=\int_{0}^{1} \lim _{m \rightarrow+\infty}\left(\psi_{\eta, m}^{k}\right)^{* *}(u) \mathrm{d} x=\int_{0}^{1}\left(\psi_{\eta}^{k}\right)(u) \mathrm{d} x,
$$

where

$$
\psi_{\eta}^{k}(s):=C_{1}^{k}-C \eta^{2} \quad \text { if }|s| \leqslant 1 \quad \text { for } k \leqslant 1 / 2
$$

and

$$
\psi_{\eta}^{k}(s)=\left\{\begin{array}{ll}
C_{2}^{k}-C \eta^{2} & \text { if }|s| \leqslant \eta \\
\frac{C_{1}^{k}-C_{2}^{k}}{1-\eta}|s|+C_{2}^{k}-\frac{C_{1}^{k}-C_{2}^{k}}{1-\eta} \eta-C \eta^{2} & \text { if } \eta<|s| \leqslant 1
\end{array} \quad \text { for } k>1 / 2 .\right.
$$

Collecting these inequalities we find that

$$
\Gamma-\liminf _{\varepsilon \rightarrow 0} F_{\varepsilon}^{k(1)}(u) \geqslant 2 \int_{0}^{1} \psi_{\eta}^{k}(u) \mathrm{d} x,
$$

and by the arbitrariness of $\eta$,

$$
\Gamma-\liminf _{\varepsilon \rightarrow 0} F_{\varepsilon}^{k(1)}(u) \geqslant 2 \sup _{\eta>0} \int_{0}^{1} \psi_{\eta}^{k}(u) \mathrm{d} x .
$$

Hence, again applying the Monotone Convergence Theorem we obtain the desired result for both $k \leqslant 1 / 2$ and $k>1 / 2$.

Step 2: $\Gamma$-limsup inequality. To check the limsup inequality for the $\Gamma$-limit, it will suffice to deal with the case of a constant target function $u \equiv c(-1 \leqslant c \leqslant 1)$, since by repeating that construction we can easily deal with the case of $u$ piecewise constant and then the general case follows by density.

We start by approximating $c=1$. Fix $\eta>0$; by (4.2) and (4.5) there exist $T_{1}, T_{2}>0$ and $v_{1} \in W^{1,2}\left(-T_{1}, T_{2}\right)$ such that $v_{1}\left(-T_{1}\right)=1+k, v_{1}\left(T_{2}\right)=1-k$ and

$$
\int_{-T_{1}}^{0}\left(W_{1}^{k}\left(v_{1}\right)+\left(v_{1}^{\prime}\right)^{2}\right) \mathrm{d} x+\int_{0}^{T_{2}}\left(W_{2}^{k}\left(v_{1}\right)+\left(v_{1}^{\prime}\right)^{2}\right) \mathrm{d} x \leqslant C_{1}^{k}+\frac{\eta}{2} .
$$

Note that it is not restrictive to suppose $T_{1}=T_{2}=: T$. Then as a recovery sequence we can take, for instance,

$$
u_{\varepsilon}(x)= \begin{cases}1+k & \text { if } 0<x \leqslant \delta / 4, \\ v_{\varepsilon, 1}^{i}(x) & \text { if }(4 i-3) \delta / 4<x<(4 i+1) \delta / 4, \\ 1+k & \text { if }(4[1 / \delta-1 / 4]+1) \delta / 4 \leqslant x<1,\end{cases}
$$

for $i=1, \ldots,[1 / \delta-1 / 4]$, where

$$
v_{\varepsilon, 1}^{i}(x)= \begin{cases}1+k & \text { if }(4 i-3) \delta / 4<x<(2 i-1) \delta / 2-\varepsilon T, \\ v_{1}\left(\frac{x-(2 i-1) \delta / 2}{\varepsilon}\right) & \text { if }(2 i-1) \delta / 2-\varepsilon T \leqslant x \leqslant(2 i-1) \delta / 2+\varepsilon T, \\ 1-k & \text { if }(2 i-1) \delta / 2+\varepsilon T<x<i \delta-\varepsilon T, \\ v_{1}\left(\frac{i \delta-x}{\varepsilon}\right) & \text { if } i \delta-\varepsilon T \leqslant x \leqslant i \delta+\varepsilon T, \\ 1+k & \text { if } i \delta+\varepsilon T<x<(4 i+1) \delta / 4,\end{cases}
$$


for $i \in \mathbb{N}$. In fact, recalling that $\varepsilon \ll \delta$ it is easy to check that $u_{\varepsilon} \rightarrow 1$ in $L^{2}(0,1)$, while

$$
\begin{aligned}
\limsup _{\varepsilon \rightarrow 0} F_{\varepsilon}^{k(1)}\left(u_{\varepsilon}\right) & =\limsup _{\varepsilon \rightarrow 0} \sum_{i=1}^{[1 / \delta-1 / 4]} \int_{(4 i-3) \delta / 4}^{(4 i+1) \delta / 4}\left(\frac{\delta}{\varepsilon} W^{k}\left(\frac{x}{\delta}, v_{\varepsilon, 1}^{i}\right)+\varepsilon \delta\left(\left(v_{\varepsilon, 1}^{i}\right)^{\prime}\right)^{2}\right) \mathrm{d} x \\
& \leqslant \lim _{\varepsilon \rightarrow 0}\left[\frac{1}{\delta}-\frac{1}{4}\right] \delta\left(2 C_{1}^{k}+\eta\right)=2 C_{1}^{k}+\eta \quad \text { for all } \eta>0
\end{aligned}
$$

implies that

$$
\limsup _{\varepsilon \rightarrow 0} F_{\varepsilon}^{k(1)}\left(u_{\varepsilon}\right) \leqslant F^{k(1)}(1)
$$

If we replace $1 \pm k$ with $-1 \pm k$ and $v_{1}$ with its analogue $v_{-1}$, a similar construction yields $v_{\varepsilon,-1}^{i}$ and consequently the $\Gamma$-limsup for $c \equiv-1$.

If $-1<c<1$, it is necessary to make a distinction between the cases $k \leqslant 1 / 2$ and $k>1 / 2$. Let $k \leqslant 1 / 2$ and write $c$ as a convex combination of 1 and -1 ,

$$
c=\frac{1+c}{2} \cdot 1+\frac{1-c}{2} \cdot(-1)
$$

Now let $\left(n_{1}^{v}\right),\left(n_{2}^{v}\right)$ be two sequences of positive integers such that

$$
n_{1}^{v}, n_{2}^{v} \rightarrow+\infty \quad \text { and } \quad \frac{n_{1}^{v}}{n_{2}^{v}} \rightarrow \frac{1+c}{1-c} \quad \text { as } v \rightarrow 0 \text {. }
$$

With fixed $v>0$, for every sufficiently small $\varepsilon>0$ we have $\left(n_{1}^{v}+n_{2}^{v}+1\right) \delta \ll 1$. We consider the $\left(n_{1}^{v}+n_{2}^{v}+1\right) \delta$-periodic function $U_{\varepsilon}^{v}$, on $\mathbb{R}^{+}$, which on $\left(\delta / 4,\left(4\left(n_{1}^{v}+n_{2}^{v}\right)+5\right) \delta / 4\right)$ is defined as

$$
U_{\varepsilon}^{v}(x)= \begin{cases}v_{\varepsilon, 1}^{i}(x) & \text { if } x \in((4 i-3) \delta / 4,(4 i+1) \delta / 4) \text { for } i=1, \ldots, n_{1}^{v}, \\ w_{\varepsilon}(x) & \text { if } x \in\left(\left(4 n_{1}^{v}+1\right) \delta / 4,\left(4 n_{1}^{v}+5\right) \delta / 4\right), \\ v_{\varepsilon,-1}^{i}(x) & \text { if } x \in((4 i-3) \delta / 4,(4 i+1) \delta / 4) \text { for } i=n_{1}^{v}+2, \ldots, n_{1}^{v}+n_{2}^{v}, \\ \tilde{w}_{\varepsilon}(x) & \text { if } x \in\left(\left(4\left(n_{1}^{v}+n_{2}^{v}\right)+1\right) \delta / 4,\left(4\left(n_{1}^{v}+n_{2}^{v}\right)+5\right) \delta / 4\right),\end{cases}
$$

where $v_{\varepsilon, 1}^{i}$ is as in 4.28 and $v_{\varepsilon,-1}^{i}$ is analogous. Moreover, $w_{\varepsilon}$ is given by

$$
w_{\varepsilon}(x)= \begin{cases}v_{\varepsilon, 1}^{n_{1}^{v}+1}(x) & \text { if }\left(4 n_{1}^{v}+1\right) \delta / 4<x \leqslant\left(2 n_{1}^{v}+1\right) \delta / 2+\varepsilon T, \\ 1-k & \text { if }\left(2 n_{1}^{v}+1\right) \delta / 2+\varepsilon T<x<\left(n_{1}^{v}+1\right) \delta / 2-\varepsilon T^{\prime}, \\ v_{0}\left(\frac{x-\left(n_{1}^{v}+1\right) \delta}{\varepsilon}\right) & \text { if }\left(n_{1}^{v}+1\right) \delta-\varepsilon T^{\prime} \leqslant x \leqslant\left(n_{1}^{v}+1\right) \delta+\varepsilon T^{\prime}, \\ -1+k & \text { if }\left(n_{1}^{v}+1\right) \delta+\varepsilon T^{\prime}<x<\left(4 n_{1}^{v}+5\right) \delta / 4,\end{cases}
$$

with $T^{\prime}>0$ and $v_{0} \in W^{1,2}\left(-T^{\prime}, T^{\prime}\right)$ such that $v_{0}\left(-T^{\prime}\right)=1-k, v_{0}\left(T^{\prime}\right)=-1+k$ and

$$
\int_{-T^{\prime}}^{0}\left(W_{1}^{k}\left(v_{0}\right)+\left(v_{0}^{\prime}\right)^{2}\right) \mathrm{d} x+\int_{0}^{T^{\prime}}\left(W_{2}^{k}\left(v_{0}\right)+\left(v_{0}^{\prime}\right)^{2}\right) \mathrm{d} x \leqslant C_{2}^{k}+\frac{\eta}{2}
$$


while $\tilde{w}_{\varepsilon}$ is defined as

$$
\tilde{w}_{\varepsilon}(x)= \begin{cases}-1+k, & \left(4\left(n_{1}^{v}+n_{2}^{v}\right)+1\right) \delta / 4<x<\left(2\left(n_{1}^{v}+n_{2}^{v}\right)+1\right) \delta / 2-\varepsilon T^{\prime}, \\ v_{0}\left(\frac{\left(2\left(n_{1}^{v}+n_{2}^{v}\right)+1\right) \delta / 2-x}{\varepsilon}\right), & \left|x-\left(2\left(n_{1}^{v}+n_{2}^{v}\right)+1\right) \delta / 2\right| \leqslant \varepsilon T^{\prime}, \\ 1-k, & \left(2\left(n_{1}^{v}+n_{2}^{v}\right)+1\right) \delta / 2+\varepsilon T^{\prime}<x<\left(n_{1}^{v}+n_{2}^{v}+1\right) \delta-\varepsilon T, \\ v_{\varepsilon, 1}^{n_{1}^{v}+n_{2}^{v}+1}(x), & \left(n_{1}^{v}+n_{2}^{v}+1\right) \delta-\varepsilon T \leqslant x \leqslant\left(4\left(n_{1}^{v}+n_{2}^{v}\right)+5\right) \delta / 4 .\end{cases}
$$

Taking $u_{\varepsilon}^{v}:=\left.U_{\varepsilon}^{v}\right|_{(0,1)}$, we have

$$
\begin{aligned}
\limsup _{\varepsilon \rightarrow 0} F_{\varepsilon}^{k(1)}\left(u_{\varepsilon}^{v}\right) & \leqslant \lim _{\varepsilon \rightarrow 0}\left(\left(2 C_{1}^{k}+\eta\right)\left(n_{1}^{v}+n_{2}^{v}\right) \delta+\left(2 C_{2}^{k}+\eta\right) \delta\right)\left[\frac{1}{\left(n_{1}^{v}+n_{2}^{v}+1\right) \delta}\right] \\
& =\left(2 C_{1}^{k}+\eta\right) \frac{n_{1}^{v}+n_{2}^{v}}{n_{1}^{v}+n_{2}^{v}+1}+\left(2 C_{2}^{k}+\eta\right) \frac{1}{n_{1}^{v}+n_{2}^{v}+1}=: a^{k, v}
\end{aligned}
$$

Moreover,

$$
\lim _{\nu \rightarrow 0} a^{k, v}=2 C_{1}^{k}+\eta
$$

and a standard diagonalization argument yields a positive decreasing (as $\varepsilon$ decreases) function $v=$ $\nu(\varepsilon)$ such that $\nu(\varepsilon) \rightarrow 0$ as $\varepsilon \rightarrow 0$, for which

$$
\limsup _{\varepsilon \rightarrow 0} F_{\varepsilon}^{k(1)}\left(u_{\varepsilon}^{\nu(\varepsilon)}\right) \leqslant 2 C_{1}^{k}+\eta
$$

Finally, using 4.29) and the fact that $\varepsilon \ll \delta$ it is easy to check that we also have $u_{\varepsilon}^{\nu(\varepsilon)} \rightarrow c$ in $L^{2}(0,1)$, and hence the $\Gamma$-limsup for $-1<c<1$ and $k \leqslant 1 / 2$.

Let $k>1 / 2$; now to approximate a constant $c$, on one hand, it is not any more "optimal" to oscillate between $1+k, 1-k$ and $-1+k,-1-k$, because in this case the most convenient transition is the one from $1-k$ to $-1+k$ (see Remark 4.1). On the other hand, using convenient transitions (following the construction made for $c=1$ ) only permits us to approximate $c=0$. Then, for instance, to obtain a recovery sequence for $0<c<1$ it is necessary to mix, in the right proportion, oscillations between $1+k, 1-k$ with those between $1-k,-1+k$. In this way, following a procedure which is similar to that of the previous case, but now with

$$
\frac{n_{1}^{v}}{n_{2}^{v}} \rightarrow \frac{c}{1-c} \quad \text { as } v \rightarrow 0
$$

it is possible to construct a sequence $u_{\varepsilon} \rightarrow c$ in $L^{2}(0,1)$ such that

$$
\begin{aligned}
\limsup _{\varepsilon \rightarrow 0} F_{\varepsilon}^{k(1)}\left(u_{\varepsilon}\right) & \leqslant \lim _{\varepsilon \rightarrow 0}\left(\left(2 C_{1}^{k}+\eta\right)\left(n_{1}^{\nu(\varepsilon)}+1\right) \delta+\left(2 C_{2}^{k}+\eta\right) n_{2}^{\nu(\varepsilon)} \delta\right)\left[\frac{1}{\left(n_{1}^{\nu(\varepsilon)}+n_{2}^{\nu(\varepsilon)}+1\right) \delta}\right] \\
& =c\left(2 C_{1}^{k}+\eta\right)+(1-c)\left(2 C_{2}^{k}+\eta\right)=2\left(C_{1}^{k}-C_{2}^{k}\right) c+2 C_{2}^{k}+\eta .
\end{aligned}
$$

This concludes the proof of the $\Gamma$-limsup inequality. 


\subsection{Second-order $\Gamma$-limit}

In the spirit of studying the asymptotic behavior of the family of functionals $\left(F_{\varepsilon}^{k}\right)$, Theorem 4.2 suggests that the characterization of the limit points of sequences of minimizers, as well as the development for the minimum values $m_{\varepsilon}^{k}$, can be improved for $k \leqslant 1 / 2$. As for $k>1 / 2$, the functional $F^{k(1)}$ admits the unique minimizer $u \equiv 0$. Nonetheless, as we will show in Section 4.3.2. the nonstrict convexity of $\psi^{k}$ permits us to consider a further scaling, and thus another term in the $\Gamma$-development, in this case as well.

Since the two cases $k \leqslant 1 / 2, k>1 / 2$ need a different handling, we discuss the second-order asymptotic analysis for $\left(F_{\varepsilon}^{k}\right)$ in two separate sections. The first one, Section 4.3.1 is devoted to the case $k \leqslant 1 / 2$, which is also referred to as the case of small perturbations; while the second one, Section 4.3.2 deals with $k>1 / 2$, the case of large perturbations.

4.3.1 $k<1 / 2$ : small perturbations. In terms of the asymptotic development for the minimum value $m_{\varepsilon}^{k}$, the combined computation of the zero-order and the first-order $\Gamma$-limit gives

$$
m_{\varepsilon}^{k}=\frac{\varepsilon}{\delta} 2 C_{1}^{k}+o\left(\frac{\varepsilon}{\delta}\right) \quad \text { as } \varepsilon \rightarrow 0 .
$$

Then, to further improve the above development, we need to quantify the "small" error $o(\varepsilon / \delta)$, and hence to identify the next meaningful scaling that we denote by $\underline{\lambda}_{\infty}^{(2)}(\varepsilon)$ (not to be confused with the scaling for $k>1 / 2$, which we will denote by $\left.\bar{\lambda}_{\infty}^{(2)}(\varepsilon)\right)$.

Once $\underline{\lambda}_{\infty}^{(2)}(\varepsilon)$ is conjectured, we study the $\Gamma$-limit of the scaled functionals

$$
F_{\varepsilon}^{k(2)}:=\frac{F_{\varepsilon}^{k}-\frac{\varepsilon}{\delta} 2 C_{1}^{k}}{\underline{\lambda}_{\infty}^{(2)}(\varepsilon)} .
$$

To guess the second meaningful scale $\underline{\lambda}_{\infty}^{(2)}(\varepsilon)$, we first observe that in order to make $F_{\varepsilon}^{k}-\frac{\varepsilon}{\delta} 2 C_{1}^{k}$ vanish, a sequence must oscillate (except possibly on a finite number of $\delta$-intervals) between $1+k$, $1-k$ or between $-1+k,-1-k$. Hence, we focus on a $\delta / 2$-interval, for instance $(\delta / 4,3 \delta / 4)$, and we estimate the contribution of $F_{\varepsilon}^{k}-\frac{\varepsilon}{\delta} 2 C_{1}^{k}$ over this interval. We have

$$
\begin{aligned}
\int_{\delta / 4}^{3 \delta / 4}\left(W^{k}\left(\frac{x}{\delta}, u\right)+\varepsilon^{2}\left(u^{\prime}\right)^{2}\right) \mathrm{d} x-\varepsilon C_{1}^{k} \\
=\varepsilon\left(\int_{\delta / 4}^{3 \delta / 4}\left(\frac{1}{\varepsilon} W^{k}\left(\frac{x}{\delta}, u\right)+\varepsilon\left(u^{\prime}\right)^{2}\right) \mathrm{d} x-C_{1}^{k}\right) \\
=\varepsilon\left(\int_{1 / 4}^{1 / 2}\left(\frac{\delta}{\varepsilon} W_{1}^{k}(v)+\frac{\varepsilon}{\delta}\left(v^{\prime}\right)^{2}\right) \mathrm{d} x+\int_{1 / 2}^{3 / 4}\left(\frac{\delta}{\varepsilon} W_{2}^{k}(v)+\frac{\varepsilon}{\delta}\left(v^{\prime}\right)^{2}\right) \mathrm{d} x-C_{1}^{k}\right)
\end{aligned}
$$

with $v(x):=u(\delta x)$. A direct minimization of (4.30) yields

$$
{ }_{\varepsilon} C_{1}^{k}\left(\tanh \left(\frac{\delta}{4 \varepsilon}\right)-1\right)=O\left(\varepsilon e^{-\delta / 2 \varepsilon}\right) \quad \text { as } \varepsilon \rightarrow 0,
$$

and it is easy to check that the above minimum value is attained, for instance, at the function $v(x):=$ $v_{\varepsilon}^{1}(1 / 2-x / \delta)$ (with $v_{\varepsilon}^{1}$ defined as in 4.12 of Proposition 4.5. with $\eta=\varepsilon / \delta$ ). Thus, by repeating 
the previous argument over each $\delta / 2$-interval (except possibly a finite number of them) we get a first energy contribution of order $(\varepsilon / \delta) e^{-\delta / 2 \varepsilon}$. The energy 4.30 is minimized also by $v(x)-2$ (i.e., by a transition with average -1 ), hence the total energy of a minimizing sequence may well be the result of a finite number of transitions from oscillations with average 1 to oscillations with average -1 and vice versa. Since each of these transitions between the "oscillating phases" \pm 1 gives an additional contribution of order $\varepsilon$, the total energy contribution of a minimizing sequence turns out to be of order

$$
\frac{\varepsilon}{\delta} e^{-\delta / 2 \varepsilon}+\varepsilon
$$

Then $\underline{\lambda}_{\infty}^{(2)}(\varepsilon)=(\varepsilon / \delta) e^{-\delta / 2 \varepsilon}$ if we have

$$
\frac{\varepsilon}{\delta} e^{-\delta / 2 \varepsilon} \gg \varepsilon, \quad \text { i.e., } \quad e^{-\delta / 2 \varepsilon} \gg \delta .
$$

Loosely speaking, when this scale is relevant, we have to consider first the error that we make by "cutting the tails" of the $1 / \delta$ infinite transitions that we are gluing together. Thus, in this case we expect to find again a constant $\Gamma$-limit which is now given by

$$
\lim _{\varepsilon \rightarrow 0} \frac{2 C_{1}^{k}(\tanh (\delta / 4 \varepsilon)-1)}{e^{-\delta / 2 \varepsilon}}=-4 C_{1}^{k}
$$

We instead get $\underline{\lambda}_{\infty}^{(2)}(\varepsilon)=\varepsilon$ if we have

$$
e^{-\delta / 2 \varepsilon} \ll \delta .
$$

This choice penalizes the passages from the oscillations "around 1" to those "around -1 " and vice versa. Therefore, if $\underline{\lambda}_{\infty}^{(2)}(\varepsilon)=\varepsilon$ we expect that $\left(F_{\varepsilon}^{k(2)}\right) \Gamma$-converges to a surface energy which penalizes the jumps between 1 and -1 of the limit configuration. It is worth pointing out that assumption 4.31 covers the cases $\delta=\varepsilon^{\alpha}$ for all $\alpha<1$, and hence seems more natural.

Since we are concerned not only with a better development for $m_{\varepsilon}^{k}$ but also with an improvement in the characterization of the asymptotic behavior of sequences of minimizers, we decide to focus on the case $e^{-\delta / 2 \varepsilon} \ll \delta$, and hence on the case

$$
\underline{\lambda}_{\infty}^{(2)}(\varepsilon)=\varepsilon .
$$

Then we look at the scaled functionals

$$
\begin{aligned}
F_{\varepsilon}^{k(2)}(u) & =\frac{1}{\varepsilon} F_{\varepsilon}^{k}(u)-\frac{1}{\delta} 2 C_{1}^{k} \\
& = \begin{cases}\int_{0}^{1}\left(\frac{1}{\varepsilon} W^{k}\left(\frac{x}{\delta}, u\right)+\varepsilon\left(u^{\prime}\right)^{2}\right) \mathrm{d} x-\frac{2 C_{1}^{k}}{\delta} & \text { if } u \in W^{1,2}(0,1), \\
+\infty & \text { otherwise. }\end{cases}
\end{aligned}
$$

We now come to a rigorous justification of what has only been heuristically conjectured.

We start by proving that the uniform boundedness of $F_{\varepsilon}^{k(2)}\left(u_{\varepsilon}\right)$ implies, for the limit configuration $u$, both the constraint $u(x) \in\{ \pm 1\}$ a.e. and that $u$ is piecewise constant.

LEMmA 4.6 If $\sup _{\varepsilon} F_{\varepsilon}^{k(2)}\left(u_{\varepsilon}\right)<+\infty$ then, up to taking a subsequence, $\left(u_{\varepsilon}\right)$ converges to some function $u \in B V((0,1) ;\{ \pm 1\})$ with respect to weak $L^{2}$-convergence. 
Proof. For fixed $\varepsilon>0$, starting at 0 , we partition [0,1] into subintervals $I_{i}^{\delta}, i=1, \ldots,[1 / \delta]$, of length $\delta$ (except possibly the last of length less than $\delta$ ).

Let $u_{\varepsilon}$ be such that $\sup _{\varepsilon} F_{\varepsilon}^{k(2)}\left(u_{\varepsilon}\right)<+\infty$ and set $u_{\delta}^{ \pm}(x):=u^{ \pm}(x / \delta)$, where $u^{-}, u^{+}$are the 1 -periodic functions on $\mathbb{R}^{+}$, defined on $(0,1)$ by

$$
u^{-}(t):=\left\{\begin{array}{ll}
-1+k & \text { if } t \in(0,1 / 2), \\
-1-k & \text { if } t \in(1 / 2,1),
\end{array} \quad u^{+}(t):= \begin{cases}1+k & \text { if } t \in(0,1 / 2) \\
1-k & \text { if } t \in(1 / 2,1)\end{cases}\right.
$$

The first step of the proof consists in showing that for any fixed $\eta>0$, if $\mathcal{I}_{\eta}^{\delta}$ is the set of all indices $i$ in $\{1, \ldots,[1 / \delta]\}$ such that

$$
\min \left\{f_{I_{i}^{\delta}}\left|u_{\varepsilon}-u_{\delta}^{-}\right| \mathrm{d} x, f_{I_{i}^{\delta}}\left|u_{\varepsilon}-u_{\delta}^{+}\right| \mathrm{d} x\right\} \leqslant \eta
$$

then

$$
\lim _{\varepsilon \rightarrow 0} \delta \#\left(\mathcal{I}_{\eta}^{\delta}\right)=1
$$

In other words, for every $\eta>0, \sqrt{4.34}$ is satisfied on a "large" number of intervals $I_{i}^{\delta}$ (provided that $\varepsilon$ is sufficiently small). In order to prove 4.35 , we estimate the cardinality of the family $\mathcal{J}_{\eta}^{\delta}$ of indices $i$ for which

$$
\min \left\{f_{I_{i}^{\delta}}\left|u_{\varepsilon}-u_{\delta}^{-}\right| \mathrm{d} x, f_{I_{i}^{\delta}}\left|u_{\varepsilon}-u_{\delta}^{+}\right| \mathrm{d} x\right\}>\eta .
$$

Before starting our computation, we notice that the statement

$$
\text { there exists } M>0 \text { such that }\left|u_{\varepsilon}(x)\right| \leqslant M \text { for all } x \in I_{i}^{\delta}
$$

holds true, with the same constant $M$ (e.g., $M=2$ ), except for at most a bounded number of indices $i$. In fact, arguing as in the proof of Proposition 4.5 (ii), one can easily deduce the above statement from the uniform boundedness of $F_{\varepsilon}^{k(2)}\left(u_{\varepsilon}\right)$. So from now on, we focus on those intervals $I_{i}^{\delta}$ in which 4.36 is satisfied.

$$
\begin{aligned}
& \text { If } i \in \mathcal{J}_{\eta}^{\delta} \text { then } \\
& \eta<f_{I_{i}^{\delta}}\left|u_{\varepsilon}-u_{\delta}^{+}\right| \mathrm{d} x=\frac{1}{\delta} \int_{\left\{x \in I_{i}^{\delta}:\left|u_{\varepsilon}-u_{\delta}^{+}\right| \leqslant \eta / 2\right\}}\left|u_{\varepsilon}-u_{\delta}^{+}\right| \mathrm{d} x+\frac{1}{\delta} \int_{\left\{x \in I_{i}^{\delta}:\left|u_{\varepsilon}-u_{\delta}^{+}\right|>\eta / 2\right\}}\left|u_{\varepsilon}-u_{\delta}^{+}\right| \mathrm{d} x \\
& \leqslant \frac{\eta}{2}+\frac{c(M)}{\delta}\left|\left\{x \in I_{i}^{\delta}:\left|u_{\varepsilon}-u_{\delta}^{+}\right|>\frac{\eta}{2}\right\}\right| \text {, }
\end{aligned}
$$

hence

$$
\left|\left\{x \in I_{i}^{\delta}:\left|u_{\varepsilon}-u_{\delta}^{+}\right|>\frac{\eta}{2}\right\}\right|>c(M, \eta) \delta .
$$

The same conclusion also holds with $u_{\delta}^{-}$replacing $u_{\delta}^{+}$. As a consequence,

$$
\int_{I_{i}^{\delta}} W^{k}\left(\frac{x}{\delta}, u_{\varepsilon}\right) \mathrm{d} x>C \delta \quad \text { for every } i \in \mathcal{J}_{\eta}^{\delta},
$$

and this implies

$$
F_{\varepsilon}^{k}\left(u_{\varepsilon}\right) \geqslant \#\left(\mathcal{J}_{\eta}^{\delta}\right) C \delta .
$$


By hypothesis $F_{\varepsilon}^{k(2)}\left(u_{\varepsilon}\right) \leqslant C$, and therefore

$$
F_{\varepsilon}^{k}\left(u_{\varepsilon}\right) \leqslant \varepsilon C+\frac{\varepsilon}{\delta} 2 C_{1}^{k}=O\left(\frac{\varepsilon}{\delta}\right) \quad \text { as } \varepsilon \rightarrow 0 .
$$

Combining (4.37) and (4.38) we get

$$
\delta \#\left(\mathcal{J}_{\eta}^{\delta}\right) \rightarrow 0 \quad \text { as } \varepsilon \rightarrow 0,
$$

and hence the desired result follows.

Let $N_{\varepsilon}$ be the overall number of transitions of $u_{\varepsilon}$ between $1+k \pm \eta$ and $-1-k \pm \eta ; 1+k \pm \eta$ and $-1+k \pm \eta ; 1-k \pm \eta$ and $-1-k \pm \eta$; and $1-k \pm \eta$ and $-1+k \pm \eta$. From now on we refer to these transitions as the expensive transitions. To conclude the proof we notice that the most convenient of these transitions is the one from $-1+k+\eta$ to $1-k-\eta$ and, in terms of $F_{\varepsilon}^{k(0)}$, it costs at least $\varepsilon\left(C_{2}^{k}-C \eta^{2}\right)$. Now, recalling that $C_{2}^{k}>C_{1}^{k}$, for sufficiently small $\eta$ we have $C_{2}^{k} \geqslant C_{1}^{k}-C \eta^{2}$, and thus from the uniform boundedness of $F_{\varepsilon}^{k(2)}\left(u_{\varepsilon}\right)$ we deduce that $N_{\varepsilon} \leqslant \bar{N}$ for some $\bar{N} \in \mathbb{N}$. As a consequence, (a subsequence of) $u_{\varepsilon}$ makes a number of expensive transitions which is actually independent of $\varepsilon$; we denote this number by $N$. Let $S_{\varepsilon}=\left\{t_{1}^{\varepsilon}, \ldots, t_{N-1}^{\varepsilon}\right\}$ (with $\left.t_{n}^{\varepsilon}<t_{n+1}^{\varepsilon}, n=1, \ldots, N-2\right)$ be a set of points dividing $(0,1)$ into $N$ subintervals each containing only one expensive transition for $u_{\varepsilon}$. Passing to further subsequences if necessary, we can suppose that

$$
t_{n}^{\varepsilon} \rightarrow t_{n} \quad \text { as } \varepsilon \rightarrow 0 \quad \text { for } n=1, \ldots, N-1 .
$$

Then, for fixed $\sigma>0$, if we consider the $N$ intervals

$$
J_{\sigma}^{n}=\left(t_{n}+\sigma, t_{n+1}-\sigma\right), \quad n=0, \ldots, N-1 \quad\left(\text { with } t_{0}=0, t_{N}=1\right)
$$

we see that

$$
J_{\sigma}^{n} \cap S_{\varepsilon}=\emptyset
$$

for sufficiently small $\varepsilon$ and for every $n=0, \ldots, N-1$. By virtue of 4.39 , applying to $J_{\sigma}^{n}$ the result established in the first part of the proof, we find that, for instance,

$$
\limsup _{\varepsilon \rightarrow 0} \int_{J_{\sigma}^{n}}\left|u_{\varepsilon}-u_{\delta}^{+}\right| \mathrm{d} x \leqslant C \eta .
$$

On the other hand, by weak compactness we have $u_{\varepsilon} \rightarrow u$ in $L^{2}\left(J_{\sigma}^{n}\right)$, while $u_{\delta}^{+} \rightarrow 1$ in $L^{2}\left(J_{\sigma}^{n}\right)$ from 4.33 ; thus by the weak lower semicontinuity of the $L^{1}$-norm we deduce

$$
\int_{J_{\sigma}^{n}}|u-1| \mathrm{d} x \leqslant \liminf _{\varepsilon \rightarrow 0} \int_{J_{\sigma}^{n}}\left|u_{\varepsilon}-u_{\delta}^{+}\right| \mathrm{d} x,
$$

and combining this with 4.40 we find

$$
\int_{J_{\sigma}^{n}}|u-1| \mathrm{d} x \leqslant C \eta \quad \text { for all } \eta, \sigma>0 .
$$

Finally, by the arbitrariness of $\eta$ and $\sigma$ it follows that $u \equiv 1$ on $J^{n}=\left(t_{n}, t_{n+1}\right)$. Thus by repeating the above argument on all intervals $J^{n}(n=0, \ldots, N-1)$, which form a partition of [0, 1], we get the conclusion.

In the remainder of this section, we work under the additional assumption $1 / \delta \in \mathbb{N}$. This assumption will in some cases be essential, as it permits us not to consider the effects due to boundary mismatch, while, at other points, it will provide only some technical help. 
THEOREM 4.7 Let $\delta$ be such that $\delta \gg e^{-\delta / 2 \varepsilon}$ and $1 / \delta \in \mathbb{N}$. The family of functionals $F_{\varepsilon}^{k(2)}$ defined in 4.32 $\Gamma$-converges with respect to weak $L^{2}$-convergence to the functional defined on $L^{2}(0,1)$ by

$$
F^{k(2)}(u)= \begin{cases}\left(C_{2}^{k}-C_{1}^{k}\right) \#(S(u))-C_{1}^{k} & \text { if } u \in B V((0,1) ;\{ \pm 1\}) \\ +\infty & \text { otherwise }\end{cases}
$$

Proof. Step 1: $\Gamma$-liminf inequality. We have to prove that if $u_{\varepsilon} \rightarrow u$ in $L^{2}(0,1)$ and $\sup _{\varepsilon} F_{\varepsilon}^{k(2)}\left(u_{\varepsilon}\right)<+\infty$, then $F^{k(2)}(u) \leqslant \liminf _{\varepsilon \rightarrow 0} F_{\varepsilon}^{k(2)}\left(u_{\varepsilon}\right)$.

By Lemma 4.6 we already know that $u \in B V((0,1) ;\{ \pm 1\})$; set $N:=\#(S(u))$. For fixed $\varepsilon>0$, we consider the partition of $(\delta / 4,1-\delta / 4)$ into subintervals $I_{i}^{\delta}:=((2 i-1) \delta / 4,(2 i+1) \delta / 4)$ with $i=1, \ldots, 2 / \delta-1$ and we rewrite $F_{\varepsilon}^{k(2)}\left(u_{\varepsilon}\right)$ as

$$
\begin{aligned}
F_{\varepsilon}^{k(2)}\left(u_{\varepsilon}\right)= & \int_{0}^{\delta / 4}\left(\frac{1}{\varepsilon} W_{1}^{k}\left(u_{\varepsilon}\right)+\varepsilon\left(u_{\varepsilon}^{\prime}\right)^{2}\right) \mathrm{d} x+\sum_{i=1}^{2 / \delta-1}\left(\frac{1}{\varepsilon} F_{\varepsilon}^{k}\left(u_{\varepsilon} ; I_{i}^{\delta}\right)-C_{1}^{k}\right) \\
& +\int_{1-\delta / 4}^{1}\left(\frac{1}{\varepsilon} W_{2}^{k}\left(u_{\varepsilon}\right)+\varepsilon\left(u_{\varepsilon}^{\prime}\right)^{2}\right) \mathrm{d} x-C_{1}^{k},
\end{aligned}
$$

where

$$
F_{\varepsilon}^{k}\left(u_{\varepsilon} ; I_{i}^{\delta}\right):=\int_{(2 i-1) \delta / 4}^{(2 i+1) \delta / 4}\left(W^{k}\left(\frac{x}{\delta}, u_{\varepsilon}\right)+\varepsilon^{2}\left(u_{\varepsilon}^{\prime}\right)^{2}\right) \mathrm{d} x .
$$

By a straightforward calculation we find that

$$
\min _{v \in W^{1,2}\left(I_{i}^{\delta}\right)}\left(\frac{1}{\varepsilon} F_{\varepsilon}^{k}\left(v ; I_{i}^{\delta}\right)-C_{1}^{k}\right)=C_{1}^{k}\left(\tanh \left(\frac{\delta}{4 \varepsilon}\right)-1\right)=O\left(e^{-\delta / 2 \varepsilon}\right) \quad \text { as } \varepsilon \rightarrow 0
$$

for every $i=1, \ldots, 2 / \delta-1$, and the minimum is attained at

$$
u_{\varepsilon, 1}^{i}(x)=\left\{\begin{array}{ll}
v_{\varepsilon}^{1}(i / 2-x / \delta) & \text { if } i \text { is odd } \\
v_{\varepsilon}^{1}(x / \delta-i / 2) & \text { if } i \text { is even }
\end{array} \quad \text { for } x \in I_{i}^{\delta}, i=1, \ldots, 2 / \delta-1,\right.
$$

where $v_{\varepsilon}^{1}$ is as in 4.13 with $\eta=\varepsilon / \delta$.

If $N=0$, since

$$
F_{\varepsilon}^{k(2)}\left(u_{\varepsilon}\right) \geqslant \sum_{i=1}^{2 / \delta-1}\left(\frac{1}{\varepsilon} F_{\varepsilon}^{k(0)}\left(u_{\varepsilon} ; I_{i}^{\delta}\right)-C_{1}^{k}\right)-C_{1}^{k}
$$

we obtain the assertion by simply taking the minimum of each term on the right hand side of (4.42) and recalling that by hypothesis $\lim _{\varepsilon \rightarrow 0} e^{-\delta / 2 \varepsilon} / \delta=0$. If $N>0$, let $N_{\varepsilon}$ be as in Lemma 4.6 Then, as already observed, $N_{\varepsilon}$ is bounded and moreover

$$
\liminf _{\varepsilon \rightarrow 0} N_{\varepsilon} \geqslant N
$$

To get the liminf inequality for the $\Gamma$-limit we need a lower bound for the energy of the expensive transitions. We first estimate the measure of the set where a transition between two zeroes of $W^{k}$ may occur. Let $\eta$ be a positive number and set

$$
J_{i}^{\delta}:=\left\{t \in I_{i}^{\delta}: \operatorname{dist}\left(u_{\varepsilon}, Z_{i}^{k, \delta}(t)\right)>\eta\right\},
$$


where

$$
Z_{i}^{k, \delta}(t):=\left\{\begin{array}{ll}
Z_{1}^{k} & \text { if } t \in((2 i-1) \delta / 4, i \delta / 2) \\
Z_{2}^{k} & \text { if } t \in(i \delta / 2,(2 i+1) \delta / 4)
\end{array} \quad \text { if } i\right. \text { is odd }
$$

while

$$
Z_{i}^{k, \delta}(t):=\left\{\begin{array}{ll}
Z_{2}^{k} & \text { if } t \in((2 i-1) \delta / 4, i \delta / 2) \\
Z_{1}^{k} & \text { if } t \in(i \delta / 2,(2 i+1) \delta / 4)
\end{array} \quad \text { if } i\right. \text { is even }
$$

We have

$$
\frac{1}{\varepsilon} F_{\varepsilon}^{k}\left(u_{\varepsilon} ; I_{i}^{\delta}\right) \geqslant \frac{1}{\varepsilon} F_{\varepsilon}^{k}\left(u_{\varepsilon} ; J_{i}^{\delta}\right) \geqslant C \eta^{2} \frac{\left|J_{i}^{\delta}\right|}{\varepsilon}
$$

and from $\sup _{\varepsilon} F_{\varepsilon}^{k(2)}\left(u_{\varepsilon}\right)<+\infty$ we deduce that, for every $i,\left|J_{i}^{\delta}\right|=O(\varepsilon)$ as $\varepsilon$ tends to zero. Hence we can conclude that an expensive transition may only be of two different types.

Type 1: the transition entirely occurs in an interval $I_{i_{0}}^{\delta}$ for some $i_{0}$; in this case we have

$$
\frac{1}{\varepsilon} F_{\varepsilon}^{k}\left(u_{\varepsilon} ; I_{i_{0}}^{\delta}\right) \geqslant C_{W^{k}}(1-k-\eta,-1+k+\eta) \geqslant C_{2}^{k}-C \eta^{2} .
$$

Type 2: the transition occurs between two adjacent intervals $I_{i_{0}}^{\delta}, I_{i_{0}+1}^{\delta}$ for some $i_{0}$; in this case we have

$$
\begin{aligned}
\frac{1}{\varepsilon} F_{\varepsilon}^{k}\left(u_{\varepsilon} ; I_{i_{0}}^{\delta}\right)+\frac{1}{\varepsilon} F_{\varepsilon}^{k}\left(u_{\varepsilon} ; I_{i_{0}+1}^{\delta}\right) & \geqslant C_{W_{1}^{k}}(1+k-\eta,-1+k+\eta)\left(=C_{W_{2}^{k}}(1-k-\eta,-1-k+\eta)\right) \\
& \geqslant C_{W}-C \eta^{2} .
\end{aligned}
$$

So if we let $N_{\varepsilon}^{j}(j=1,2)$ denote the number of expensive transitions of type $j$, then $N_{\varepsilon}=N_{\varepsilon}^{1}+N_{\varepsilon}^{2}$. By combining (4.44) and 4.45) we find that

$$
\begin{aligned}
F_{\varepsilon}^{k(2)}\left(u_{\varepsilon}\right) \geqslant & \left(\frac{2}{\delta}-1-N_{\varepsilon}^{1}-2 N_{\varepsilon}^{2}\right) C_{1}^{k}\left(\tanh \left(\frac{\delta}{4 \varepsilon}\right)-1\right) \\
& +N_{\varepsilon}^{1}\left(C_{2}^{k}-C_{1}^{k}-C \eta^{2}\right)+N_{\varepsilon}^{2}\left(C_{W}-2 C_{1}^{k}-C \eta^{2}\right)-C_{1}^{k} \\
\geqslant & \frac{2}{\delta} C_{1}^{k}\left(\tanh \left(\frac{\delta}{4 \varepsilon}\right)-1\right)+N_{\varepsilon}\left(C_{2}^{k}-C_{1}^{k}-C \eta^{2}\right)-C_{1}^{k},
\end{aligned}
$$

using the fact that $C_{W}=2$ and $2 \geqslant C_{1}^{k}+C_{2}^{k}$ in the last inequality. Finally, passing to the liminf, in view of 4.43 we get

$$
\liminf _{\varepsilon \rightarrow 0} F_{\varepsilon}^{k(2)}\left(u_{\varepsilon}\right) \geqslant N\left(C_{2}^{k}-C_{1}^{k}-C \eta^{2}\right)-C_{1}^{k} \quad \text { for all } \eta>0
$$

and thus letting $\eta$ go to zero yields the $\Gamma$-liminf inequality.

Step 2: $\Gamma$-limsup inequality. Let $x_{0} \in(0,1)$. To check the limsup inequality, it will suffice to deal with the case

$$
u(x)= \begin{cases}-1 & \text { if } x<x_{0} \\ 1 & \text { if } x \geqslant x_{0}\end{cases}
$$

Let $u_{\varepsilon, 1}^{i}$ be as in 4.41 and set $u_{\varepsilon,-1}^{i}:=u_{\varepsilon, 1}^{i}-2$ for $i=1, \ldots, 2 / \delta-1$. As a recovery sequence we take 
$u_{\varepsilon}(x)= \begin{cases}u_{\varepsilon, 1}^{1}(\delta / 4) & \text { if } x \in(0, \delta / 4), \\ u_{\varepsilon, 1}^{i}(x) & \text { if } x \in((2 i-1) \delta / 4,(2 i+1) \delta / 4) \text { for } i=1, \ldots, 2\left[x_{0} / \delta\right]-2, \\ \hat{w}_{\varepsilon}(x) & \text { if } x \in\left(\left(4\left[x_{0} / \delta\right]-3\right) \delta / 4,\left(4\left[x_{0} / \delta\right]+3\right) \delta / 4\right), \\ u_{\varepsilon,-1}^{i}(x) & \text { if } x \in((2 i-1) \delta / 4,(2 i+1) \delta / 4) \text { for } i=2\left[x_{0} / \delta\right]+2, \ldots, 2 / \delta-1, \\ u_{\varepsilon,-1}^{2 / \delta-1}(1-\delta / 4) & \text { if } x \in(1-\delta / 4,1),\end{cases}$

with

$$
\hat{w}_{\varepsilon}(x)= \begin{cases}u_{\varepsilon, 1}^{2\left[x_{0} / \delta\right]-1}(x) & \text { if }\left(4\left[x_{0} / \delta\right]-3\right) \delta / 4<x \leqslant\left(4\left[x_{0} / \delta\right]-1\right) \delta / 4-\varepsilon, \\ l_{\varepsilon}(x) & \text { if }\left(4\left[x_{0} / \delta\right]-1\right) \delta / 4-\varepsilon<x<\left(4\left[x_{0} / \delta\right]-1\right) \delta / 4+\varepsilon, \\ v_{\varepsilon}^{0}\left(x / \delta-\left[x_{0} / \delta\right]\right) & \text { if }\left(4\left[x_{0} / \delta\right]-1\right) \delta / 4+\varepsilon \leqslant x \leqslant\left(4\left[x_{0} / \delta\right]+1\right) \delta / 4-\varepsilon, \\ l_{\varepsilon}(x-\delta / 2)-2 & \text { if }\left(4\left[x_{0} / \delta\right]+1\right) \delta / 4-\varepsilon<x \leqslant\left(4\left[x_{0} / \delta\right]+1\right) \delta / 4+\varepsilon, \\ u_{\varepsilon,-1}^{2\left[x_{0} / \delta\right]+1}(x) & \text { if }\left(4\left[x_{0} / \delta\right]+1\right) \delta / 4+\varepsilon<x<\left(4\left[x_{0} / \delta\right]+3\right) \delta / 4,\end{cases}
$$

where $v_{\varepsilon}^{0}, v_{\varepsilon}^{1}$ are as in 4.12 and 4.13 respectively and $l_{\varepsilon}$ is the linear function defined by

$$
l_{\varepsilon}(x):=\frac{v_{\varepsilon}^{0}(\varepsilon / \delta-1 / 4)-v_{\varepsilon}^{1}(\varepsilon / \delta-1 / 4)}{2 \varepsilon}\left(x-\left(4\left[\frac{x_{0}}{\delta}\right]-1\right) \frac{\delta}{4}+\varepsilon\right)+v_{\varepsilon}^{0}\left(\frac{\varepsilon}{\delta}-\frac{1}{4}\right) .
$$

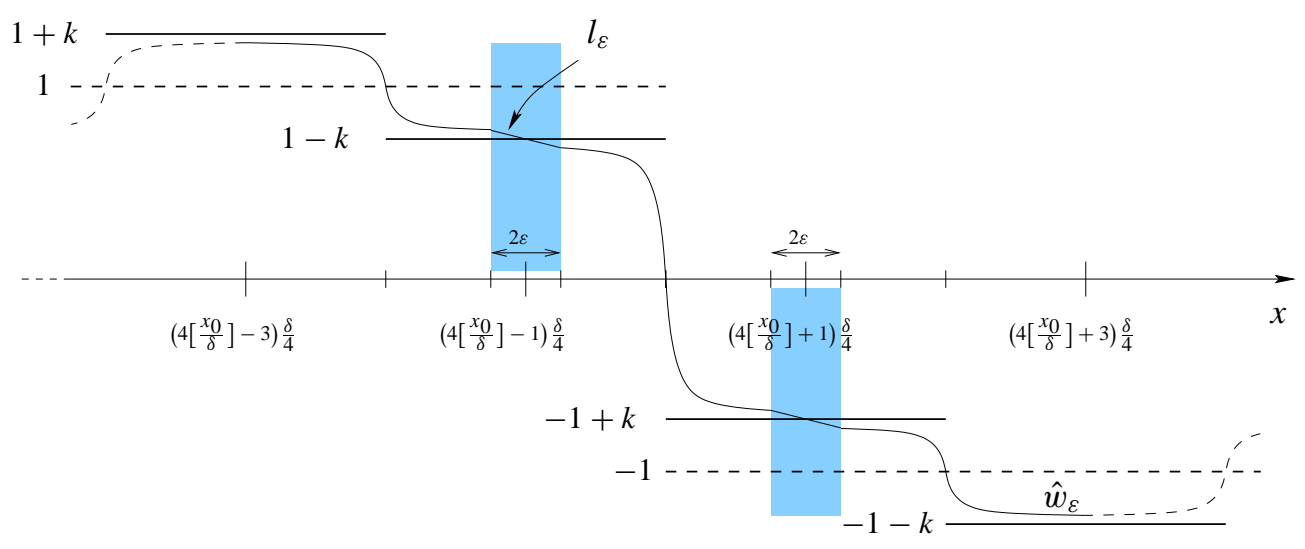

FIG. 11. The joining transition $\hat{w}_{\mathcal{E}}$.

In fact, it is easy to check that $u_{\varepsilon} \rightarrow u$ in $L^{2}(0,1)$ and that the energy contribution due to the linear, joining function $l_{\varepsilon}$ is of order $e^{-\delta / 2 \varepsilon}$. Then

$$
\begin{aligned}
\limsup _{\varepsilon \rightarrow 0} F_{\varepsilon}^{k(2)} & \left(u_{\varepsilon}\right)=\limsup _{\varepsilon \rightarrow 0}\left(\int_{\delta / 4}^{1-\delta / 4}\left(\frac{1}{\varepsilon} W^{k}\left(\frac{x}{\delta}, u_{\varepsilon}\right)+\varepsilon\left(u_{\varepsilon}^{\prime}\right)^{2}\right) \mathrm{d} x-\frac{2 C_{1}^{k}}{\delta}\right) \\
& \leqslant \limsup _{\varepsilon \rightarrow 0}\left(\left(\frac{2}{\delta}-4\right) C_{1}^{k} \tanh \left(\frac{\delta}{4 \varepsilon}\right)+2 C_{1}^{k} \tanh \left(\frac{\delta}{4 \varepsilon}\right)+C_{2}^{k} \tanh \left(\frac{\delta}{4 \varepsilon}\right)-\frac{2 C_{1}^{k}}{\delta}\right) \\
& =\left(C_{2}^{k}-C_{1}^{k}\right)-C_{1}^{k}=F^{k(2)}(u),
\end{aligned}
$$

and this completes the proof. 
REMARK 4.8 In view of Theorem 4.7, in this case, $\varepsilon$ is not only the scale of macroscopic phase transitions but also that of external boundary layers. In the latter case the negative sign is explained by a missing (half) transition of the recovery sequence in the intervals $(0, \delta / 4)$ and $(1-\delta / 4,1)$.

The $\Gamma$-convergence results stated in Theorems 3.1, 4.2 and 4.7 are formally summarized by the $\Gamma$-development

$$
\begin{aligned}
F_{\varepsilon}^{k}(u) \stackrel{\Gamma}{=} & \int_{0}^{1} W^{* *}(u) \mathrm{d} x+\frac{\varepsilon}{\delta} 2 C_{1}^{k}+\varepsilon\left(\left(C_{2}^{k}-C_{1}^{k}\right) \#(S(u))-C_{1}^{k}\right)-\frac{\varepsilon}{\delta} e^{-\delta / 2 \varepsilon} 4 C_{1}^{k} \\
& +O\left(\varepsilon e^{-\delta / 2 \varepsilon}\right) .
\end{aligned}
$$

4.3.2 $k>1 / 2$ : large perturbations. For $k>1 / 2$ Theorem 4.2 states that $F_{\varepsilon}^{k(1)} \stackrel{\Gamma}{\longrightarrow} F^{k(1)}$ where

$$
F^{k(1)}(u)=\int_{0}^{1} \psi^{k}(u) \mathrm{d} x
$$

with $\psi^{k}(s)=2\left(C_{1}^{k}-C_{2}^{k}\right)|s|+2 C_{2}^{k}$ for $|s| \leqslant 1$. In this case, $\min _{|s| \leqslant 1} \psi^{k}(s)=\psi^{k}(0)=2 C_{2}^{k}$ and $F^{k(1)}$ has the unique minimizer $u=0$. As is customary, to obtain a nontrivial behavior we add the constraint

$$
\int_{0}^{1} v_{\varepsilon}=d
$$

with $d \neq 0$; to fix ideas, let $d \in(0,1)$.

REMARK 4.9 The zero-order and first-order $\Gamma$-limits for the Modica-Mortola functionals are stable by adding the "volume" constraint 4.47) (see [8], and [3, Proposition 6.6, Theorem 6.7] for the one-dimensional case). In our case, since we are dealing with a variant of the ModicaMortola model and with the different scaling $\varepsilon / \delta$, and since moreover integral constraints may not be automatically compatible with the refinement process of the computation of higher-order $\Gamma$-limits, we actually need to prove that (under some additional hypotheses) the $\Gamma$-convergence result stated in Theorem 4.2 is compatible with the integral constraint. We notice that since the equality (4.47) is closed for the weak $L^{2}$-convergence, the liminf inequality is trivial. To check the limsup inequality it again suffices to deal with piecewise constant functions (satisfying (4.47)). For simplicity we only detail the case of the constant target function $u=d$.

Let $\left(u_{\varepsilon}\right)$ be a sequence mixing oscillations "around 1" with oscillations "around 0 " as in Theorem 4.2. Step 2. Then, setting $d_{\varepsilon}:=\int_{0}^{1} u_{\varepsilon} \mathrm{d} x$, we have

$$
d_{\varepsilon}=n_{\varepsilon}^{1} \frac{\delta}{2}\left(1+O\left(\frac{\varepsilon}{\delta}\right)\right)+n_{\varepsilon}^{0} \frac{\delta}{2} O\left(\frac{\varepsilon}{\delta}\right) \quad \text { with } \quad 0 \leqslant n_{\varepsilon}^{0}, n_{\varepsilon}^{1} \leqslant \frac{2}{\delta}, n_{\varepsilon}^{0}+n_{\varepsilon}^{1}=\frac{2}{\delta},
$$

where, for fixed $\varepsilon>0, n_{\varepsilon}^{1}$ and $n_{\varepsilon}^{0}$ are the numbers of transitions of $u_{\varepsilon}$ between $1+k, 1-k$ and between $1-k,-1+k$, respectively. Hence if we let $n_{\varepsilon}^{1}$ vary from 0 to $2 / \delta$, then $d_{\varepsilon}$ goes from $d_{\varepsilon} \simeq 0$ to $d_{\varepsilon} \simeq 1$ (for $\varepsilon$ small). Moreover, the difference between two values of $d_{\varepsilon}$ corresponding to two consecutive values of $n_{\varepsilon}^{1}$ is of order $\delta$. Thus, we may choose $n_{\varepsilon}^{0}, n_{\varepsilon}^{1}$ in such a way that $u_{\varepsilon}$ is a recovery sequence for $d$, and we have

$$
\left|d-d_{\varepsilon}\right| \leqslant O(\delta) \quad \text { as } \varepsilon \rightarrow 0 .
$$


Now starting from $u_{\varepsilon}$ we want to construct a sequence $\left(v_{\varepsilon}\right)$ such that

$$
v_{\varepsilon} \rightarrow d \quad \text { in } \quad L^{2}(0,1), \quad \int_{0}^{1} v_{\varepsilon} \mathrm{d} x=d \quad \text { and } \quad F_{\varepsilon}^{k(1)}\left(v_{\varepsilon}\right) \rightarrow \int_{0}^{1} \psi^{k}(u) \mathrm{d} x .
$$

To this end, we focus on a $\delta / 2$-interval of type $((2 i-1) \delta / 4,(2 i+1) \delta / 4)$ with $i$ odd (the case of $i$ even can be treated similarly) and we suppose that on this interval $u_{\varepsilon}=v_{\varepsilon, 1}^{i}$, where $v_{\varepsilon, 1}^{i}$ is as in (4.28). Passing to a subsequence we can always assume that $d-d_{\varepsilon}$ has a constant sign, say $d_{\varepsilon} \leqslant d$. Then we define $v_{\varepsilon}$ on the interval $((2 i-1) \delta / 4,(2 i+1) \delta / 4)$ in the following way (see also Fig. 12):

$$
v_{\varepsilon}(x):=\left\{\begin{aligned}
-\frac{2\left(d-d_{\varepsilon}\right) \delta}{(\delta / 4-\varepsilon T)^{2}}\left|x-(4 i-1) \frac{\delta}{8}+\frac{\varepsilon T}{2}\right| & +\frac{\left(d-d_{\varepsilon}\right) \delta}{(\delta / 4-\varepsilon T)}+1+k \\
& \text { if }(2 i-1) \delta / 4 \leqslant x \leqslant i \delta / 2-\varepsilon T, \\
v_{\varepsilon, 1}^{i}(x) & \text { if } i \delta / 2-\varepsilon T \leqslant x \leqslant(2 i+1) \delta / 4 .
\end{aligned}\right.
$$

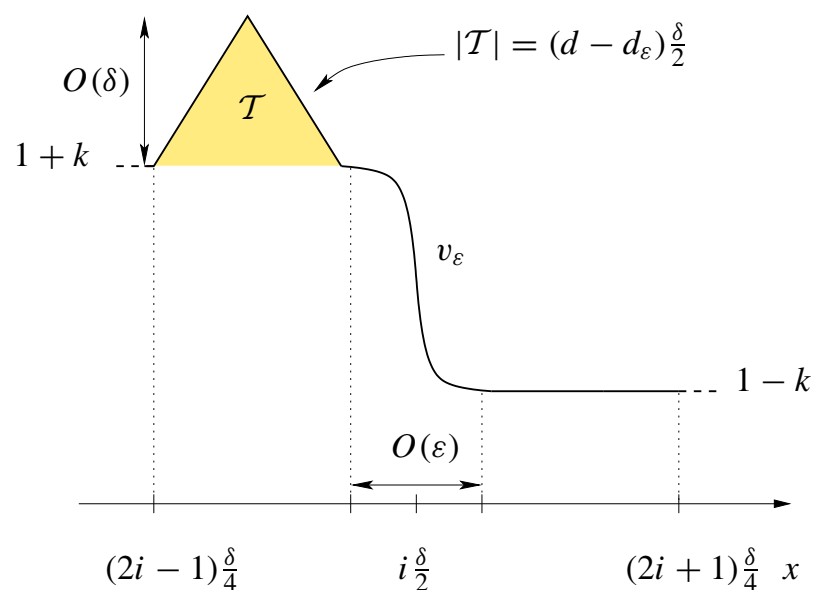

FIG. 12. The recovery sequence $v_{\varepsilon}$ on the interval $((2 i-1) \delta / 4,(2 i+1) \delta / 4)$.

A straightforward computation gives

$$
\begin{aligned}
\frac{\delta}{\varepsilon} \mid \int_{(2 i-1) \delta / 4}^{(2 i+1) \delta / 4} & \left(W^{k}\left(\frac{x}{\varepsilon}, u_{\varepsilon}\right)-W^{k}\left(\frac{x}{\varepsilon}, v_{\varepsilon}\right)\right) \mathrm{d} x \mid \\
& =\frac{\delta}{\varepsilon} \int_{(2 i-1) \delta / 4}^{i \delta / 2-\varepsilon T}\left(-\frac{2\left(d-d_{\varepsilon}\right) \delta}{(\delta / 4-\varepsilon T)^{2}}\left|x-(4 i-1) \frac{\delta}{8}+\frac{\varepsilon T}{2}\right|+\frac{\left(d-d_{\varepsilon}\right) \delta}{(\delta / 4-\varepsilon T)}\right)^{2} \mathrm{~d} x \\
& =\frac{1}{3} \frac{\left(d-d_{\varepsilon}\right)^{2} \delta^{3}}{(\delta / 4-\varepsilon T) \varepsilon}
\end{aligned}
$$

and 


$$
\begin{aligned}
\delta \varepsilon\left|\int_{(2 i-1) \delta / 4}^{(2 i+1) \delta / 4}\left(\left(u_{\varepsilon}^{\prime}\right)^{2}-\left(v_{\varepsilon}^{\prime}\right)^{2}\right) \mathrm{d} x\right| & =\delta \varepsilon \int_{(2 i-1) \delta / 4}^{i \delta / 2-\varepsilon T} \frac{4\left(d-d_{\varepsilon}\right)^{2} \delta^{2}}{(\delta / 4-\varepsilon T)^{4}} \mathrm{~d} x \\
& =\delta \varepsilon \frac{4\left(d-d_{\varepsilon}\right)^{2} \delta^{2}}{(\delta / 4-\varepsilon T)^{4}}(\delta / 4-\varepsilon T) .
\end{aligned}
$$

Since we want a recovery sequence satisfying the volume constraint 4.47, we repeat the above construction (and a similar one for $v_{\varepsilon, 0}^{1}$ ) on each interval of length $\delta / 2$, thus obtaining a sequence $v_{\varepsilon}$ such that

$$
\int_{0}^{1} v_{\varepsilon}(x) \mathrm{d} x=\int_{0}^{1} u_{\varepsilon}(x)+\frac{2}{\delta}\left(d-d_{\varepsilon}\right) \frac{\delta}{2}=d_{\varepsilon}+d-d_{\varepsilon}=d .
$$

Then, in view of 4.48-4.50 we get

$$
F_{\varepsilon}^{k(1)}\left(u_{\varepsilon}\right)-F_{\varepsilon}^{k(1)}\left(v_{\varepsilon}\right)=O\left(\frac{\delta^{3}}{\varepsilon}\right) \quad \text { as } \varepsilon \rightarrow 0 .
$$

which proves the desired convergence under the assumption $\delta \ll \varepsilon^{1 / 3}$.

We now consider the family of integral functionals given by

$$
\mathcal{F}_{\varepsilon}^{k(1)}(u):=F_{\varepsilon}^{k(1)}(u)-\int_{0}^{1} l(u) \mathrm{d} x
$$

where $l$ is a linear function. By the stability of $\Gamma$-convergence under continuous perturbations, we know that 4.51) $\Gamma$-converges to

$$
\mathcal{F}^{k(1)}(u)=F^{k(1)}(u)-\int_{0}^{1} l(u) \mathrm{d} x
$$

for any $u \in L^{2}(0,1)$ such that $|u| \leqslant 1$ a.e. and, under the additional hypothesis $\delta \ll \varepsilon^{1 / 3}$, satisfying the integral constraint $\sqrt{4.47)}$. Since $\mathcal{F}_{\varepsilon}^{k(1)}$ differs from $F_{\varepsilon}^{k(1)}$ by a constant, information on minimizing sequences for $F_{\varepsilon}^{k(1)}$ (satisfying 4.47) ) can be recovered from information on those $\operatorname{minimizing} \mathcal{F}_{\varepsilon}^{k(1)}$.

Notice that in view of the nonstrict convexity of $\psi^{k}$, it is possible to choose the function $l$ in such a way that $\psi^{k}(s)-l(s)$ attains its minimum on a large set. In fact, choosing for instance

$$
l(s)=r^{k}(s):=2\left(C_{1}^{k}-C_{2}^{k}\right) s+2 C_{2}^{k},
$$

by virtue of 4.10 we have

$$
\psi^{k}(s)-r^{k}(s) \geqslant 0 \quad \text { if }|s| \leqslant 1 \quad \text { and } \quad \psi^{k}(s)-r^{k}(s)=0 \quad \text { if } 0<s<1 .
$$

Thus $\min \mathcal{F}^{k(1)}=0=\mathcal{F}^{k(1)}(u)$ for any $u \in L^{2}(0,1)$ with $0 \leqslant u \leqslant 1$ a.e. and $\int_{0}^{1} u \mathrm{~d} x=d$. This means that $\mathcal{F}_{\varepsilon}^{k(1)} \Gamma$-converges to a "degenerate" functional. Hence, we may look for a meaningful scaling for 4.51 and consider

$$
\mathcal{F}_{\varepsilon}^{k(2)}(u):=\frac{\mathcal{F}_{\varepsilon}^{k(1)}(u)}{\bar{\lambda}_{\infty}^{(2)}(\varepsilon)} .
$$


Theorem 4.2. Step 2, combined with the choices $l \equiv r^{k}$ and $d \in(0,1)$, suggests that in this case the relevant transitions are those from $1+k$ to $1-k$ and those from $1-k$ to $-1+k$ (i.e., the transitions with average 1 and 0 , respectively).

Arguing as for $k<1 / 2$, since the passage from oscillations around 1 to oscillations around 0 seems, at a first approximation, energetically negligible, one could guess that (under the usual assumption $\delta \gg e^{-\delta / 2 \varepsilon}$ ) the next meaningful scaling is $\delta$ which now takes into account only the external-boundary-layer effect (see Remark 4.8). A more accurate scale analysis (performed in Theorem 4.10 below) shows that the interaction between these two different types of microscopic phase transitions gives rise to an extra scale that has to be compared with $\delta$. This scale, which turns out to be $\varepsilon / \delta$, takes into account the fact that we are mixing periodic phase transitions with different energy contribution. What happens is that for any fixed $\varepsilon>0$ a minimizer $v_{\varepsilon}$ will be the result of a suitable mixture of oscillations (i.e., periodic transitions) with average $s_{\varepsilon}>0\left(s_{\varepsilon} \rightarrow 0\right.$ as $\left.\varepsilon \rightarrow 0\right)$ and oscillations with average $1+s_{\varepsilon}$. Loosely speaking, using these two averages (instead of 0 and 1), since $v_{\varepsilon}$ has to satisfy the integral constraint (4.47), we can use a smaller proportion of energetically expensive transitions.

Note that if $\delta \gg \varepsilon / \delta$, then the argument in Remark 4.9 for the compatibility of the integral constraint 4.47) with the $\Gamma$-limit of $(1 / \delta) \mathcal{F}_{\varepsilon}^{k(1)}(u)$ cannot be applied. We then turn our attention to the case $\delta \ll \varepsilon / \delta$, for which we have $\bar{\lambda}_{\infty}^{(2)}=\varepsilon / \delta$ and consequently

$$
\mathcal{F}_{\varepsilon}^{k(2)}(u)= \begin{cases}\frac{\delta^{2}}{\varepsilon^{2}} \int_{0}^{1}\left(W^{k}\left(\frac{x}{\delta}, u\right)+\varepsilon^{2}\left(u^{\prime}\right)^{2}\right) \mathrm{d} x-\frac{\delta}{\varepsilon} \int_{0}^{1} r^{k}(u) \mathrm{d} x \\ +\infty & \text { if } u \in W^{1,2}(0,1), \int_{0}^{1} u=d \\ & \text { otherwise }\end{cases}
$$

We prove the following result.

THEOREM 4.10 Let $\delta$ be such that $\delta \ll \varepsilon^{1 / 2}$ and $1 / \delta \in \mathbb{N}$. The family of functionals $\mathcal{F}_{\varepsilon}^{k(2)}$ defined by $4.52 \Gamma$-converges with respect to weak $L^{2}$-convergence to the functional defined on $L^{2}(0,1)$ by

$$
\mathcal{F}^{k(2)}(u)= \begin{cases}-\left(C_{1}^{k}-C_{2}^{k}\right)^{2} & \text { if } u \in L^{2}(0,1), 0 \leqslant u \leqslant 1 \text { a.e., and } \int_{0}^{1} u=d, \\ +\infty & \text { otherwise. }\end{cases}
$$

Before proving the above statement, we need a refinement of the bound on $\varphi_{\eta}^{k}$ in Theorem 4.2 . Step 1, which will be the key ingredient in the proof of Theorem 4.10

LEMMA 4.11 Let $\varphi_{\eta}^{k}$ be defined as in Corollary 4.4 Then

$$
\varphi_{\eta}^{k}(s)= \begin{cases}\frac{s^{2}}{2 \eta}+C_{2}^{k} \tanh \left(\frac{1}{4 \eta}\right) & \text { if }|s| \leqslant c \sqrt{\eta}, \\ \frac{(|s|-1)^{2}}{2 \eta}+C_{1}^{k} \tanh \left(\frac{1}{4 \eta}\right) & \text { if }|s| \geqslant 1-c \sqrt{\eta},\end{cases}
$$

for some positive constant $c$ independent of $\eta$.

Proof. We handle the case of $|s| \leqslant c \sqrt{\eta}$ only (with $c$ suitably chosen), the proof of the other case being analogous. We start by estimating $\varphi_{\eta}^{k}$ from above. By definition, we trivially have 


$$
\begin{aligned}
\varphi_{\eta}^{k}(s) & \leqslant \min \left\{\int_{-1 / 4}^{1 / 4}\left(\frac{1}{\eta} W^{k}(x, u)+\eta\left(u^{\prime}\right)^{2}\right) \mathrm{d} x: u \in W^{1,2}(-1 / 4,1 / 4),\right. \\
\left.f_{-1 / 4}^{1 / 4} u \mathrm{~d} x=s,\|u\|_{\infty} \leqslant k\right\} & \\
& =\min \left\{\int_{-1 / 4}^{1 / 4}\left(\frac{1}{\eta} \mathcal{W}^{k}(x, u)+\eta\left(u^{\prime}\right)^{2}\right) \mathrm{d} x: u \in W^{1,2}(-1 / 4,1 / 4), f_{-1 / 4}^{1 / 4} u \mathrm{~d} x=s\right\}
\end{aligned}
$$

where

$$
\mathcal{W}^{k}(x, u):= \begin{cases}(u-1+k)^{2} & \text { if }-1 / 4 \leqslant x \leqslant 0 \\ (u+1-k)^{2} & \text { if } 0 \leqslant x \leqslant 1 / 4\end{cases}
$$

Following the Lagrange Multipliers Method we explicitly determine the minimum value (4.54) by means of the auxiliary minimum problem

$$
M_{\eta}^{k}(\lambda):=\min \left\{\int_{-1 / 4}^{1 / 4}\left(\frac{1}{\eta} \mathcal{W}^{k}(x, u)+\eta\left(u^{\prime}\right)^{2}+\lambda u\right) \mathrm{d} x: u \in W^{1,2}(-1 / 4,1 / 4)\right\},
$$

with $\lambda \in \mathbb{R}$. Taking into account the definition 4.55 of $\mathcal{W}^{k}$, it is easy to check that $M_{\eta}^{k}(\lambda)$ can be equivalently expressed as

$$
\begin{aligned}
& \min _{u_{0}}\left\{\min \left\{\int_{-1 / 4}^{0}\left(\frac{1}{\eta}(u-1+k)^{2}+\eta\left(u^{\prime}\right)^{2}+\lambda u\right) \mathrm{d} x: u \in W^{1,2}(-1 / 4,0), u(0)=u_{0}\right\}\right. \\
& \left.+\min \left\{\int_{0}^{1 / 4}\left(\frac{1}{\eta}(u+1-k)^{2}+\eta\left(u^{\prime}\right)^{2}+\lambda u\right) \mathrm{d} x: u \in W^{1,2}(0,1 / 4), u(0)=u_{0}\right\}\right\} .
\end{aligned}
$$

Then, by a straightforward computation relying on the associated Euler-Lagrange equations, we find that the minimum (4.56) is attained at

$$
u_{\eta}^{\lambda}(x)= \begin{cases}1-k-\frac{\lambda \eta}{2}+(k-1) \cosh \left(\frac{x}{\eta}\right)+(k-1) \sinh \left(\frac{x}{\eta}\right) \tanh \left(\frac{1}{4 \eta}\right), & -1 / 4 \leqslant x \leqslant 0, \\ -1+k-\frac{\lambda \eta}{2}-(k-1) \cosh \left(\frac{x}{\eta}\right)+(k-1) \sinh \left(\frac{x}{\eta}\right) \tanh \left(\frac{1}{4 \eta}\right), & 0 \leqslant x \leqslant 1 / 4 .\end{cases}
$$

Moreover, in 4.57) the dependence on $\lambda$ can be rewritten in terms of $s$ by imposing the integral constraint

$$
\int_{-1 / 4}^{1 / 4} u_{\eta}^{\lambda}(x) \mathrm{d} x=\frac{s}{2}
$$

which gives $\lambda=-2 s / \eta$. Notice that $u_{\eta}^{-2 s / \eta}=v_{\eta}^{0}+s$, with $v_{\eta}^{0}$ as in 4.12). Finally, evaluating the energy in 4.54 at $u_{\eta}^{-2 s / \eta}$, by a direct computation we get

$$
\varphi_{\eta}^{k}(s) \leqslant \frac{s^{2}}{2 \eta}+C_{2}^{k} \tanh \left(\frac{1}{4 \eta}\right) .
$$

Now we want to prove that there exists a constant $c>0$ such that for $|s| \leqslant c \sqrt{\eta}, \sqrt{4.58}$ ) is an equality. In particular, we show that if $v_{\eta}^{s}$ is a minimizing function for $\varphi_{\eta}^{k}(s)$, then $\left\|v_{\eta}^{s}\right\|_{\infty}<k$. To this end, we additionally assume that $s>0$ (the case $s<0$ being symmetric). 
To start, we claim that supposing $v_{\eta}^{s}(0)=k$ yields a contradiction. In fact, on one hand we have

$$
\begin{aligned}
\varphi_{\eta}^{k}(s) \geqslant & \min \left\{\int_{-1 / 4}^{0}\left(\frac{1}{\eta}(u-1+k)^{2}+\eta\left(u^{\prime}\right)^{2}\right) \mathrm{d} x: u \in W^{1,2}(-1 / 4,0), u(0)=k\right\} \\
& +\min \left\{\int_{0}^{1 / 4}\left(\frac{1}{\eta}(u+1-k)^{2}+\eta\left(u^{\prime}\right)^{2}\right) \mathrm{d} x: u \in W^{1,2}(0,1 / 4), u(0)=k\right\} \\
= & \tanh \left(\frac{1}{4 \eta}\right)+(2 k-1)^{2} \tanh \left(\frac{1}{4 \eta}\right) \\
= & 1+(2 k-1)^{2}+\left(1+(2 k-1)^{2}\right)\left(\tanh \left(\frac{1}{4 \eta}\right)-1\right) \\
= & C_{1}^{k}+C_{2}^{k}+o(1) \quad \text { as } \eta \rightarrow 0 .
\end{aligned}
$$

On the other hand, from 4.58, if $0<s \leqslant c \sqrt{\eta}$, we also find

$$
\varphi_{\eta}^{k}(s) \leqslant c / 2+C_{2}^{k}+o(1) .
$$

As a consequence if we choose $c<2 C_{1}^{k}$, combining 4.59 and 4.60 we get a contradiction, and the claim follows.

It is easy to check that the case $v_{\eta}^{s}(0)=k$ is the most "convenient" one (in terms of the minimum problem defining $\varphi_{\eta}^{k}$ ) among those for which the function $v_{\eta}^{s}$ does not satisfy $\left\|v_{\eta}^{s}\right\|<k$. So in particular this excludes the existence of a point $x_{\eta} \in(-1 / 4,1 / 4), x_{\eta} \neq 0$, such that $v_{\eta}^{s}\left(x_{\eta}\right) \geqslant k$. Moreover, the additional hypothesis $s>0$ combined with the previous argument also excludes $v_{\eta}^{s}\left(x_{\eta}\right) \leqslant-k$ for some $x_{\eta} \in(-1 / 4,1 / 4)$, which would clearly be even more unfavorable. This concludes the proof of the lemma for $s>0$.

Proof of Theorem 4.10. Step 1: $\Gamma$-liminf inequality. We prove that if $u_{\varepsilon} \rightarrow u$ in $L^{2}(0,1)$ and $\sup _{\varepsilon} \mathcal{F}_{\varepsilon}^{k(2)}\left(u_{\varepsilon}\right)<+\infty$, then $\mathcal{F}^{k(2)}(u) \leqslant \liminf _{\varepsilon \rightarrow 0} \mathcal{F}_{\varepsilon}^{k(2)}\left(u_{\varepsilon}\right)$. Notice that, in view of the definition of $\mathcal{F}_{\varepsilon}^{k(2)}$, we immediately have $0 \leqslant u \leqslant 1$ a.e.

We start by writing $\mathcal{F}_{\varepsilon}^{k(2)}$ as the sum of three terms,

$$
\begin{aligned}
\mathcal{F}_{\varepsilon}^{k(2)}\left(u_{\varepsilon}\right)= & \frac{\delta^{2}}{\varepsilon^{2}} \int_{0}^{\delta / 4}\left(W_{1}^{k}\left(u_{\varepsilon}\right)+\varepsilon^{2}\left(u_{\varepsilon}^{\prime}\right)^{2}\right) \mathrm{d} x-\frac{\delta}{\varepsilon} \int_{0}^{\delta / 4} r^{k}\left(u_{\varepsilon}\right) \mathrm{d} x \\
& +\sum_{i=1}^{2 / \delta-1}\left(\frac{\delta^{2}}{\varepsilon^{2}} \int_{(2 i-1) \delta / 4}^{(2 i+1) \delta / 4}\left(W^{k}\left(\frac{x}{\delta}, u_{\varepsilon}\right)+\varepsilon^{2}\left(u_{\varepsilon}^{\prime}\right)^{2}\right) \mathrm{d} x-\frac{\delta}{\varepsilon} \int_{(2 i-1) \delta / 4}^{(2 i+1) \delta / 4} r^{k}\left(u_{\varepsilon}\right) \mathrm{d} x\right) \\
& +\frac{\delta^{2}}{\varepsilon^{2}} \int_{1-\delta / 4}^{1}\left(W_{2}^{k}\left(u_{\varepsilon}\right)+\varepsilon^{2}\left(u_{\varepsilon}^{\prime}\right)^{2}\right) \mathrm{d} x-\frac{\delta}{\varepsilon} \int_{1-\delta / 4}^{1} r^{k}\left(u_{\varepsilon}\right) \mathrm{d} x,
\end{aligned}
$$

and we set

$$
\begin{aligned}
& I_{\varepsilon}^{1}:=\frac{\delta^{2}}{\varepsilon^{2}} \int_{0}^{\delta / 4}\left(W_{1}^{k}\left(u_{\varepsilon}\right)+\varepsilon^{2}\left(u_{\varepsilon}^{\prime}\right)^{2}\right) \mathrm{d} x-\frac{\delta}{\varepsilon} \int_{0}^{\delta / 4} r^{k}\left(u_{\varepsilon}\right) \mathrm{d} x, \\
& I_{\varepsilon}^{2}:=\frac{\delta^{2}}{\varepsilon^{2}} \int_{1-\delta / 4}^{1}\left(W_{2}^{k}\left(u_{\varepsilon}\right)+\varepsilon^{2}\left(u_{\varepsilon}^{\prime}\right)^{2}\right) \mathrm{d} x-\frac{\delta}{\varepsilon} \int_{1-\delta / 4}^{1} r^{k}\left(u_{\varepsilon}\right) \mathrm{d} x .
\end{aligned}
$$


Hence

$$
\begin{aligned}
& \liminf _{\varepsilon \rightarrow 0} \mathcal{F}_{\varepsilon}^{k(2)}\left(u_{\varepsilon}\right) \geqslant \liminf _{\varepsilon \rightarrow 0} I_{\varepsilon}^{1}+\liminf _{\varepsilon \rightarrow 0} I_{\varepsilon}^{2} \\
& \quad+\liminf _{\varepsilon \rightarrow 0}^{2 / \delta-1} \sum_{i=1}^{(2 i+1) \delta / 4}\left(\frac{\delta^{2}}{\varepsilon^{2}} \int_{(2 i-1) \delta / 4}^{(2 i)}\left(W^{k}\left(\frac{x}{\delta}, u_{\varepsilon}\right)+\varepsilon^{2}\left(u_{\varepsilon}^{\prime}\right)^{2}\right) \mathrm{d} x-\frac{\delta}{\varepsilon} \int_{(2 i-1) \delta / 4}^{(2 i+1) \delta / 4} r^{k}\left(u_{\varepsilon}\right) \mathrm{d} x\right) .
\end{aligned}
$$

We now claim that

$$
\liminf _{\varepsilon \rightarrow 0} I_{\varepsilon}^{1} \geqslant 0 \quad \text { and } \quad \liminf _{\varepsilon \rightarrow 0} I_{\varepsilon}^{2} \geqslant 0 .
$$

We prove this claim only for $I_{\varepsilon}^{1}$, the proof for $I_{\varepsilon}^{2}$ being analogous. Let $\bar{u}_{\varepsilon}:=f_{0}^{\delta / 4} u_{\varepsilon} \mathrm{d} x$. Then, recalling that $\delta \gg \varepsilon$,

$$
\begin{aligned}
\liminf _{\varepsilon \rightarrow 0} I_{\varepsilon}^{1} & \geqslant \liminf _{\varepsilon \rightarrow 0} \frac{\delta^{2}}{4 \varepsilon}\left(\frac{\delta}{\varepsilon}\left(W_{1}^{k}\right)^{* *}\left(\bar{u}_{\varepsilon}\right)-r^{k}\left(\bar{u}_{\varepsilon}\right)\right) \geqslant \liminf _{\varepsilon \rightarrow 0} \frac{\delta^{2}}{4 \varepsilon}\left(\left(W_{1}^{k}\right)^{* *}\left(\bar{u}_{\varepsilon}\right)-r^{k}\left(\bar{u}_{\varepsilon}\right)\right) \\
& \geqslant \liminf _{\varepsilon \rightarrow 0} \frac{\delta^{2}}{4 \varepsilon} \min _{s \in \mathbb{R}}\left(\left(W_{1}^{k}\right)^{* *}(s)-r^{k}(s)\right)=\liminf _{\varepsilon \rightarrow 0} \frac{\delta^{2}}{\varepsilon}\left(-7 k^{2}+5 k-1\right)=0
\end{aligned}
$$

where the last equality follows by hypothesis. Thus we get

$$
\begin{aligned}
& \liminf _{\varepsilon \rightarrow 0} \mathcal{F}_{\varepsilon}^{k(2)}\left(u_{\varepsilon}\right) \\
& \geqslant \liminf _{\varepsilon \rightarrow 0} \sum_{i=1}^{2 / \delta-1}\left(\frac{\delta^{2}}{\varepsilon^{2}} \int_{(2 i-1) \delta / 4}^{(2 i+1) \delta / 4}\left(W^{k}\left(\frac{x}{\delta}, u_{\varepsilon}\right)+\varepsilon^{2}\left(u_{\varepsilon}^{\prime}\right)^{2}\right) \mathrm{d} x-\frac{\delta}{\varepsilon} \int_{(2 i-1) \delta / 4}^{(2 i+1) \delta / 4} r^{k}\left(u_{\varepsilon}\right) \mathrm{d} x\right) \\
& \geqslant \liminf _{\varepsilon \rightarrow 0} \frac{\delta}{\varepsilon} \sum_{i=1}^{2 / \delta-1} \frac{\delta}{2}\left(\int_{(2 i-1) \delta / 4}^{(2 i+1) \delta / 4} 2\left(\frac{1}{\varepsilon} W^{k}\left(\frac{x}{\delta}, u_{\varepsilon}\right)+\varepsilon\left(u_{\varepsilon}^{\prime}\right)^{2}\right) \mathrm{d} x-f_{(2 i-1) \delta / 4}^{(2 i+1) \delta / 4} r^{k}\left(u_{\varepsilon}\right) \mathrm{d} x\right) \\
& \geqslant \liminf _{\varepsilon \rightarrow 0} \frac{\delta}{\varepsilon} \sum_{i=1}^{2 / \delta-1} \frac{\delta}{2}\left(2 \varphi_{\varepsilon / \delta}^{k}\left(\tilde{u}_{\varepsilon}\right)-r^{k}\left(\tilde{u}_{\varepsilon}\right)\right),
\end{aligned}
$$

with $\varphi_{\varepsilon / \delta}^{k}$ as in Step 1 of Theorem 4.2 and $\tilde{u}_{\varepsilon}:(0,1) \rightarrow \mathbb{R}$ defined by

$$
\tilde{u}_{\varepsilon}(x):=\sum_{i=1}^{2 / \delta-1}\left(f_{(2 i-1) \delta / 4}^{(2 i+1) \delta / 4} u_{\varepsilon} \mathrm{d} t\right) \chi_{((2 i-1) \delta / 4,(2 i+1) \delta / 4)}(x) .
$$

Notice that by virtue of Lemma 4.11 .

$$
2 \varphi_{\varepsilon / \delta}^{k}(0)-r^{k}(0)=O\left(e^{-\delta / 2 \varepsilon}\right) \quad \text { as } \varepsilon \rightarrow 0 .
$$

Then in view of the definition of $\tilde{u}_{\varepsilon}$ we deduce that

$$
\lim _{\varepsilon \rightarrow 0} \frac{\delta}{\varepsilon} \int_{0}^{\delta / 4}\left(2 \varphi_{\varepsilon / \delta}^{k}\left(\tilde{u}_{\varepsilon}\right)-r^{k}\left(\tilde{u}_{\varepsilon}\right)\right) \mathrm{d} x=\lim _{\varepsilon \rightarrow 0} \frac{\delta}{\varepsilon} \int_{1-\delta / 4}^{1}\left(2 \varphi_{\varepsilon / \delta}^{k}\left(\tilde{u}_{\varepsilon}\right)-r^{k}\left(\tilde{u}_{\varepsilon}\right)\right) \mathrm{d} x=0
$$


and consequently

$$
\liminf _{\varepsilon \rightarrow 0} \mathcal{F}_{\varepsilon}^{k(2)}\left(u_{\varepsilon}\right) \geqslant \liminf _{\varepsilon \rightarrow 0} \frac{\delta}{\varepsilon} \int_{0}^{1}\left(2 \varphi_{\varepsilon / \delta}^{k}\left(\tilde{u}_{\varepsilon}\right)-r^{k}\left(\tilde{u}_{\varepsilon}\right)\right) \mathrm{d} x .
$$

So now we need to estimate the function $2 \varphi_{\varepsilon / \delta}^{k}(s)-r^{k}(s)$ from below. Since the estimate on $\varphi_{\varepsilon / \delta}^{k}$ already established in Theorem 4.2. Step 1 is too coarse to be used at this scale, we need to refine it. By means of Lemma 4.11, we start by improving this estimate in a neighborhood of $s=0$. To this end, for (small) fixed $\sigma>0$ we consider those $s$ such that $|s|<\sigma$ and we denote by $v_{\varepsilon}^{s}$ a minimizing function for $\varphi_{\varepsilon / \delta}^{k}(s)$. Arguing as in Lemma 4.11 for $\left\|v_{\varepsilon}^{s}\right\|_{\infty}<k$ we have

$$
\varphi_{\varepsilon / \delta}^{k}(s)=\frac{\delta}{2 \varepsilon} s^{2}+C_{2}^{k} \tanh \left(\frac{\delta}{4 \varepsilon}\right),
$$

while for $\left\|v_{\varepsilon}^{s}\right\|_{\infty} \geqslant k$ it is easily seen that the combined argument of Theorem 4.2, Step 1 and Lemma 4.11 yields

$$
\varphi_{\varepsilon / \delta}^{k}(s) \geqslant C_{1}^{k}+C_{2}^{k}-C \sigma^{2} .
$$

Thus, for every $s$ such that $|s|<\sigma$ we have

$$
\begin{aligned}
\varphi_{\varepsilon / \delta}^{k}(s) & \geqslant \min \left\{\frac{\delta}{2 \varepsilon} s^{2}+C_{2}^{k} \tanh \left(\frac{\delta}{4 \varepsilon}\right), C_{1}^{k}+C_{2}^{k}-C \sigma^{2}\right\} \\
& = \begin{cases}\frac{\delta}{2 \varepsilon} s^{2}+C_{2}^{k} \tanh \left(\frac{\delta}{4 \varepsilon}\right) & \text { if }|s|<s_{\varepsilon, \sigma}^{0}, \\
C_{1}^{k}+C_{2}^{k}-C \sigma^{2} & \text { if } s_{\varepsilon, \sigma}^{0}<|s|<\sigma,\end{cases}
\end{aligned}
$$

with

$$
s_{\varepsilon, \sigma}^{0}:=\sqrt{\frac{\varepsilon}{\delta}}\left(2 C_{2}^{k}\left(1-\tanh \left(\frac{\delta}{4 \varepsilon}\right)\right)+2 C_{1}^{k}-2 C \sigma^{2}\right)^{1 / 2}=O\left(\sqrt{\frac{\varepsilon}{\delta}}\right) \quad \text { as } \varepsilon \rightarrow 0 .
$$

A similar analysis can be performed for $\sigma<|s| \leqslant 1$, giving

$$
\begin{aligned}
\varphi_{\varepsilon / \delta}^{k}(s) & \geqslant \min \left\{\frac{\delta}{2 \varepsilon}(|s|-1)^{2}+C_{1}^{k} \tanh \left(\frac{\delta}{4 \varepsilon}\right), C_{1}^{k}+C_{2}^{k}-C \sigma^{2}\right\} \\
& = \begin{cases}\frac{\delta}{2 \varepsilon}(|s|-1)^{2}+C_{1}^{k} \tanh \left(\frac{\delta}{4 \varepsilon}\right) & \text { if } s_{\varepsilon, \sigma}^{1} \leqslant|s| \leqslant 1, \\
C_{1}^{k}+C_{2}^{k}-C \sigma^{2} & \text { if } \sigma \leqslant s<s_{\varepsilon, \sigma}^{1},\end{cases}
\end{aligned}
$$

with

$$
s_{\varepsilon, \sigma}^{1}:=1-\sqrt{\frac{\varepsilon}{\delta}}\left(2 C_{1}^{k}\left(1-\tanh \left(\frac{\delta}{4 \varepsilon}\right)\right)+2 C_{1}^{k}-2 C \sigma^{2}\right)^{1 / 2} .
$$

Hence, combinng 4.61, 4.62) and Lemma 4.11 (for $|s|>1$ ), for every $s \in \mathbb{R}$ we derive the following estimate:

$$
\varphi_{\varepsilon / \delta}^{k}(s) \geqslant \phi_{\delta / \varepsilon, \sigma}^{k}(s):= \begin{cases}\frac{\delta}{2 \varepsilon} s^{2}+C_{2}^{k} \tanh \left(\frac{\delta}{4 \varepsilon}\right) & \text { if }|s|<s_{\varepsilon, \sigma}^{0}, \\ C_{1}^{k}+C_{2}^{k}-C \sigma^{2} & \text { if } s_{\varepsilon, \sigma}^{0}<|s|<s_{\varepsilon, \sigma}^{1}, \\ \frac{\delta}{2 \varepsilon}(|s|-1)^{2}+C_{1}^{k} \tanh \left(\frac{\delta}{4 \varepsilon}\right) & \text { if }|s|>s_{\varepsilon, \sigma}^{1} .\end{cases}
$$




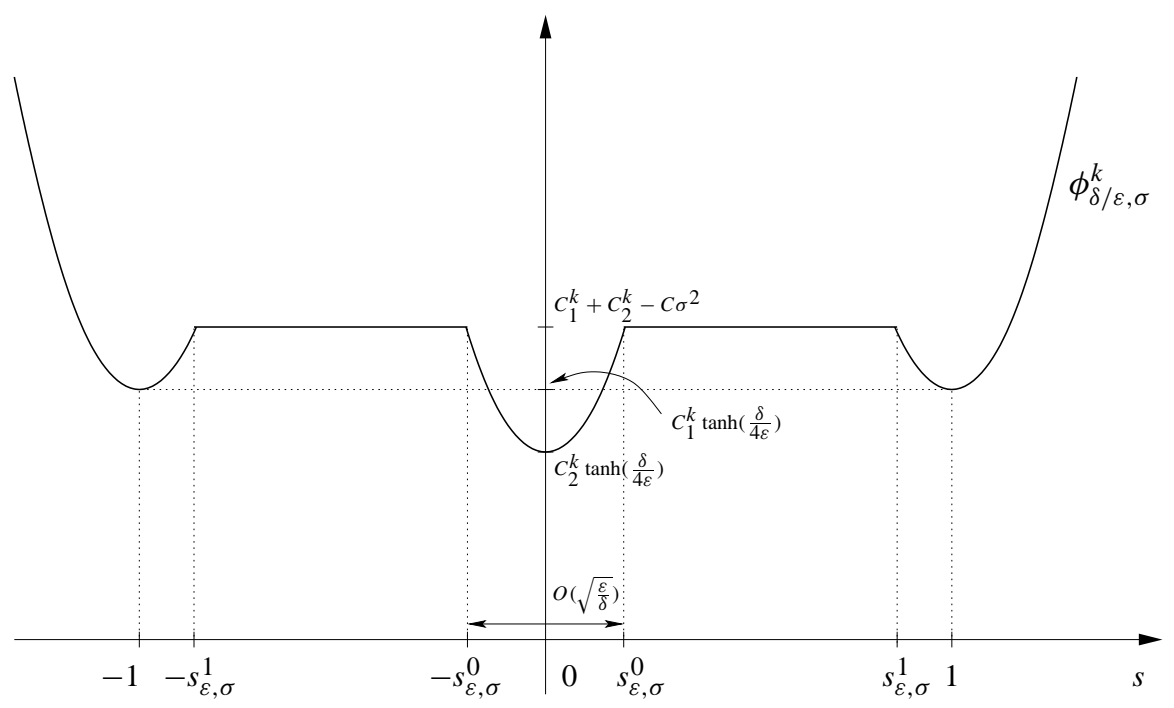

FIG. 13. The function $\phi_{\delta / \varepsilon, \sigma}^{k}$.

As a consequence we get

$$
\begin{aligned}
\liminf _{\varepsilon \rightarrow 0} \mathcal{F}_{\varepsilon}^{k(2)}\left(u_{\varepsilon}\right) & \geqslant \liminf _{\varepsilon \rightarrow 0} \frac{\delta}{\varepsilon} \int_{0}^{1}\left(2 \phi_{\delta / \varepsilon, \sigma}^{k}\left(\tilde{u}_{\varepsilon}\right)-r^{k}\left(\tilde{u}_{\varepsilon}\right)\right) \mathrm{d} x \\
& \geqslant \liminf _{\varepsilon \rightarrow 0} \frac{\delta}{\varepsilon} \int_{0}^{1}\left(2\left(\phi_{\delta / \varepsilon, \sigma}^{k}\right)^{* *}\left(\tilde{u}_{\varepsilon}\right)-r^{k}\left(\tilde{u}_{\varepsilon}\right)\right) \mathrm{d} x
\end{aligned}
$$

where

$$
\left(\phi_{\delta / \varepsilon, \sigma}^{k}\right)^{* *}(s)= \begin{cases}\frac{\delta}{2 \varepsilon} s^{2}+C_{2}^{k} \tanh \left(\frac{\delta}{4 \varepsilon}\right) & \text { if }|s| \leqslant \bar{s}_{\varepsilon}, \\ \left(C_{1}^{k}-C_{2}^{k}\right) \tanh \left(\frac{\delta}{4 \varepsilon}\right)|s|+C_{2}^{k} \tanh \left(\frac{\delta}{4 \varepsilon}\right)-\frac{\varepsilon}{2 \delta}\left(C_{1}^{k}-C_{2}^{k}\right)^{2} \tanh ^{2}\left(\frac{\delta}{4 \varepsilon}\right) \\ \frac{\delta}{2 \varepsilon}(|s|-1)^{2}+C_{1}^{k} \tanh \left(\frac{\delta}{4 \varepsilon}\right) & \text { if } \bar{s}_{\varepsilon}<|s|<1+\bar{s}_{\varepsilon},\end{cases}
$$

with $\bar{s}_{\varepsilon}:=(\varepsilon / \delta)\left(C_{1}^{k}-C_{2}^{k}\right) \tanh (\delta / 4 \varepsilon)$. Since the sequence $\left((\delta / \varepsilon)\left(2\left(\phi_{\delta / \varepsilon, \sigma}^{k}\right)^{* *}(s)-r^{k}(s)\right)\right)$ increases with $\delta / \varepsilon$, for any fixed $m>0$ there exists $\varepsilon_{0}>0$ such that

$$
\frac{\delta}{\varepsilon}\left(2\left(\phi_{\delta / \varepsilon, \sigma}^{k}\right)^{* *}(s)-r^{k}(s)\right)>m\left(2\left(\phi_{m, \sigma}^{k}\right)^{* *}(s)-r^{k}(s)\right) \quad \text { for all } \varepsilon<\varepsilon_{0} .
$$

Then by lower semicontinuity,

$\liminf _{\varepsilon \rightarrow 0} \mathcal{F}_{\varepsilon}^{k(2)}\left(u_{\varepsilon}\right) \geqslant \liminf _{\varepsilon \rightarrow 0} m \int_{0}^{1}\left(2\left(\phi_{m, \sigma}^{k}\right)^{* *}\left(\tilde{u}_{\varepsilon}\right)-r^{k}\left(\tilde{u}_{\varepsilon}\right)\right) \mathrm{d} x \geqslant m \int_{0}^{1}\left(2\left(\phi_{m, \sigma}^{k}\right)^{* *}(u)-r^{k}(u)\right) \mathrm{d} x$. 
Finally, since it can be easily checked that

$$
\lim _{m \rightarrow+\infty} m\left(2\left(\phi_{m, \sigma}^{k}\right)^{* *}(s)-r^{k}(s)\right)=f(s):= \begin{cases}0 & \text { if } s=0,1, \\ -\left(C_{1}^{k}-C_{2}^{k}\right)^{2} & \text { if } 0<s<1,\end{cases}
$$

a direct application of the Monotone Convergence Theorem gives

$$
\liminf _{\varepsilon \rightarrow 0} \mathcal{F}_{\varepsilon}^{k(2)}\left(u_{\varepsilon}\right) \geqslant \int_{0}^{1} f(u) \mathrm{d} x
$$

thus we have

$$
\liminf _{\varepsilon \rightarrow 0} \mathcal{F}_{\varepsilon}^{k(2)}\left(u_{\varepsilon}\right) \geqslant-\left(C_{1}^{k}-C_{2}^{k}\right)^{2},
$$

proving the $\Gamma$-liminf inequality.

In view of the analysis performed above, to better explain the presence of the scaling $\bar{\lambda}_{\infty}^{(2)}(\varepsilon)=\varepsilon / \delta$, we remark that the final effect of subtracting $r^{k}$ from the original potential $W^{k}$ is that of considering, in place of

$$
\frac{\delta}{\varepsilon} s^{2}+2 C_{2}^{k} \tanh \left(\frac{\delta}{4 \varepsilon}\right), \quad \frac{\delta}{\varepsilon}(s-1)^{2}+2 C_{1}^{k} \tanh \left(\frac{\delta}{4 \varepsilon}\right),
$$

the two parabolas

$$
\begin{gathered}
\frac{\delta}{\varepsilon} s^{2}-2\left(C_{1}^{k}-C_{2}^{k}\right) s+2 C_{2}^{k}\left(\tanh \left(\frac{\delta}{4 \varepsilon}\right)-1\right), \\
\frac{\delta}{\varepsilon}(s-1)^{2}-2\left(C_{1}^{k}-C_{2}^{k}\right)(s-1)+2 C_{1}^{k}\left(\tanh \left(\frac{\delta}{4 \varepsilon}\right)-1\right)
\end{gathered}
$$

with vertices respectively at

$$
\begin{aligned}
V_{0} & \equiv\left(\frac{\varepsilon}{\delta}\left(C_{1}^{k}-C_{2}^{k}\right) ;-\frac{\varepsilon}{\delta}\left(C_{1}^{k}-C_{2}^{k}\right)^{2}+2 C_{2}^{k}\left(\tanh \left(\frac{\delta}{4 \varepsilon}\right)-1\right)\right), \\
V_{1} & \equiv\left(\frac{\varepsilon}{\delta}\left(C_{1}^{k}-C_{2}^{k}\right)+1 ;-\frac{\varepsilon}{\delta}\left(C_{1}^{k}-C_{2}^{k}\right)^{2}+2 C_{1}^{k}\left(\tanh \left(\frac{\delta}{4 \varepsilon}\right)-1\right)\right) .
\end{aligned}
$$

Then, for instance, from

$$
-\frac{\varepsilon}{\delta}\left(C_{1}^{k}-C_{2}^{k}\right)^{2}+2 C_{2}^{k}\left(\tanh \left(\frac{\delta}{4 \varepsilon}\right)-1\right)=O\left(\frac{\varepsilon}{\delta}\right)+O\left(e^{-\delta / 2 \varepsilon}\right)=O\left(\frac{\varepsilon}{\delta}\right) \quad \text { as } \varepsilon \rightarrow 0
$$

we deduce that the correction due to the translation by $r^{k}$ is actually visible at scale $\varepsilon / \delta$.

Step 2: $\Gamma$-limsup inequality. To prove the limsup inequality, it is enough to deal with constant target functions, since the case of piecewise constants can be treated similarly; then the general case follows by density. Since the (constant) target function has to satisfy the volume constraint, we only deal with the case $u \equiv d$.

Let $v_{\varepsilon}^{0}, v_{\varepsilon}^{1}$ be respectively as in 4.12, 4.13 with $\eta=\varepsilon / \delta$, and set $v_{\varepsilon}^{s_{\varepsilon}}:=v_{\varepsilon}^{0}+s_{\varepsilon}, v_{\varepsilon}^{1+s_{\varepsilon}}:=$ $v_{\varepsilon}^{1}+s_{\varepsilon}$, with $s_{\varepsilon}=(\varepsilon / \delta)\left(C_{1}^{k}-C_{2}^{k}\right)$. Then it is easy to check that $v_{\varepsilon}^{s_{\varepsilon}}$ and $v_{\varepsilon}^{1+s_{\varepsilon}}$ are minimizing 
functions for $\varphi_{\varepsilon / \delta}^{k}\left(s_{\varepsilon}\right)$ and $\varphi_{\varepsilon / \delta}^{k}\left(1+s_{\varepsilon}\right)$, respectively (see also the proof of Lemma 4.11 , while in view of 4.63 we get

$$
\begin{aligned}
2 \varphi_{\varepsilon / \delta}^{k}\left(s_{\varepsilon}\right)-r^{k}\left(s_{\varepsilon}\right) & =-\frac{\varepsilon}{\delta}\left(C_{1}^{k}-C_{2}^{k}\right)^{2}+2 C_{2}^{k}\left(\tanh \left(\frac{\delta}{4 \varepsilon}\right)-1\right), \\
2 \varphi_{\varepsilon / \delta}^{k}\left(1+s_{\varepsilon}\right)-r^{k}\left(1+s_{\varepsilon}\right) & =-\frac{\varepsilon}{\delta}\left(C_{1}^{k}-C_{2}^{k}\right)^{2}+2 C_{1}^{k}\left(\tanh \left(\frac{\delta}{4 \varepsilon}\right)-1\right) .
\end{aligned}
$$

Now, arguing as in the proof of Theorem 4.2. Step 2, we consider two sequences of positive integers $\left(n_{1}^{\nu}\right),\left(n_{2}^{\nu}\right)$ such that

$$
n_{1}^{v}, n_{2}^{v} \rightarrow+\infty \quad \text { and } \quad \frac{n_{1}^{v}}{n_{2}^{v}} \rightarrow \frac{d}{1-d} \quad \text { as } v \rightarrow 0 .
$$

For fixed $v>0$, for any sufficiently small $\varepsilon>0$ we have $\left(n_{1}^{v}+n_{2}^{v}+2\right) \delta \ll 1$. We consider the $\left(n_{1}^{v}+n_{2}^{v}+2\right) \delta$-periodic function $u_{\varepsilon}^{v}$, on $\mathbb{R}^{+}$, which on $\left(\delta / 4,\left(4\left(n_{1}^{v}+n_{2}^{v}+1\right)+5\right) \delta / 4\right)$ is defined as

$u_{\varepsilon}^{v}(x):= \begin{cases}u_{\varepsilon}^{1+s_{\varepsilon}}(x), & x \in\left(\delta / 4,\left(4 n_{1}^{v}+1\right) \delta / 4\right), \\ z_{\varepsilon}(x), & x \in\left(\left(4 n_{1}^{v}+1\right) \delta / 4,\left(4 n_{1}^{v}+5\right) \delta / 4\right), \\ u_{\varepsilon}^{s_{\varepsilon}}(x), & x \in\left(\left(4 n_{1}^{v}+5\right) \delta / 4,\left(4\left(n_{1}^{v}+n_{2}^{v}\right)+5\right) \delta / 4\right), \\ z_{\varepsilon}\left(\left(4 n_{1}^{v}+2 n_{2}^{v}+5\right) \delta / 2-x\right), & \left.\left.x \in\left(\left(4\left(n_{1}^{v}+n_{2}^{v}\right)+5\right) \delta / 4\right),\left(4\left(n_{1}^{v}+n_{2}^{v}+1\right)+5\right) \delta / 4\right)\right),\end{cases}$

where

$$
u_{\varepsilon}^{1+s_{\varepsilon}}(x):=\left\{\begin{array}{lll}
v_{\varepsilon}^{1+s_{\varepsilon}}\left(i-\frac{1}{2}-\frac{x}{\delta}\right), & x \in((4 i-3) \delta / 4,(4 i-1) \delta / 4), & \\
v_{\varepsilon}^{1+s_{\varepsilon}}\left(\frac{x}{\delta}-i\right), & x \in((4 i-1) \delta / 4,(4 i+1) \delta / 4), &
\end{array} \quad i=1, \ldots, n_{1}^{\nu},\right.
$$

and

$$
u_{\varepsilon}^{s_{\varepsilon}}(x):=\left\{\begin{array}{ll}
v_{\varepsilon}^{s_{\varepsilon}}\left(i-\frac{1}{2}-\frac{x}{\delta}\right), & x \in\left((4 i-3) \frac{\delta}{4},(4 i-1) \delta / 4\right), \\
v_{\varepsilon}^{s_{\varepsilon}}\left(\frac{x}{\delta}-i\right), & x \in((4 i-1) \delta / 4,(4 i+1) \delta / 4),
\end{array} \quad i=n_{1}^{v}+1, \ldots, n_{1}^{v}+n_{2}^{v}+1 .\right.
$$

The joining transition $z_{\varepsilon}$ is defined as follows:

$$
z_{\varepsilon}(x):= \begin{cases}v_{\varepsilon}^{1+s_{\varepsilon}}\left(n_{1}^{\nu}+\frac{1}{2}-\frac{x}{\delta}\right), & x \in\left(\left(4 n_{1}^{\nu}+1\right) \delta / 4, x_{\varepsilon}^{\prime}\right), \\ \frac{x}{\delta}+q_{\varepsilon}, & x \in\left(x_{\varepsilon}^{\prime}, x_{\varepsilon}^{\prime \prime}\right) \\ v_{\varepsilon}^{s_{\varepsilon}}\left(\frac{x}{\delta}-n_{1}^{\nu}-1\right), & x \in\left(x_{\varepsilon}^{\prime \prime},\left(4 n_{1}^{\nu}+5\right) \delta / 4\right),\end{cases}
$$

with $q_{\varepsilon}$ (and consequently $x_{\varepsilon}^{\prime}, x_{\varepsilon}^{\prime \prime}$ ) chosen in such a way that

$$
\int_{\left(4 n_{1}^{v}+1\right) \delta / 4}^{\left(4 n_{1}^{v}+5\right) \delta / 4} z_{\varepsilon}(x) \mathrm{d} x=\int_{\left(4 n_{1}^{v}+1\right) \delta / 4}^{\left(4 n_{1}^{v}+3\right) \delta / 4} v_{\varepsilon}^{1+s_{\varepsilon}}\left(n_{1}^{v}+\frac{1}{2}-\frac{x}{\delta}\right) \mathrm{d} x+\int_{\left(4 n_{1}^{v}+3\right) \delta / 4}^{\left(4 n_{1}^{v}+5\right) \delta / 4} v_{\varepsilon}^{s_{\varepsilon}}\left(\frac{x}{\delta}-n_{1}^{v}-1\right) \mathrm{d} x .
$$




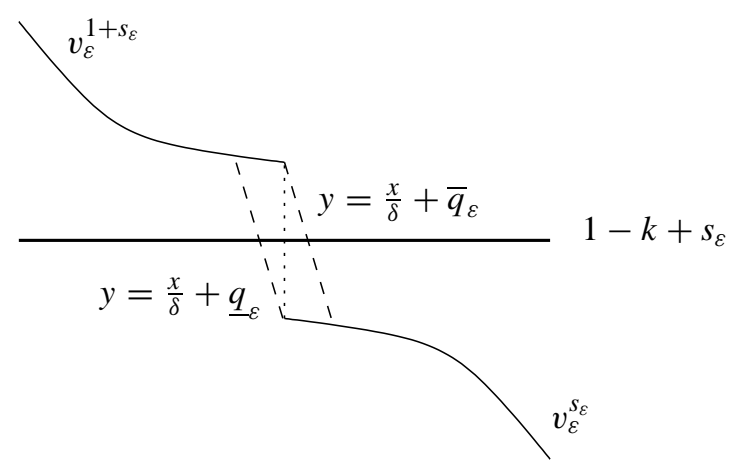

FIG. 14. The mismatch between $v_{\varepsilon}^{1+s_{\varepsilon}}$ and $v_{\varepsilon}^{s_{\varepsilon}}$.

In fact, if we set

$$
I\left(q_{\varepsilon}\right):=\int_{\left(4 n_{1}^{v}+1\right) \delta / 4}^{\left(4 n_{1}^{v}+5\right) \delta / 4} z_{\varepsilon}(x) \mathrm{d} x,
$$

it can be checked (see also Fig. 14 that for $\bar{q}_{\varepsilon}:=1-k+s_{\varepsilon}+(k-1)(\cosh (\delta / 4 \varepsilon))^{-1}-\left(4 n_{1}^{v}+3\right) / 4$,

$$
I\left(\bar{q}_{\varepsilon}\right) \geqslant \int_{\left(4 n_{1}^{v}+1\right) \delta / 4}^{\left(4 n_{1}^{v}+3\right) \delta / 4} v_{\varepsilon}^{1+s_{\varepsilon}}\left(n_{1}^{v}+\frac{1}{2}-\frac{x}{\delta}\right) \mathrm{d} x+\int_{\left(4 n_{1}^{v}+3\right) \delta / 4}^{\left(4 n_{1}^{v}+5\right) \delta / 4} v_{\varepsilon}^{s_{\varepsilon}}\left(\frac{x}{\delta}-n_{1}^{v}-1\right) \mathrm{d} x,
$$

while for $\underline{q}_{\varepsilon}:=1-k+s_{\varepsilon}-(k-1)(\cosh (\delta / 4 \varepsilon))^{-1}-\left(4 n_{1}^{v}+3\right) / 4$ we have

$$
I\left(\underline{q}_{\varepsilon}\right) \leqslant \int_{\left(4 n_{1}^{v}+1\right) \delta / 4}^{\left(4 n_{1}^{v}+3\right) \delta / 4} v_{\varepsilon}^{1+s_{\varepsilon}}\left(n_{1}^{v}+\frac{1}{2}-\frac{x}{\delta}\right) \mathrm{d} x+\int_{\left(4 n_{1}^{v}+3\right) \delta / 4}^{\left(4 n_{1}^{v}+5\right) \delta / 4} v_{\varepsilon}^{s_{\varepsilon}}\left(\frac{x}{\delta}-n_{1}^{v}-1\right) \mathrm{d} x .
$$

Hence by the continuity of $I$ there exists $q_{\varepsilon} \in\left(\underline{q}_{\varepsilon}, \bar{q}_{\varepsilon}\right)$ for which 4.66 is satisfied.

We notice that $x_{\varepsilon}^{\prime \prime}-x_{\varepsilon}^{\prime}=2 \delta e^{-\delta / 4 \varepsilon}$ and it can be proved that the energy contribution due to the linear modification in $z_{\varepsilon}$ is of order $\delta e^{-\delta / 4 \varepsilon}$ too.

With an abuse of notation we now denote by $u_{\varepsilon}^{v}$ the restriction of $u_{\varepsilon}^{v}$ to the interval $(0,1)$; then by virtue of 4.64 ,

$$
\begin{aligned}
\lim _{\varepsilon \rightarrow 0} \mathcal{F}_{\varepsilon}^{k(2)}\left(u_{\varepsilon}^{v}\right)= & \lim _{\varepsilon \rightarrow 0}\left(-\left(C_{1}^{k}-C_{2}^{k}\right)^{2}\left(n_{1}^{v}+n_{2}^{v}\right) \delta+n_{1}^{v} \delta 2 C_{1}^{k}\left(\tanh \left(\frac{\delta}{4 \varepsilon}\right)-1\right) \frac{\delta}{\varepsilon}\right. \\
& \left.+n_{2}^{v} \delta 2 C_{2}^{k}\left(\tanh \left(\frac{\delta}{4 \varepsilon}\right)-1\right) \frac{\delta}{\varepsilon}+O\left(\delta e^{-\delta / 4 \varepsilon}\right)\right)\left[\frac{1}{\left(n_{1}^{v}+n_{2}^{v}+2\right) \delta}\right] \\
= & -\left(C_{1}^{k}-C_{2}^{k}\right)^{2} \frac{n_{1}^{v}+n_{2}^{v}}{n_{1}^{v}+n_{2}^{v}+2} .
\end{aligned}
$$

Since

$$
\lim _{\nu \rightarrow 0}-\left(C_{1}^{k}-C_{2}^{k}\right)^{2} \frac{n_{1}^{v}+n_{2}^{v}}{n_{1}^{v}+n_{2}^{v}+2}=-\left(C_{1}^{k}-C_{2}^{k}\right)^{2}
$$


a diagonalization argument yields a positive increasing function $v=v(\varepsilon)$ such that $v(\varepsilon) \rightarrow 0$ as $\varepsilon \rightarrow 0$ and

$$
\lim _{\varepsilon \rightarrow 0} \mathcal{F}_{\varepsilon}^{k(2)}\left(u_{\varepsilon}^{\nu(\varepsilon)}\right)=-\left(C_{1}^{k}-C_{2}^{k}\right)^{2} .
$$

Moreover, by using (4.64) it is easy to check that also $u_{\varepsilon}^{v(\varepsilon)} \rightarrow d$ in $L^{2}(0,1)$. Finally, starting from $u_{\varepsilon}^{\nu(\varepsilon)}$ a similar construction to that described in Remark 4.9 together with the assumption $\delta \ll \varepsilon^{1 / 2}$, yields a recovery sequence $\left(u_{\varepsilon}\right)$ also satisfying $\int_{0}^{1} u_{\varepsilon} \mathrm{d} x=d$, and hence the limsup inequality.

Theorem 4.10 shows that the second-order $\Gamma$-limit $\mathcal{F}^{k(2)}$ gives little information on the asymptotic behavior of minimizing sequences. Moreover, the next meaningful scaling is $\bar{\lambda}_{\infty}^{(3)}(\varepsilon)=\delta$ and takes into account only the energy contribution of a minimizing sequence at the boundary. Thus we may consider

$$
\mathcal{F}_{\varepsilon}^{k(3)}(u):=\frac{1}{\delta} \mathcal{F}_{\varepsilon}^{k(1)}(u)+\frac{\varepsilon}{\delta^{2}}\left(C_{1}^{k}-C_{2}^{k}\right)^{2} .
$$

The analysis of this energy and the one at the next scale will actually be a refinement of the one in the previous theorem, so that in the end Theorem 4.10 and Theorems 4.12 and 4.13 together give a description of the effect of oscillations at a scale $\delta$, which spans three different energetic scales.

THEOREM 4.12 Let $\delta$ be such that $\delta \ll \varepsilon^{1 / 2}$ and $1 / \delta \in \mathbb{N}$. The family of functionals $\mathcal{F}_{\varepsilon}^{k(3)}$ defined by (4.67) $\Gamma$-converges with respect to weak $L^{2}$-convergence to the functional defined on $L^{2}(0,1)$ by

$$
\mathcal{F}^{k(2)}(u)= \begin{cases}-\left(C_{1}^{k}+C_{2}^{k}\right) / 2 & \text { if } u \in L^{2}(0,1), 0 \leqslant u \leqslant 1 \text { a.e., and } \int_{0}^{1} u=d, \\ +\infty, & \text { otherwise. }\end{cases}
$$

Proof. The proof follows the one for the previous scale, the main difference being to modify the recovery sequence so that $u_{\varepsilon}(x)=1+k+s_{\varepsilon}$ on $(0, \delta / 4)$ and $u_{\varepsilon}(x)=1-k+s_{\varepsilon}$ on $(1-\delta / 4,1)$. Note that this is compatible with the construction in the proof of Theorem 4.10

The scale analysis performed in Theorem 4.10 suggests that the next meaningful scaling could be $e^{-\delta / 2 \varepsilon}$ as well as $\varepsilon e^{-\delta / 4 \varepsilon}$, as the higher-order energy contribution in terms of the scaled energy $\mathcal{F}_{\varepsilon}^{k(3)}$ is

Then, if

$$
\frac{1}{\varepsilon} e^{-\delta / 2 \varepsilon}+e^{-\delta / 4 \varepsilon}
$$

$$
\frac{1}{\varepsilon} e^{-\delta / 2 \varepsilon} \gg e^{-\delta / 4 \varepsilon}, \quad \text { i.e., } \quad e^{-\delta / 4 \varepsilon} \gg \varepsilon,
$$

we deduce that $\bar{\lambda}_{\infty}^{(4)}(\varepsilon)=e^{-\delta / 2 \varepsilon}$ and, as a consequence, the following $\Gamma$-convergence result for the scaled family

$$
\mathcal{F}_{\varepsilon}^{k(4)}(u):=\frac{\mathcal{F}_{\varepsilon}^{k(1)}(u)+\frac{\varepsilon}{\delta}\left(C_{1}^{k}-C_{2}^{k}\right)^{2}+\delta \frac{C_{1}^{k}+C_{2}^{k}}{2}}{e^{-\delta / 2 \varepsilon}} .
$$

THEOREM 4.13 Let $\varepsilon$ be such that $\varepsilon \ll e^{-\delta / 4 \varepsilon}$ and $1 / \delta \in \mathbb{N}$. The family of functionals $\mathcal{F}_{\varepsilon}^{k(4)}$ defined by (4.68) $\Gamma$-converges with respect to weak $L^{2}$-convergence to the functional defined on $L^{2}(0,1)$ by

$$
\mathcal{F}^{k(4)}(u)= \begin{cases}4\left(C_{2}^{k}-C_{1}^{k}\right) d-4 C_{2}^{k} & \text { if } u \in L^{2}(0,1), 0 \leqslant u \leqslant 1 \text { a.e., and } \int_{0}^{1} u=d, \\ +\infty & \text { otherwise. }\end{cases}
$$


Proof. The proof is a refinement of the computations in the proof of Theorem 4.10, with the boundary modifications hinted at in the proof of Theorem 4.12 We remark that at this scale we see the correction due to the difference between the values of the ordinates of the vertices of the two parabolas (4.63). Loosely speaking, this is the scale of the energy contributions due to the periodic optimal transitions with average $1+s_{\varepsilon}$ and with average $s_{\varepsilon}$, which, in the limit, give rise to

$$
\lim _{\varepsilon \rightarrow 0} \frac{2 C_{1}^{k}(\tanh (\delta / 4 \varepsilon)-1)}{e^{-\delta / 2 \varepsilon}}=-4 C_{1}^{k}, \quad \lim _{\varepsilon \rightarrow 0} \frac{2 C_{2}^{k}(\tanh (\delta / 4 \varepsilon)-1)}{e^{-\delta / 2 \varepsilon}}=-4 C_{2}^{k},
$$

respectively. Hence, we get the limit energy

$$
4\left(C_{2}^{k}-C_{1}^{k}\right) d-4 C_{2}^{k}
$$

with a recovery sequence that in order to preserve the integral constraint is a suitable combination of the two types of oscillations as above.

We notice that unfortunately the assumption $\varepsilon \ll e^{-\delta / 4 \varepsilon}$ together with $\delta \ll \varepsilon^{1 / 2}$ (see Theorem 4.10) is quite restrictive since it essentially reduces $\delta$ to be of type $\gamma \varepsilon|\log \varepsilon|$ with $0<\gamma<4$.

The last remark in this section is that actually $\bar{\lambda}_{\infty}^{(5)}(\varepsilon) \ll \varepsilon e^{-\delta / 4 \varepsilon}$ since a more accurate analysis shows that the choice of the linear function, joining the two different types of transitions in Theorem 4.10. Step 2, is not optimal and can be improved to obtain an energy contribution of higher order. Finally, if

$$
\mathcal{F}_{\varepsilon}^{k(5)}(u):=\frac{\mathcal{F}_{\varepsilon}^{k(1)}(u)+\frac{\varepsilon}{\delta}\left(C_{1}^{k}-C_{2}^{k}\right)^{2}+\delta \frac{C_{1}^{k}+C_{2}^{k}}{2}-e^{-\delta / 2 \varepsilon} 4\left(\left(C_{2}^{k}-C_{1}^{k}\right) d-4 C_{2}^{k}\right)}{\bar{\lambda}_{\infty}^{(5)}(\varepsilon)}
$$

we conjecture that $\mathcal{F}_{\varepsilon}^{k(5)} \stackrel{\Gamma}{\longrightarrow} \mathcal{F}^{k(5)}$ with

$$
\mathcal{F}^{k(5)}(u)= \begin{cases}C^{k} \#(S(u)) & \text { if } u \in B V((0,1) ;\{0,1\}), \text { and } \int_{0}^{1} u=d, \\ +\infty & \text { otherwise, }\end{cases}
$$

and $C^{k}$ a positive constant.

Summing up, in the case of large perturbations, by virtue of Theorems 4.2, 4.10, 4.12 and 4.13 we have established the following development by $\Gamma$-convergence:

$$
\begin{aligned}
F_{\varepsilon}^{k}(u) \stackrel{\Gamma}{=} & \int_{0}^{1} W^{* *}(u) \mathrm{d} x+\frac{\varepsilon}{\delta} \int_{0}^{1} \psi^{k}(u) \mathrm{d} x-\frac{\varepsilon^{2}}{\delta^{2}}\left(C_{1}^{k}-C_{2}^{k}\right)^{2}-\varepsilon \frac{C_{1}^{k}+C_{2}^{k}}{2} \\
& +\frac{\varepsilon}{\delta} e^{-\delta / 2 \varepsilon}\left(4\left(C_{2}^{k}-C_{1}^{k}\right) d-4 C_{2}^{k}\right)+o\left(\frac{\varepsilon^{2}}{\delta} e^{-\delta / 4 \varepsilon}\right)
\end{aligned}
$$

(with restrictions on $\varepsilon$ and $\delta$ for the higher-order terms).

We conclude by noticing that (4.46) and (4.69) turn out to coincide for $k=1 / 2$.

\section{5. $\delta \ll \varepsilon$ : the case of fast oscillations}

In this section we treat the case when the scale of oscillation $\delta$ is much smaller than the scale of the transition layer $\varepsilon$. In particular, we show that in this case, up to choosing $\delta$ sufficiently small, 
the presence of small-scale heterogeneities does not essentially affect the $\Gamma$-convergence process at first order either.

We recall that for $k \leqslant 1 / 2$ Theorem 3.1 asserts that $F_{\varepsilon}^{k} \stackrel{\Gamma}{\longrightarrow} F_{0}^{k(0)}$ with $F_{0}^{k(0)}(u)=\int_{0}^{1} W_{0}^{k}(u) \mathrm{d} x$ and $\min F_{0}^{k(0)}=k^{2}=F_{0}^{k(0)}(u)$ for every $u \in L^{2}(0,1)$ with $|u| \leqslant 1$ a.e. Thus we are now interested in determining the scaling $\lambda_{0}^{(1)}(\varepsilon)$, to study the asymptotic behavior of the family of scaled functionals

$$
I_{\varepsilon}^{k(1)}(u):=\frac{F_{\varepsilon}^{k}(u)-k^{2}}{\lambda_{0}^{(1)}(\varepsilon)} .
$$

To this end, we perform a first heuristic scale analysis. For simplicity we assume that $1 / \delta \in \mathbb{N}$. Then we notice that, for instance, $\bar{v}_{\varepsilon}=1$ is a minimizing sequence for $\left(F_{\varepsilon}^{k}\right)$ as $F_{\varepsilon}^{k}\left(\bar{v}_{\varepsilon}\right)=k^{2}$. Nevertheless, we want to show that for any (small) fixed $\varepsilon>0, \bar{v}_{\varepsilon}$ is not an absolute minimizer for $F_{\varepsilon}^{k}$. In fact,

$$
\begin{aligned}
\min F_{\varepsilon}^{k} \leqslant & \min \left\{F_{\varepsilon}^{k}: u(0)=u(1)=1\right\} \\
\leqslant & \min \left\{\frac{1}{\delta} \int_{0}^{\delta}\left(W^{k}\left(\frac{x}{\delta}, u\right)+\varepsilon^{2}\left(u^{\prime}\right)^{2}\right) \mathrm{d} x: u(0)=u(\delta)=1\right\} \\
\leqslant & \min \left\{\frac{1}{\delta} \int_{0}^{\delta / 2}\left((u-1-k)^{2}+\varepsilon^{2}\left(u^{\prime}\right)^{2}\right) \mathrm{d} x\right. \\
& \left.+\frac{1}{\delta} \int_{0}^{\delta / 2}\left((u-1+k)^{2}+\varepsilon^{2}\left(u^{\prime}\right)^{2}\right) \mathrm{d} x: u(0)=u(\delta)=1\right\} \\
\leqslant & \min \left\{\frac{2}{\delta} \int_{0}^{\delta / 2}\left((u-1-k)^{2}+\varepsilon^{2}\left(u^{\prime}\right)^{2}\right) \mathrm{d} x: u(0)=u\left(\frac{\delta}{2}\right)=1\right\} \\
= & 4 k^{2} \frac{\varepsilon}{\delta} \tanh \left(\frac{\delta}{4 \varepsilon}\right)=k^{2}-\frac{k^{2}}{48} \frac{\delta^{2}}{\varepsilon^{2}}+\frac{k^{2}}{1920} \frac{\delta^{4}}{\varepsilon^{4}}+O\left(\frac{\delta^{6}}{\varepsilon^{6}}\right) \text { as } \varepsilon \rightarrow 0,
\end{aligned}
$$

and the minimum 5.1 is attained at

$$
v(x):=1+k-k \cosh \left(\frac{\delta-4 x}{4 \varepsilon}\right)\left(\cosh \left(\frac{\delta}{4 \varepsilon}\right)\right)^{-1} .
$$

Hence the previous computations show that it is more energetically convenient to "oscillate around 1" than to be identically 1 . Clearly, the same conclusion still applies to the constant phase -1 . Thus a minimizing sequence may well be the result of a combination (on a suitable scale) of oscillations around 1 with oscillations around -1 . Finally, as the presence of the singular perturbation in the gradient introduces $\varepsilon$ as the length for the layer of a transition between the two "oscillating phases" \pm 1 , we deduce that the contribution of a minimizing sequence in terms of the energy $F_{\varepsilon}^{k}-k^{2}$ is (at least) of order

$$
\varepsilon+\frac{\delta^{2}}{\varepsilon^{2}}
$$

This section will be entirely devoted to the case $\delta \ll \varepsilon^{3 / 2}$, which yields

$$
\lambda_{0}^{(1)}(\varepsilon)=\varepsilon,
$$

since in view of (5.2) we expect to obtain constant $\Gamma$-limits for other choices of the scaling $\lambda_{0}^{(1)}$. 
We remark that also the asymptotic analysis for the "critical case" $\delta \simeq \varepsilon^{3 / 2}$ (or more generally, $\delta \simeq \varepsilon^{(2 n+1) / 2 n}$ for some $n \in \mathbb{N}$ ) yields a $\Gamma$-limit of Modica-Mortola type. Nonetheless, it seems that in this case the two phenomena of oscillations and phase transition may interact in a nontrivial way, thus introducing some additional issues that will not be developed here.

THEOREM 5.1 Let $k<1 / 2$ and let $\delta$ be such that

$$
\delta \ll \varepsilon^{3 / 2} .
$$

Then the functionals $I_{\varepsilon}^{k}$ defined on $L^{2}(0,1)$ by

$$
I_{\varepsilon}^{k}(u):= \begin{cases}\int_{0}^{1}\left(\frac{1}{\varepsilon}\left(W^{k}\left(\frac{x}{\delta}, u\right)-k^{2}\right)+\varepsilon\left(u^{\prime}\right)^{2}\right) \mathrm{d} x & \text { if } u \in W^{1,2}(0,1), \\ +\infty, & \text { otherwise }\end{cases}
$$

$\Gamma$-converge with respect to strong $L^{2}$-convergence to the functional

$$
I^{k}(u)= \begin{cases}C_{\left(\bar{W}^{k}-k^{2}\right)} \#(S(u)) & \text { if } u \in B V((0,1) ;\{ \pm 1\}), \\ +\infty & \text { otherwise, }\end{cases}
$$

with $\bar{W}^{k}$ as in 3.5 and

$$
C_{\left(\bar{W}^{k}-k^{2}\right)}:=2 \int_{-1}^{1} \sqrt{\bar{W}^{k}(s)-k^{2}} \mathrm{~d} s=2(1-k)^{2}+(2 k-1) \ln (1-2 k) .
$$

Proof. Step 1: $\Gamma$-liminf inequality. Let $u_{\varepsilon} \rightarrow u$ in $L^{2}(0,1)$ be $\operatorname{such}$ that $\sup _{\varepsilon} I_{\varepsilon}^{k}\left(u_{\varepsilon}\right)<+\infty$; for fixed $\varepsilon>0$ define the set $J^{\delta}$ and, on $J^{\delta}$, the function $v_{\varepsilon}$ by

$$
J^{\delta}:=\bigcup_{i=1}^{[1 / \delta]}((i-1) \delta, i \delta), \quad v_{\varepsilon}(x):=\sum_{i=1}^{[1 / \delta]} u_{\varepsilon}^{i} \chi_{((i-1) \delta, i \delta)}(x)
$$

with

$$
u_{\varepsilon}^{i}:=f_{(i-1) \delta}^{i \delta} u_{\varepsilon} \mathrm{d} t \quad \text { for } i=1, \ldots,[1 / \delta] .
$$

By the Jensen Inequality it is immediate to check that

$$
\left\|v_{\varepsilon}\right\|_{L^{2}\left(J^{\delta}\right)} \leqslant\left\|u_{\varepsilon}\right\|_{L^{2}\left(J^{\delta}\right)},
$$

while from the Poincaré Inequality and its scaling properties we have

$$
\left\|u_{\varepsilon}-v_{\varepsilon}\right\|_{L^{2}\left(J^{\delta}\right)} \leqslant \delta\left\|u_{\varepsilon}^{\prime}\right\|_{L^{2}\left(J^{\delta}\right)} .
$$

A first estimate gives

$$
I_{\varepsilon}^{k}\left(u_{\varepsilon}\right) \geqslant \int_{J^{\delta}}\left(\frac{1}{\varepsilon}\left(W^{k}\left(\frac{x}{\delta}, u_{\varepsilon}\right)-k^{2}\right)+\varepsilon\left(u_{\varepsilon}^{\prime}\right)^{2}\right) \mathrm{d} x-\frac{k^{2}}{\varepsilon} \int_{\delta[1 / \delta]}^{1} \mathrm{~d} x,
$$


hence

$$
\liminf _{\varepsilon \rightarrow 0} I_{\varepsilon}^{k}\left(u_{\varepsilon}\right) \geqslant \liminf _{\varepsilon \rightarrow 0} \int_{J^{\delta}}\left(\frac{1}{\varepsilon}\left(W^{k}\left(\frac{x}{\delta}, u_{\varepsilon}\right)-k^{2}\right)+\varepsilon\left(u_{\varepsilon}^{\prime}\right)^{2}\right) \mathrm{d} x .
$$

We now claim that

$$
\lim _{\varepsilon \rightarrow 0} \frac{1}{\varepsilon} \int_{J^{\delta}}\left(W^{k}\left(\frac{x}{\delta}, u_{\varepsilon}\right)-\bar{W}^{k}\left(u_{\varepsilon}\right)\right) \mathrm{d} x=0 .
$$

To prove this claim we first remark that $W^{k}(y, \cdot)$ has the following local Lipschitz property:

$$
\left|W^{k}\left(y, s_{1}\right)-W^{k}\left(y, s_{2}\right)\right| \leqslant\left(2(k+1)+\left|s_{1}\right|+\left|s_{2}\right|\right)\left|s_{1}-s_{2}\right| \quad \text { for a.e. } y \in \mathbb{R} \text { and } s_{1}, s_{2} \in \mathbb{R} \text {. }
$$

By a simple averaging over $(0,1)$ it immediately follows that 5.7 is also satisfied by $\bar{W}^{k}$. Moreover, by the definition of $v_{\varepsilon}$ and the 1-periodicity of $W^{k}(\cdot, s)$,

$$
\begin{aligned}
\int_{J^{\delta}} W^{k}\left(\frac{x}{\delta}, u_{\varepsilon}\right) \mathrm{d} x & =\sum_{i=1}^{[1 / \delta]} \int_{(i-1) \delta}^{i \delta} W^{k}\left(\frac{x}{\delta}, u_{\varepsilon}^{i}\right) \mathrm{d} x=\sum_{i=1}^{[1 / \delta]} \int_{0}^{\delta} W^{k}\left(\frac{x}{\delta}, u_{\varepsilon}^{i}\right) \mathrm{d} x \\
& =\sum_{i=1}^{[1 / \delta]} \delta \int_{0}^{1} W^{k}\left(x, u_{\varepsilon}^{i}\right) \mathrm{d} x=\sum_{i=1}^{[1 / \delta]} \delta \bar{W}^{k}\left(u_{\varepsilon}^{i}\right)=\int_{J^{\delta}} \bar{W}^{k}\left(v_{\varepsilon}\right) \mathrm{d} x
\end{aligned}
$$

Then, by adding and subtracting $1 / \varepsilon \int_{J^{\delta}} W^{k}\left(\frac{x}{\delta}, v_{\varepsilon}\right) \mathrm{d} x$ in 5.6 , by virtue of 5.7) and the local Lipschitz continuity of $\bar{W}^{k}$ we have

$$
\begin{aligned}
& \frac{1}{\varepsilon}\left|\int_{J^{\delta}}\left(W^{k}\left(\frac{x}{\delta}, u_{\varepsilon}\right)-\bar{W}^{k}\left(u_{\varepsilon}\right)\right) \mathrm{d} x\right| \\
& \quad \leqslant \frac{1}{\varepsilon} \int_{J^{\delta}}\left|W^{k}\left(\frac{x}{\delta}, u_{\varepsilon}\right)-W^{k}\left(\frac{x}{\delta}, v_{\varepsilon}\right)\right| \mathrm{d} x+\frac{1}{\varepsilon} \int_{J^{\delta}}\left|\bar{W}^{k}\left(u_{\varepsilon}\right)-\bar{W}^{k}\left(v_{\varepsilon}\right)\right| \mathrm{d} x \\
& \leqslant \frac{2}{\varepsilon} \int_{J^{\delta}}\left(2(k+1)+\left|u_{\varepsilon}\right|+\left|v_{\varepsilon}\right|\right)\left|u_{\varepsilon}-v_{\varepsilon}\right| \mathrm{d} x \\
& \leqslant \frac{1}{\varepsilon} C\left(1+\left\|u_{\varepsilon}\right\|_{L^{2}\left(J^{\delta}\right)}+\left\|v_{\varepsilon}\right\|_{L^{2}\left(J^{\delta}\right)}\right)\left\|u_{\varepsilon}-v_{\varepsilon}\right\|_{L^{2}\left(J^{\delta}\right)} \leqslant C \frac{\delta}{\varepsilon}\left\|u_{\varepsilon}^{\prime}\right\|_{L^{2}(0,1)},
\end{aligned}
$$

where we have used 5.4 and 5.5 in the last inequality. Recalling that $\sup _{\varepsilon} I_{\varepsilon}^{k}\left(u_{\varepsilon}\right)<+\infty$ in particular implies

$$
\left\|u_{\varepsilon}^{\prime}\right\|_{L^{2}(0,1)} \leqslant \frac{C}{\varepsilon^{1 / 2}},
$$

and by combining (5.8), 5.9) and invoking hypothesis 5.3 we get the claim.

As a consequence,

$$
\liminf _{\varepsilon \rightarrow 0} I_{\varepsilon}^{k}\left(u_{\varepsilon}\right) \geqslant \liminf _{\varepsilon \rightarrow 0} \int_{0}^{\delta[1 / \delta]}\left(\frac{1}{\varepsilon}\left(\bar{W}^{k}\left(u_{\varepsilon}\right)-k^{2}\right)+\varepsilon\left(u_{\varepsilon}^{\prime}\right)^{2}\right) \mathrm{d} x,
$$

so that we reduce to the case of a sequence of functionals with a homogeneous, double-well potential, with wells at \pm 1 . Moreover, up to a slight modification of the proof of the ModicaMortola compactness result, 5.10) implies that if $\left(u_{\varepsilon}\right)$ is $\operatorname{such}$ that $\sup _{\varepsilon} I_{\varepsilon}^{k}\left(u_{\varepsilon}\right)<+\infty$, then 
$u_{\varepsilon} \rightarrow u$ in $L^{2}(0,1)$, with $u \in B V((0,1) ;\{ \pm 1\})$. Finally, a direct application of the Modica-Mortola Theorem yields

$$
\begin{aligned}
\liminf _{\varepsilon \rightarrow 0} I_{\varepsilon}^{k}\left(u_{\varepsilon}\right) & \geqslant \liminf _{\varepsilon \rightarrow 0} \int_{0}^{a}\left(\frac{1}{\varepsilon}\left(\bar{W}^{k}\left(u_{\varepsilon}\right)-k^{2}\right)+\varepsilon\left(u_{\varepsilon}^{\prime}\right)^{2}\right) \mathrm{d} x \\
& \geqslant\left(2 \int_{-1}^{1} \sqrt{\bar{W}^{k}(s)-k^{2}} \mathrm{~d} s\right) \#(S(u) \cap(0, a))
\end{aligned}
$$

for any fixed $a \in(0,1)$. Then, passing to the sup over $a \in(0,1)$, we get the $\Gamma$-liminf inequality.

Step 2: $\Gamma$-limsup inequality. We have to construct a recovery sequence for $u \in B V(0,1)$ with $u \in\{ \pm 1\}$ a.e.; it will suffice to approximate

$$
u(x)= \begin{cases}-1 & \text { if } x<x_{0} \\ 1 & \text { if } x \geqslant x_{0}\end{cases}
$$

with $x_{0} \in(0,1)$.

We show that the limsup inequality easily follows from the limsup inequality for the functionals

$$
\int_{0}^{1}\left(\frac{1}{\varepsilon}\left(\bar{W}^{k}(u)-k^{2}\right)+\varepsilon\left(u^{\prime}\right)^{2}\right) \mathrm{d} x .
$$

To this end, arguing as in the Modica-Mortola construction, for any fixed $\eta>0$ we can find a number $T>0$ and a function $v \in W^{1,2}(-T, T)$ such that $v(-T)=-1, v(T)=1$ and

$$
\int_{-T}^{T}\left(\bar{W}^{k}(v)-k^{2}+\left(v^{\prime}\right)^{2}\right) \mathrm{d} x \leqslant 2 \int_{-1}^{1} \sqrt{\bar{W}^{k}(s)-k^{2}} \mathrm{~d} s+\eta .
$$

Then, recalling that $\delta \ll \varepsilon$, we see that a recovery sequence for $5.11-(5.12)$ is given by

$$
u_{\varepsilon}(x)= \begin{cases}-1 & \text { if } x<x_{0}^{\delta}-\varepsilon T, \\ v\left(\left(x-x_{0}^{\delta}\right) / \varepsilon\right) & \text { if } x_{0}^{\delta}-\varepsilon T \leqslant x \leqslant x_{0}^{\delta}+\varepsilon T, \\ 1 & \text { if } x>x_{0}^{\delta}+\varepsilon T,\end{cases}
$$

with $x_{0}^{\delta}=\left[x_{0} / \delta\right] \delta$. We claim that $\left(u_{\varepsilon}\right)$ is also a recovery sequence for $I_{\varepsilon}^{k}$. First we find

$$
I_{\varepsilon}^{k}\left(u_{\varepsilon}\right)=\int_{x_{0}^{\delta}-\varepsilon T}^{x_{0}^{\delta}+\varepsilon T}\left(\frac{1}{\varepsilon}\left(W^{k}\left(\frac{x}{\delta}, u_{\varepsilon}\right)-k^{2}\right)+\varepsilon\left(u_{\varepsilon}^{\prime}\right)^{2}\right) \mathrm{d} x=\int_{-T}^{T}\left(W^{k}\left(\frac{\varepsilon}{\delta} x, v\right)-k^{2}+\left(v^{\prime}\right)^{2}\right) \mathrm{d} x
$$

The next step is to prove that

$$
\lim _{\varepsilon \rightarrow 0} \int_{-T}^{T} W^{k}\left(\frac{\varepsilon}{\delta} x, v\right) \mathrm{d} x=\int_{-T}^{T} \bar{W}^{k}(v) \mathrm{d} x .
$$

Setting

$$
W_{\varepsilon}^{k}(x):=W^{k}\left(\frac{\varepsilon}{\delta} x, v\right) \quad \text { for a.e. } x \in(-T, T),
$$


we have

$$
0 \leqslant W_{\varepsilon}^{k} \leqslant 2\left((k+1)^{2}+|v|^{2}\right) \quad \text { a.e. in }(-T, T),
$$

from which we deduce $\left\|W_{\varepsilon}^{k}\right\|_{L^{1}(-T, T)} \leqslant C$ and that $\left(W_{\varepsilon}^{k}\right)$ is equi-integrable on $(-T, T)$. Then by applying the Dunford-Pettis Criterion, upon passing to a subsequence (not relabeled) we have

$$
W_{\varepsilon}^{k} \rightarrow f \quad \text { in } L^{1}(-T, T),
$$

while by the Lebesgue Theorem,

$$
f(x)=\lim _{r \rightarrow 0^{+}} \int_{x-r}^{x+r} f(y) \mathrm{d} y \quad \text { for a.e. } x \in(-T, T) .
$$

Moreover, 5.15) implies in particular that, for $x \in(-T, T)$ and for sufficiently small $r>0$,

$$
\lim _{\varepsilon \rightarrow 0} \int_{x-r}^{x+r} W_{\varepsilon}^{k}(y) \mathrm{d} y=\int_{x-r}^{x+r} f(y) \mathrm{d} y,
$$

and consequently

$$
\lim _{r \rightarrow 0^{+}} \lim _{\varepsilon \rightarrow 0} \int_{x-r}^{x+r} W_{\varepsilon}^{k}(y) \mathrm{d} y=f(x) \quad \text { for a.e. } x \in(-T, T) .
$$

On the other hand,

$$
\begin{aligned}
\int_{x-r}^{x+r} W_{\varepsilon}^{k}(y) \mathrm{d} y= & \int_{x-r}^{x+r} W^{k}\left(\frac{\varepsilon}{\delta} y, v\right) \mathrm{d} y-f_{x-r}^{x+r} W^{k}\left(\frac{\varepsilon}{\delta} y, v(x)\right) \mathrm{d} y \\
& +\int_{x-r}^{x+r} W^{k}\left(\frac{\varepsilon}{\delta} y, v(x)\right) \mathrm{d} y
\end{aligned}
$$

with

$$
\left|f_{x-r}^{x+r}\left(W^{k}\left(\frac{\varepsilon}{\delta} y, v\right)-W^{k}\left(\frac{\varepsilon}{\delta} y, v(x)\right)\right) \mathrm{d} y\right| \leqslant f_{x-r}^{x+r}(2(k+1)+|v(x)|+|v|)|v-v(x)| \mathrm{d} y
$$

and

$$
\lim _{\varepsilon \rightarrow 0} \int_{x-r}^{x+r} W^{k}\left(\frac{\varepsilon}{\delta} y, v(x)\right) \mathrm{d} y=\int_{x-r}^{x+r} \bar{W}^{k}(v(x)) \mathrm{d} y=\bar{W}^{k}(v(x)) .
$$

Letting in 5.16) first $\varepsilon \rightarrow 0$, then $r \rightarrow 0$, we obtain $f(x)=\bar{W}^{k}(v(x))$ for a.e. $x \in(-T, T)$; hence, from 5.15) we get 5.14. Finally, combining 5.14 and 5.13 gives

$$
\limsup _{\varepsilon \rightarrow 0} I_{\varepsilon}^{k}\left(u_{\varepsilon}\right) \leqslant 2 \int_{-1}^{1} \sqrt{\bar{W}^{k}(s)-k^{2}} \mathrm{~d} s+\eta=I^{k}(u)+\eta,
$$

and the conclusion follows by the arbitrariness of $\eta$.

REMARK 5.2 The above theorem states that formally we may first perform the homogenization procedure for fixed $\varepsilon$, by letting $\delta \rightarrow 0$, and then apply the Modica-Mortola Theorem to the functionals

$$
\int_{0}^{1}\left(\bar{W}^{k}(u)-k^{2}+\varepsilon^{2}\left(u^{\prime}\right)^{2}\right) \mathrm{d} x .
$$


Since just as for the Modica-Mortola functionals, the equi-coercivity at scale $\varepsilon$ improves to strong- $L^{2}$ equi-coercivity, we may (a posteriori) also compute the zero-order $\Gamma$-limit with respect to strong $L^{2}$-convergence, obtaining

$$
F_{0}^{k(0)}(u)=\int_{0}^{1} \bar{W}^{k}(u) \mathrm{d} x
$$

Thus, for $\delta \ll \varepsilon^{3 / 2}$ and $k<1 / 2$ we find that a $\Gamma$-development for $F_{\varepsilon}^{k}$ with respect to weak $L^{2}$-convergence is given by

$$
F_{\varepsilon}^{k}(u)=\int_{0}^{1}\left(\bar{W}^{k}\right)^{* *}(u) \mathrm{d} x+\varepsilon C_{\left(\bar{W}^{k}-k^{2}\right)} \#(S(u))+O\left(\frac{\delta^{2}}{\varepsilon^{2}}\right),
$$

while a $\Gamma$-development with respect to strong $L^{2}$-convergence is

$$
F_{\varepsilon}^{k}(u)=\int_{0}^{1} \bar{W}^{k}(u) \mathrm{d} x+\varepsilon C_{\left(\bar{W}^{k}-k^{2}\right)} \#(S(u))+O\left(\frac{\delta^{2}}{\varepsilon^{2}}\right) .
$$

The last part of this section is devoted to the case $k>1 / 2$. In this regime, for the zero-order $\Gamma$ limit we have $\min F_{0}^{k(0)}=(1-k)^{2}$ and the minimum is attained at $u=0$ (see Fig. 8). Nevertheless, since the effective potential $W_{0}^{k}$ is not strictly convex, we may proceed as in Section 4.3.2 Thus, setting

$$
\tau^{k}(s):=(2 k-1) s-k+3 / 4
$$

we can consider, for instance, the family of functionals

$$
F_{\varepsilon}^{k}(u)-\int_{0}^{1} \tau^{k}(u) \mathrm{d} x,
$$

which, under the assumption

$$
\int_{0}^{1} u \mathrm{~d} x=d \in(k-1 / 2, k+1 / 2),
$$

only differs from $F_{\varepsilon}^{k}$ by a constant.

Now it is immediate to prove that the $\Gamma$-convergence result stated in Theorem 3.1 preserves the integral constraint (5.20) and hence that 5.19$) \Gamma$-converges to the functional

$$
\int_{0}^{1}\left(W_{0}^{k}(u)-\tau^{k}(u)\right) \mathrm{d} x, \quad u \in L^{2}(0,1), \int_{0}^{1} u \mathrm{~d} x=d,
$$

which vanishes at any function $u \in L^{2}(0,1)$ with $|u-k| \leqslant 1 / 2$ a.e. and such that $\int_{0}^{1} u \mathrm{~d} x=d$. Moreover, a similar scale analysis to that performed for $k \leqslant 1 / 2$ also applies in this case, leading to the following result.

THEOREM 5.3 Let $k>1 / 2$ and choose $\delta$ satisfying 5.3 . Then the functionals $\mathcal{I}_{\varepsilon}^{k}$ defined on $L^{2}(0,1)$ by

$$
\mathcal{I}_{\varepsilon}^{k}(u):= \begin{cases}\int_{0}^{1}\left(\frac{1}{\varepsilon}\left(W^{k}\left(\frac{x}{\delta}, u\right)-\tau^{k}(u)\right)+\varepsilon\left(u^{\prime}\right)^{2}\right) \mathrm{d} x & \text { if } u \in W^{1,2}(0,1) \text { and } \int_{0}^{1} u=d, \\ +\infty & \text { otherwise, }\end{cases}
$$


$\Gamma$-converge with respect to strong $L^{2}$-convergence to the functional

$$
\mathcal{I}^{k}(u)= \begin{cases}C_{\left(\bar{W}^{k}-\tau^{k}\right)} \#(S(u)) & \text { if } u \in B V((0,1) ;\{k \pm 1 / 2\}) \text { and } \int_{0}^{1} u=d, \\ +\infty & \text { otherwise, }\end{cases}
$$

where $C_{\left(\bar{W}^{k}-\tau^{k}\right)}:=2 \int_{k-1 / 2}^{k+1 / 2} \sqrt{\bar{W}^{k}(s)-\tau^{k}(s)} \mathrm{d} s=1 / 2$.

Proof. The proof exactly follows the lines of that for $k<1 / 2$, while a recovery sequence satisfying $(5.20)$ can be obtained by a carefully chosen translation of a recovery sequence for the nonconstrained problem (see, e.g., [3, Theorem 6.7]).

To conclude this section we notice that the energy contribution of an optimal transition is continuous in $k$, i.e.,

$$
\lim _{k \rightarrow \frac{1}{2}^{-}} C_{\left(\bar{W}^{k}-k^{2}\right)}=\lim _{k \rightarrow \frac{1}{2}^{+}} C_{\left(\bar{W}^{k}-\tau^{k}\right)}=\frac{1}{2},
$$

hence the result for $k=1 / 2$ is described by both Theorem 5.1 and Theorem 5.3

\section{6. $\delta \sim \varepsilon$ : the critical case}

In this last section we briefly outline the case when the scale of oscillation $\delta$ is of the same order as the scale of the transition layer $\varepsilon$. In this case the zero-order $\Gamma$-limit's energy density $W_{\ell}^{k}$ is described by the asymptotic formula $(3.3)$. By optimizing the procedure leading to $\sqrt{5.2}$, we wet

$$
W_{\ell}^{k}(s)= \begin{cases}\frac{2 C_{1}^{k}}{\ell} \tanh \left(\frac{\ell}{4}\right), & |s| \leqslant 1, \\ (|s|-1)^{2}+\frac{2 C_{1}^{k}}{\ell} \tanh \left(\frac{\ell}{4}\right), & |s|>1,\end{cases}
$$

for $k \leqslant 1 / 2$, while

$$
W_{\ell}^{k}(s)=\left\{\begin{array}{lr}
s^{2}+\frac{2 C_{2}^{k}}{\ell} \tanh \left(\frac{\ell}{4}\right), & |s|<\left(k-\frac{1}{2}\right) \frac{4}{\ell} \tanh \left(\frac{\ell}{4}\right), \\
(2 k-1) \frac{4}{\ell} \tanh \left(\frac{\ell}{4}\right)|s|+\left((1-k)^{2}-\frac{(2 k-1)^{2}}{\ell} \tanh \left(\frac{\ell}{4}\right)\right) \frac{4}{\ell} \tanh \left(\frac{\ell}{4}\right), & \left(k-\frac{1}{2}\right) \frac{4}{\ell} \tanh \left(\frac{\ell}{4}\right) \leqslant|s| \leqslant\left(k-\frac{1}{2}\right) \frac{4}{\ell} \tanh \left(\frac{\ell}{4}\right)+1, \\
(|s|-1)^{2}+\frac{2 C_{1}^{k}}{\ell} \tanh \left(\frac{\ell}{4}\right), & |s|>\left(k-\frac{1}{2}\right) \frac{4}{\ell} \tanh \left(\frac{\ell}{4}\right)+1,
\end{array}\right.
$$

for $k>1 / 2$. Note that these potentials have the same form of the potentials $W_{0}^{k}$ for $k \leqslant 1 / 2$ and for $k>1 / 2$ (see Fig. 8). Moreover, recalling that $C_{1}^{k}=2 k^{2}, C_{2}^{k}=2(k-1)^{2}$ and 3.6 - -3.8 it is immediate to check that for every fixed $k \in(0,1)$ the effective potential $W_{\ell}^{k}$ is continuous with respect to $\ell$ in the extreme regimes; i.e., $W_{\ell}^{k} \rightarrow W_{\infty}^{k}$ as $\ell \rightarrow \infty$ and $W_{\ell}^{k} \rightarrow W_{0}^{k}$ as $\ell \rightarrow 0$.

The behavior of minimizing sequences in the case $k \leqslant 1 / 2$ can be pictured as in Fig. 77, where the oscillations are not dumped as $\varepsilon \rightarrow 0$. The same picture also holds in the case $k>1 / 2$ but 
now the oscillations are "around" $(k-1 / 2)(4 / \ell) \tanh (\ell / 4)$ and $(k-1 / 2)(4 / \ell) \tanh (\ell / 4)+1$ if a positive volume constraint is added. In both cases oscillations of the ground states do not reach the minimum values for $W^{k}$, thus preventing the degenerate situation of the case $k>1 / 2$ and $\ell=+\infty$ (see Fig. 6), and hence the subsequent effects are at scale $\varepsilon$ and are due to phase transitions and boundary layers. Note that the bulk contribution due to oscillations is now at scale 1 and is included in the definition of $W_{\ell}^{k}$. We state the first-order $\Gamma$-convergence result for the case $k<1 / 2$ only, the other one being analogous upon addition of an integral constraint in the spirit of the previous analyses of Section 4.3.2 and Section 5 .

It can be proved that there exists a unique 1-periodic function $v_{\ell}^{+}$(the ground state) minimizing the problem, giving $W_{\ell}^{k}(1)$ with average 1 and $\max v_{\ell}^{+}-\min v_{\ell}^{+}<2$. Note that by the symmetries of the problem, $v_{\ell}^{-}:=v_{\ell}^{+}-2$ is a minimizer for $W_{\ell}^{k}(-1)$.

The surface tension for the first-order $\Gamma$-limit is then defined by

$$
\begin{aligned}
C_{\ell}^{k, s}=\inf _{n \in \mathbb{N}} \inf \left\{\frac{1}{2 n} \int_{-n}^{n}\left(W^{k}(y, v)+\frac{1}{\ell^{2}}\left(v^{\prime}\right)^{2}\right) \mathrm{d} y\right. & -\frac{2 C_{1}^{k}}{\ell} \tanh \left(\frac{\ell}{4}\right): \\
& \left.v \in W^{1,2}(-n, n), v( \pm n)=v_{\ell}^{ \pm}( \pm n)\right\},
\end{aligned}
$$

and the corresponding boundary-layer energy by

$$
\begin{aligned}
C_{\ell}^{k, b}=\inf _{n \in \mathbb{N}} \inf \left\{\frac{1}{n} \int_{0}^{n}\left(W^{k}(y, v)+\frac{1}{\ell^{2}}\left(v^{\prime}\right)^{2}\right) \mathrm{d} y-\frac{2 C_{1}^{k}}{\ell} \tanh \left(\frac{\ell}{4}\right):\right. & \\
v & \left.\in W^{1,2}(0, n), v(n)=v_{\ell}^{+}(n)\right\} .
\end{aligned}
$$

The first-order $\Gamma$-limit can then be stated as follows.

TheOREM 6.1 Let $k<1 / 2$ and $1 / \delta \in \mathbb{N}$. Then the functionals $H_{\varepsilon}^{k}$ defined on $L^{2}(0,1)$ by

$$
H_{\varepsilon}^{k}(u):= \begin{cases}\int_{0}^{1}\left(\frac{1}{\varepsilon}\left(W^{k}\left(\frac{x}{\delta}, u\right)-\frac{2 C_{1}^{k}}{\ell} \tanh \left(\frac{\ell}{4}\right)\right)+\varepsilon\left(u^{\prime}\right)^{2}\right) \mathrm{d} x & \text { if } u \in W^{1,2}(0,1), \\ +\infty & \text { otherwise, }\end{cases}
$$

$\Gamma$-converge with respect to weak $L^{2}$-convergence to the functional

$$
H^{k}(u)= \begin{cases}C_{\ell}^{k, s} \#(S(u))-2 C_{\ell}^{k, b} & \text { if } u \in B V((0,1) ;\{ \pm 1\}), \\ +\infty & \text { otherwise. }\end{cases}
$$

We omit the proof, which follows the one for the case $\ell=0$.

\section{Acknowledgments}

The second author thanks the Dipartimento di Matematica "G. Castelnuovo", Università di Roma "La Sapienza", where most of this work was carried out and supported during her Ph.D. program. 


\section{REFERENCES}

1. Alberti, G. Variational models for phase transitions, an approach via $\Gamma$-convergence. Calculus of Variations and Partial Differential Equations, L. Ambrosio and N. Dancer (eds.), Springer, Berlin (2000), 95-114. Zbl 0957.35017 MR 1757697

2. Anzellotti, G., \& Baldo, S. Asymptotic development by $\Gamma$-convergence. Appl. Math. Optim. 27 (1993), 105-123. Zbl 0780.49011 MR 1202527

3. BRAides, A. $\Gamma$-convergence for Beginners. Oxford Univ. Press, Oxford (2002). Zbl pre01865939 MR 1968440

4. Braides, A., \& Truskinovsky, L. Asymptotic expansions by $\Gamma$-convergence, Contin. Mech. Thermodyn. 20 (2008), 21-62. MR 2398821

5. Dirr, N., Lucia, M., \& Novaga, M. $\Gamma$-convergence of the Allen-Cahn energy with an oscillating forcing term. Interfaces Free Bound. 8 (2006), 47-78. Zbl 1106.49053 MR 2231252

6. Dirr, N., Lucia, M., \& Novaga, M. Gradient theory of phase transitions with a rapidly oscillating forcing term. Asymptot. Anal. 60 (2008), 29-59. Zbl pre05504511 MR 2463798

7. Francfort, G. A., \& MÜller, S. Combined effects of homogenization and singular perturbation in elasticity. J. Reine Angew. Math. 454 (1994), 1-35. Zbl 0802.35007| MR 1288677

8. ModicA, L. The gradient theory of phase transitions and the minimal interface criterion. Arch. Ration. Mech. Anal. 98 (1987), 123-142. Zbl 0616.76004 MR 0866718

9. Modica, L., \& Mortola, S. Un esempio di $\Gamma$-convergenza. Boll. Un. Mat. Ital. B 14 (1977), 285-299. Zbl 0356.49008 MR 0445362 